\title{
Hopf bifurcation for the hydrogen atom in a circularly polarized microwave field
}

\author{
Mercè Olléa ${ }^{\text {, Juan R. Pacha }}{ }^{\mathrm{a}, *}$ \\ ${ }^{a}$ Department of Mathematics. Universitat Politècnica de Catalunya, Barcelona, Spain
}

\begin{abstract}
We consider the Rydberg electron in a circularly polarized microwave field, whose dynamics is described by a Hamiltonian depending on one parameter, $K>0$. The corresponding Hamiltonian system of ODE has two equilibrium points $L_{1}$ (unstable for all $K$ and energy value $h\left(L_{1}\right)$ ) and $L_{2}$ (a center for $K<K_{\text {crit }}$ and a complex saddle for $K>K_{\text {crit }}$, with energy value $h\left(L_{2}\right)$ ). We study the Hamiltonian-Hopf bifurcation phenomena that take place for $K$ close to $K_{\text {crit }}$ around $L_{2}$. First, a local analysis based on the computation of the integrable normal form up to a finite order is carried out and the steps for the computation of this (resonant) normal form are explained in a constructive manner. The analysis of the normal form obtained allows: to claim the type of the Hopf bifurcation -supercritical-; to study the local behavior of the electron in a neighborhood of the equilibrium $L_{2}$ for the original non integrable Hamiltonian (as a perturbative approach from the integrable normal form); to obtain (approximations for) the parametrizations of the relevant invariant objects that take place due to the bifurcation (periodic orbits and invariant manifolds of $L_{2}$ ). We compute numerically such objects and analyse not only the local picture of the dynamics close to $L_{2}$, but also a global description of the dynamics and the effect of the Hopf bifurcation as well as other objects that organize the dynamics are discussed. We conclude that, for $K$ close to $K_{\text {crit }}$ and the energy level $h\left(L_{2}\right)$, the Hopf bifurcation has essentially no effect on the dynamics from a physical point of view. However, for bigger values of $K>K_{c r i t}$, the Hopf bifurcation has a dramatic effect: different kind of orbits coexist, mostly chaotic. Such orbits provide a ionization mechanism with several passages far from and close to $L_{2}$ before ionizing. Surprisingly enough, also robust confinement regions (where the electron remains confined for ever), exist in the middle of chaotic areas.
\end{abstract}

Keywords: Hamiltonian dynamical systems, Hopf bifurcation, periodic orbits and tori, chaotic regions, invariant manifolds

Accepted Manuscript. Final published version in Commun Nonlinear Sci Numer Simulat 62 (2018) 27-60.

(C) 2018. This manuscript version is made available under the CC-BY-NC-ND 4.0 license $\pi$ (cc) BY-NC-ND

\section{Introduction}

Within the classical mechanics context, we consider the hydrogen atom interaction with a circularly polarized microwave field, which we will refer to, from now on, as the CP problem. In suitable rotating variables, the CP problem is modeled by a Hamiltonian system with two degrees of freedom (assuming planar motion for the electron) that depends only on a parameter $K>0$.

The CP field is a quite well known problem in classical mechanics. Actually, the Hamiltonian of the CP problem may also be regarded as the Hamiltonian of an orbiting dust particle under radiation pressure, which is a restricted three-body-like problem, where a Coriolis term appears. This problem was already studied by Deprit [15] (he derived and analyzed a first order normal form for the Hamiltonian), and, later on, Cushman and Van der Meer [14] derived and studied a second order normal form. Not only is the CP problem relevant in this macroscale astronomy problem, but also in the microscale situation, where the expression of the Hamiltonian is formally the same, and which is the context of this paper. More specifically, there has been consistent interest in the interaction of a Rydberg atom with a circularly polarized (CP) microwave field in atomic physics. Actually Fu et al. [19] made the first experimental observations of the ionization of sodium Rydberg atoms by circularly (CP) and linearly (LP) polarized microwave fields and their experiments revealed a substantial higher threshold field for ionization in the case of CP rather than for a LP field.

In this paper we will focus on the particular case of the hydrogen atom submitted to a circularly polarized microwave field (shortly the CP problem). Many papers have been devoted to this problem and focused in particular, on the ionization mechanism for the electron. Concerning ionization, some controversy about the role of collisions, or paths close to collisions with the core, to explain a ionization mechanism for the electron has been present. We mention those authors that argue that collisions play a minor (irrelevant) role for ionization (see Zakrzewski and coworkers, [20] and [60]) and those that, on the contrary, argue that collisions are needed and play major (relevant) role for ionization (see Brunello et al. [10]). Other authors, by means of numerical and analytical techniques, also claim the existence of a new ionization process in which the electron picks up energy while it is far from the nucleus and then ionizes directly (see [6], [16], [37], [38], [44], [49]).

* corresponding author

Email addresses: merce.olle@upc.edu (Mercè Ollé), juan.ramon.pacha@upc.edu (Juan R. Pacha) 
On the analytical side, we also point out the perturbative approach dealing with integrable Hamiltonian systems which are good approximations for the description of the hydrogen atom in a circularly polarized microwave field -for low frequencies(see [22], [45] and [46]), or in crossed magnetic and electric fields -for low magnetic field- (see [30]).

So far we have mentioned papers published in the eighties and nineties. The interest for recollision (i.e., the electron can collide under intense laser force with the core) is at the heart of strong-field physics and continues in the twenties. Actually recollisions result from a subtle compromise between the action of the strong laser field (which leads the electron away in a swirling motion) and the Coulomb attraction (which tends to recall it). Moreover, the mechanisms for ionization as well as some dynamical aspects of the Hamiltonian system of ODE modelling the CP problem, remain still far from being well understood. In this sense, the application of techniques of the dynamical systems theory turn out to be quite useful, and the specific role of some invariant objects of the system of ODE is very revealing. First we highlight the paper by Kamor et al. [28] where the authors show how a family of key periodic orbits drives the recollision process in a strong circularly polarized laser field. Second, we mention the paper [5] where the authors consider weak amplitudes of the microwave field and study the relevant invariant objects in phase space that organize the dynamics, paying special attention to the role of the invariant stable and unstable manifolds of the hyperbolic Liapunov periodic orbit around the equilibrium point $L_{1}$. Such manifolds are the keystone to explain a mechanism for ionization. At this point, we mention a related paper -concerning the usefulness of invariant manifolds of periodic orbits-by Jaffé et al. [24], where they present the first application of transition state theory to the chaotic ionization of a hydrogen atom in crossed electric and magnetic fields. Finally, very recently, in [39], the author carries out a systematic numerical analysis of the orbits that eject from the core considering the CP problem. Many different types of orbits are obtained: regular/chaotic orbits that have several far excursions and close passages to the core, and finally either collide with it or ionize.

We finally remark that the references above are related to the hydrogen atom, so a single electron is considered. Of course, not only single electron ionization in the $\mathrm{CP}$ problem is of interest, but also double (and multiple) ionization -involving two or more electrons respectively-. We mention the papers [18] and [33] where experimental observations for the Magnesium and Helium cases to study double ionization are analysed.

From now on, we will focus our attention on the CP problem.

We first remark that the $\mathrm{CP}$ problem can be regarded as a perturbed Kepler problem (the CP problem becomes the Kepler problem in rotating coordinates for $K=0$ ) and, in fact, some features of the dynamics can be understood from the dynamics of the two body problem, when $K$ is small.

Concerning the Hamiltonian system of ODE for the CP problem, it is well known (see [5]) that it has two equilibrium points for any value of $K: L_{1}$ which is of type center $\times$ saddle, for all $K>0$, and $L_{2}$ which is a center $\times$ center for $K<K_{\text {crit }}$, being $K_{\text {crit }}=3^{-4 / 3} / 2 \approx 0.11556021$ a critical value, and a complex saddle for $K>K_{\text {crit }}$. The studies devoted to small values of $K$ and $K<K_{\text {crit }}$ reveal the existence of different kinds of solutions: confined, periodic, homoclinic, chaotic, escaping/ionizing among others. For the latter, it is known that there are fast escaping orbits and slowly erratic escaping orbits, in the sense that they make several excursions reaching small and big distances from the nucleus in an apparent random way such that it is difficult to predict when the electron will actually ionize. Barrabés et al. [5] were able to explain the mechanism for these kinds of orbits, based upon the invariant manifolds of some suitable periodic orbits. This was a main goal of that paper for $K<K_{\text {crit }}$ and the present paper is a natural continuation of it.

In this paper we study the dynamics of the CP problem also for the $K>K_{\text {crit }}$ case, and the fact that $L_{2}$ experiences the so called supercritical Hopf bifurcation when $K$ increases and crosses the critical value $K_{\text {crit }}$. In [17] the Hopf bifurcation for this problem was already mentioned and some preliminary numerical simulations concerning both cases $K<K_{\text {crit }}$ and $K>K_{\text {crit }}$ were carried out; see also [29] for an analytical approach. Our purpose is to describe the dynamics, not only from a local point of view, but from a global one and the consequences that this Hopf bifurcation has on the behavior of the solutions. This will require, for numerical simulations, that we will have to compute periodic orbits, their 2D associated invariant manifolds as well as the 2D invariant manifolds associated with the complex saddle equilibrium point $L_{2}$. Also suitable Poincaré sections will become very useful to describe the dynamics as well as to find confinement regions where the electron remains trapped for ever in the middle of apparent chaotic regions.

So our contribution in this paper is threefold:

(i) To apply an analytical procedure to transform the original and non-integrable Hamiltonian to a fourth order Hamiltonian normal form plus a remainder. The explicit steps are given and an algorithm that might be implemented for any order is explained (see remarks at the end of Appendix AppendixD, after the proof of Proposition 1). We point out that the papers dealing with the Hopf bifurcation typically consider an academic theoretical Hamiltonian, depending on generic parameters, with a suitable expression useful to carry out the normal form process, whereas here, we take the given Hamiltonian of the CP problem.

(ii) The analytical description of the dynamics of the integrable fourth order normal form for $K$ close to $K_{\text {crit }}$. Of course, this is a local approach due to the validity of the normal form in a small neighborhood of the equilibrium point $L_{2}$. In particular, the two families of periodic orbits (and their stability) around the stable $L_{2}$, for $K<K_{\text {crit }}$, as well as their merging to become one bifurcated detached family, when $L_{2}$ becomes a complex saddle for $K>K_{\text {crit }}$ is explained. Also the parametrizations of the manifolds of the unstable $L_{2}$ are derived. The CP problem is also considered in [29] to illustrate the Hamiltonian Hopf bifurcation. However, the treatment found there is perturbative, in the sense that the authors consider the Hamiltonian system in a neighborhood of $L_{2}$ and check that this equilibrium point changes its stability from center to complex saddle when the polarization parameter crosses a critical threshold; 
they unfold the normalized quadratic part of the Hamiltonian with two different terms that yields to the supercritical and subcritical bifurcation (see [8]), respectively. Here, we consider always the Hamiltonian of the CP problem, without any assumptions on the form of the higher order terms and carry out the computation of the normal form, in an explicit way, up to any given order.

Once the heavy mathematical analysis is carried out to obtain the normal form, the natural question that arises is: How do we use it? On the one hand, we can confirm that the type of bifurcation is supercritical (as the preliminary numerical simulations done in [17] seemed to indicate). On the other hand, from the analysis of the normal form itself we can predict how the dynamics will look like. Results are stated in Proposition 3. Of course, this prediction is true for $K$ very close to $K_{c r i t}$, but by means of numerical simulations, we are able to analyse the dynamics for bigger values of $K$.

(iii) So far the analytical part, next we focus on numerical simulations. On the one hand, we describe the Hopf bifurcation that takes places in the CP problem computing numerically all the invariant objects involved and showing the role they have in the dynamics. Although the analysis of the integrable normal form is valid only for a tiny neighborhood of $L_{2}$ and a small interval containing $K_{\text {crit }}$, the analytical computation of the normal form provides good (approximate) initial conditions of the main invariant objects appearing (periodic orbits and stable and unstable manifolds of $L_{2}$ ) in the Hopf bifurcation. These initial conditions are good seeds to be refined taking the original non-integrable Hamiltonian. For bigger values of $K$ and larger regions in the configuration plane (position coordinates for the electron), we take the original non-integrable Hamiltonian and make numerical simulations from it. More precisely, for the energy level of $L_{2}$, we describe how the 2D invariant manifolds of the equilibrium point $L_{2}$, for $K>K_{\text {crit }}$, provide a mechanism to explain a rich variety of orbits, including homoclinic orbits, multi-bump ones (with several close passages to the $L_{2}$ ), chaotic ones and even slow and fast ionization for the electron if $K$ is big enough. This is a first consequence of the Hopf bifurcation. Another effect is that the elliptic bifurcated periodic orbits (for $K$ close to $K_{c r i t}$ ) determine a region of confinement for ever in the middle of a chaotic area. It is worth mentioning that, for bigger values of $K$, the bifurcated obits become unstable, and, therefore such confinement vanishes. On the other hand, for values of the energy close to that of $L_{2}$ and varying $K>0$, we describe the dynamics, from a global point of view, for the original non integrable Hamiltonian. We explain how some periodic orbits, that exist regardless the Hopf bifurcation phenomena, the 2D invariant manifolds of the equilibrium point $L_{2}$, the 2D invariant manifolds of the Liapunov orbits around $L_{1}$, the bifurcated periodic orbits and 2D invariant tori organize the dynamics.

Remark 1. The CP problem is addressed here as classical Hamiltonian system. Quantum considerations are beyond the scope of this study. Nevertheless, it could be interesting to apply, somehow, quantization rules to the normal form. However, according to the observations in [29], though there exist methods to quantisize the Birkhoff-Gustavson normal form in the semi-classical approximation, the quantisation problem involving non semisimple resonances (as that taking in the Hopf bifurcation) is not yet completely understood. Even so, in the quoted paper, the authors manage to give, under some assumptions, the spectrum for the supercritical bifurcation. Readers interested in these aspects are referred to there.

The paper is organized as follows: first, in Section 2 we give the equations of motion of the Rydberg electron in the $\mathrm{CP}$ hydrogen atom, seen as a Hamiltonian system written in a rotating reference frame, as well as some general features of the model. Next, in Section 3 we describe the transformation from that initial Hamiltonian to a (resonant, integrable) normal form plus a (non integrable) remainder. Thus, after expanding the Hamiltonian locally around the equilibrium point $L_{2}$, normalize the quadratic terms by means of a symplectic linear change, and introduce complex coordinates, we revisit the nonlinear reduction process (the normal form computations). The results -for this case of the equilibrium $L_{2}$ with $K$ close to $K_{c r i t}-$ are set in Proposition 1, which tackles the solvability of the homological equations and gives the structure of the normal form. On its turn, in the same section, Proposition 2 states the real normal form that one obtains after changing back to real coordinates. In Section 4 we use this normal form to discuss the stability of the Rydberg electron at the equilibrium $L_{2}$ for values of the polarization parameter, $K$, near $K_{\text {crit }}$. In Section 5 the dynamics of the normal form is explored, as a way to get an (approximate and local) description of the phase space in a neighborhood of the equilibrium point. Section 6 is devoted to explain the numerical tools used in Section 7, where we present a detailed description of the dynamics from a global point of view, when $K<K_{\text {crit }}$ and when $K>K_{c r i t}$, for values of the energy $h$ close to the value at the equilibrium point $h\left(L_{2}\right)$, focusing our attention on the effects of the Hopf bifurcation. Conclusions are in Section 8.

In order to ease the reading of the paper, the more technical aspects have been deferred to the appendices. They require some more notation and extra definitions that can be found in Appendix AppendixA. Then, in Appendix AppendixB the details concerning the expansion of the Hamiltonian are given; the reduction to normalized complex coordinates is in Appendix AppendixC, and Appendix AppendixD is devoted to the proof of Proposition 1.

We remark that the numerical computations in Section 6 have been done with double precision and the integrations of the ODE carried out along the paper have used a Taylor method, through the software package provided by Jorba and Zou [27]. This is a robust method, both in speed and accuracy, and suitable for long time span integrations.

\section{The CP problem}

In this Section we present the equations of the problem and some basic properties related to equilibrium points, their stability, and some families of periodic orbits (PO) that play an important role in the dynamics of the problem. 
Let us consider the relative motion of the electron in a hydrogen atom subjected to a circularly polarized (CP) microwave, where the pulse of the microwave field is taken with a flat-top shape, i.e., the field amplitude is ramped up in time until it achieves a final, constant amplitude. Here we ignore the ramping and only consider the dynamics after the flat-top has been reached (see [10] for a discussion of the implications of the initial ramp).

The system of ordinary differential equations (ODE) of motion for the electron of the hydrogen atom (in the limit of an infinitely massive nucleus and in atomic units $m_{e}=\bar{h}=e=1$ ) subjected to a CP microwave field, and assumed to move in the plane $(X, Y)$ (position coordinates), is the following:

$$
\begin{aligned}
& \ddot{X}=-\frac{X}{R^{3}}-F \cos (\widetilde{\omega} s), \\
& \ddot{Y}=-\frac{Y}{R^{3}}-F \sin (\widetilde{\omega} s),
\end{aligned}
$$

where $R^{2}=X^{2}+Y^{2}, s$ is the time, ${ }^{\circ}=\frac{\mathrm{d}}{\mathrm{d} s}, \widetilde{\omega}$ is the angular frequency of the microwave field and $F>0$ is the field strength (see [10]). This system can be derived from the Hamiltonian

$$
\widetilde{H}(X, Y, \dot{X}, \dot{Y})=\frac{1}{2}\left(\dot{X}^{2}+\dot{Y}^{2}\right)-\frac{1}{R}+F(X \cos (\widetilde{\omega} s)+Y \sin (\widetilde{\omega} s)) .
$$

We take a rotating frame with the $\mathrm{CP}$ field, that is, $(x, y)$ coordinates such that

$$
\left(\begin{array}{l}
X \\
Y
\end{array}\right)=\left(\begin{array}{rr}
\cos (\widetilde{\omega} s) & -\sin (\widetilde{\omega} s) \\
\sin (\widetilde{\omega} s) & \cos (\widetilde{\omega} s)
\end{array}\right)\left(\begin{array}{l}
x \\
y
\end{array}\right)
$$

and the Eq. (1) become

$$
\begin{aligned}
& \ddot{x}-2 \widetilde{\omega} \dot{y}=\widetilde{\omega}^{2} x-\frac{x}{r^{3}}-F, \\
& \ddot{y}+2 \widetilde{\omega} \dot{x}=\widetilde{\omega}^{2} y-\frac{y}{r^{3}},
\end{aligned}
$$

where $r^{2}=x^{2}+y^{2}$. This system can be written in Hamiltonian form using

$$
H\left(x, y, p_{x}, p_{y}\right)=\frac{1}{2}\left(p_{x}^{2}+p_{y}^{2}\right)-\widetilde{\omega}\left(x p_{y}-y p_{x}\right)-\frac{1}{r}+F x
$$

where $\left(p_{x}, p_{y}\right)$ are the momenta defined by $p_{x}=\dot{x}-\widetilde{\omega} y$ and $p_{y}=\dot{y}+\widetilde{\omega} x$.

Now, we simplify this Hamiltonian re-scaling time and distances. We define a new time $t=\widetilde{\omega} s$ and consider the symplectic change of coordinates with multiplier $\widetilde{\omega}^{-1 / 3}$, more precisely,

$$
(x, y)=\widetilde{\omega}^{-2 / 3}(\bar{x}, \bar{y}), \quad\left(p_{x}, p_{y}\right)=\widetilde{\omega}^{1 / 3}\left(\bar{p}_{x}, \bar{p}_{y}\right) .
$$

The transformed Hamiltonian becomes in the new variables (for simplicity, we drop the bar and we keep the same names for the position and momentum coordinates),

$$
H=\frac{1}{2}\left(p_{x}^{2}+p_{y}^{2}\right)-x p_{y}+y p_{x}-\frac{1}{r}+K x
$$

where $K=F / \widetilde{\omega}^{4 / 3}>0$ is the unique parameter, $r^{2}=x^{2}+y^{2}$ and now $p_{x}=\dot{x}-y$ and $p_{y}=\dot{y}+x$, being ${ }^{\cdot}=\frac{\mathrm{d}}{\mathrm{d} t}$, $\mathrm{t}$ the new time. This is the Hamiltonian that will be considered from now on. We remark that it is autonomous and the constant value of the Hamiltonian $H=h$ over each solution is called the energy.

The associated Hamiltonian equations of the motion are

$$
\begin{aligned}
\dot{x} & =p_{x}+y, \\
\dot{y} & =p_{y}-x, \\
\dot{p}_{x} & =p_{y}-\frac{x}{r^{3}}-K, \\
\dot{p}_{y} & =-p_{x}-\frac{y}{r^{3}},
\end{aligned}
$$

which satisfy the symmetry

$$
\left(t, x, y, p_{x}, p_{y}\right) \longrightarrow\left(-t, x,-y,-p_{x}, p_{y}\right)
$$

This implies that, for each solution of the equations of motion, there also exists another one which is symmetric with respect to $y=0$ in configuration space $(x, y)$. In particular, periodic symmetric solutions with respect to $y=0$ intersect this axis perpendicularly twice. We use this property,in particular, to find families of periodic symmetric solutions. 
The CP problem in rotating coordinates has two equilibrium points, denoted by $L_{1}$ and $L_{2}$ and located in the $x$-axis at $x\left(L_{1}\right)<0$ and $x\left(L_{2}\right)>0$ respectively $\left(x\left(L_{i}\right), i=1,2\right.$ varying with $\left.K\right)$. Regarding their linear stability, the first one is of type saddle $\times$ center for all values of $K$, whereas the second one is of type center $\times$ center for

$$
K<K_{\text {crit }}=\frac{3^{-4 / 3}}{2} \approx 0.115560212391772
$$

and a complex saddle for $K>K_{\text {crit }}$.

It is worth noticing that, when the electron is not far away from the nucleus, the problem given by (5) may be regarded as a perturbed Kepler problem when the parameter $K$ is small, which is the case considered in [5]. Actually, from the rotating two-body problem, $K=0$, it is known that, for $h<-3 / 2$, there exists one family of retrograde periodic ones (denoted by $o_{r}$ ), family that persists when $K>0$ and we denote it in the same way.

Also for $K>0$ and small, since $L_{1}$ is of type saddle $\times$ center, and $L_{2}$ of type center $\times$ center (for $K<K_{\text {crit }}$ ), applying the Liapunov center theorem (see, for example [51], Chap. 2, or [34], Chap. 9), we obtain three families of periodic orbits (parameterized by the energy and known as Liapunov periodic orbits), one around $L_{1}\left(o l_{1}\right)$ and two around $L_{2}$ (one family existing for increasing values of the energy $h$, and the other family for decreasing values of $h$, both starting at $h\left(L_{2}\right)$ ).

One of the main goals of this paper is precisely to describe the Hopf bifurcation phenomenon that experiences the equilibrium point $L_{2}$ when $K$ increases and crosses the critical value $K_{\text {crit }}$.

For this purpose, a very useful theoretical approach will be to consider a lower (fourth) order normal form associated with the Hamiltonian. This is done in the next Section.

\section{Reduction to normal form around $L_{2}$}

This section is devoted to the reduction of the Hamiltonian (5), locally, around the equilibrium point $L_{2}$ to its normal form, for values of the polarization parameter $K$ close to the critical value $K_{\text {crit }}$ in (8).

As linearization shows, the stability type of this point changes from center to complex saddle as $K$ moves from $K<K_{\text {crit }}$ to $K>K_{\text {crit }}$ passing through a double (degenerate) center at $K=K_{\text {crit }}$. For this value of $K$ the characteristic exponents collide pairwise on the imaginary axis and the matrix of the linearized system has two pure imaginary eigenvalues, $\pm i \varpi$, $\varpi>0$; of algebraic multiplicity two, but geometric multiplicity one. This (parametric) resonance is known in the literature as 1:1 non semisimple resonance or, more briefly, as 1:-1 resonance and has drawn attention of many researchers (see [34], [50], [56], and references therein). The relevant features of the local dynamics can be gleaned from the resonant normal form around $L_{2}$ of the involved Hamiltonian (5).

Normal form reduction process embraces first, the expansion of the Hamiltonian at $L_{2}$. This is carried out in Subsection 3.2 (and in Appendix AppendixB); secondly, the linear reduction and complexification. This is discussed in Subsection 3.3 (and in Appendix AppendixC), where the quadratic part of the Hamiltonian is put in Williamson's normal form and then complex coordinates are introduced. Finally, the nonlinear reduction is described in Subsection 3.4. From a computational point of view, this process consists in setting and solving the so called homological equations to obtain both, the terms of the generating function of the transformation and the terms of the normal form. The solvability of the homological equations (for the current system with an equilibrium point near 1:-1 resonance) is stated in Proposition 1. Its proof, however, is a bit technical and has been moved to Appendix AppendixD. In the same Subsection 3.4, Proposition 2 gives the real normal form that will be analyzed in Section 4.

Before proceeding, we need to set the notation and give a few definitions. This is done below, in Subsection 3.1.

\subsection{Notation and definitions}

Given $R>0, \mathscr{D}_{R}$ will denote the polydisk,

$$
\mathscr{D}_{R}=\left\{(\nu, \alpha, \beta) \in \mathbb{C} \times \mathbb{C}^{2} \times \mathbb{C}^{2}:|\nu| \leq R^{2},|(\alpha, \beta)|_{\infty} \leq R\right\},
$$

where $|\cdot|,|\cdot|_{\infty}$ denote the modulus of a complex number and the supremum norm, respectively.

In expansions, it is often used multi-index notation for the monomials involved so, if $\nu \in \mathbb{C}, \alpha=\left(\alpha_{1}, \alpha_{2}\right), \beta=\left(\beta_{1}, \beta_{2}\right) \in$ $\mathbb{C}^{2} ; f_{\ell r_{1} r_{2} s_{1} s_{2}} \in \mathbb{C} ; \ell \in \mathbb{N}_{0}=\mathbb{N} \cup\{0\} ; r, s \in \mathbb{N}_{0}^{2}$, we write

$$
f_{\ell r s} \nu^{\ell} \alpha^{r} \beta^{s}=f_{\ell r_{1} r_{2} s_{1} s_{2}} \nu^{\ell} \alpha_{1}^{r_{1}} \alpha_{2}^{r_{2}} \beta_{1}^{s_{1}} \beta_{2}^{s_{2}}
$$

Hence, let $\mathbb{C}[[\nu, \alpha, \beta]]:=\mathbb{C}\left[\left[\nu, \alpha_{1}, \alpha_{2}, \beta_{1}, \beta_{2}\right]\right]$ be the ring of complex formal power series in $\nu \in \mathbb{C}, \alpha=\left(\alpha_{1}, \alpha_{2}\right) \in \mathbb{C}^{2}$, $\beta=\left(\beta_{1}, \beta_{2}\right) \in \mathbb{C}^{2}$. Therefore, $F \in \mathbb{C}[[\nu, \alpha, \beta]]$ if, and only if, it is a formal power series of type

$$
F=\sum f_{\ell r s} \nu^{\ell} \alpha^{r} \beta^{s}
$$

where the summation is performed over $\ell \in \mathbb{N}_{0}$ and $r, s \in \mathbb{N}_{0}^{2}$.

For any two $F, G \in \mathbb{C}[[\nu, \alpha, \beta]]$,

$$
\{F, G\}=\sum_{i=1,2}\left(\frac{\partial F}{\partial \alpha_{i}} \frac{\partial G}{\partial \beta_{i}}-\frac{\partial F}{\partial \beta_{i}} \frac{\partial G}{\partial \alpha_{i}}\right)
$$


is their standard Poisson bracket with respect to $(\alpha, \beta)$. Now, given $G \in \mathbb{C}[[\nu, \alpha, \beta]]$ fixed, we define $L_{G}:=\{\cdot, G\}$, so $L_{G} F=\{F, G\}$ for every $F \in \mathbb{C}[[\nu, \alpha, \beta]]$ and, recursively

$$
L_{G}^{0} F:=F, \quad L_{G}^{k} F:=L_{G}\left(L_{G}^{k-1} F\right),
$$

$k=1,2,3, \ldots$ For the monomials (9), we define their adapted or weighted degree by

$$
\operatorname{deg}\left\{\nu^{l} \alpha^{r} \beta^{s}\right\}:=2 \ell+|r|_{1}+|s|_{1},
$$

being $|\cdot|_{1}$ the usual 1-norm in $\mathbb{C}^{n}$. The approach to the reduction to normal form we present here involves the following spaces of homogeneous polynomials: $\mathbb{E}_{\sigma}[\nu, \alpha, \beta]$ will stand for the space of the homogeneous polynomials of (adapted) degree $\sigma=2,3, \ldots$ made up of monomials of type (9). Furthermore, for $\ell, M, N \in \mathbb{N}_{0}$ fixed, $\mathbb{E}_{\ell M N}[\ell, \alpha, \beta]$ will denote the subspace of $\mathbb{E}_{2 \ell+M+N}[\nu, \alpha, \beta]$ spanned also by monomials of type (9) with $M=r_{1}+r_{2}, N=s_{1}+s_{2}$. Clearly,

$$
\mathbb{E}_{\sigma}[\nu, \alpha, \beta]=\bigoplus_{2 \ell+M+N=\sigma} \mathbb{E}_{\ell M N}[\nu, \alpha, \beta] .
$$

Let $G(\nu, \alpha, \beta)$ be a real analytic function, and

$$
\dot{\alpha}_{j}=\frac{\partial G}{\partial \beta_{j}}(\nu, \alpha, \beta), \quad \dot{\beta}_{j}=-\frac{\partial G}{\partial \alpha_{j}}(\nu, \alpha, \beta),
$$

$j=1,2$; its associated Hamiltonian system (where $\nu \in \mathbb{C}$ is taken as a parameter), then

$$
\alpha_{j}(t)=\varphi_{j}^{G}\left(t ; \nu, \alpha^{0}, \beta^{0}\right), \quad \beta_{j}(t)=\psi_{j}^{G}\left(t ; \nu, \alpha^{0}, \beta^{0}\right)
$$

will denote its solution with initial conditions $\alpha_{j}(0)=\alpha_{j}^{0}, \beta_{j}(0)=\beta_{j}^{0} ; j=1,2$, and

$$
\Phi_{t}^{G}\left(\nu, \alpha^{0}, \beta^{0}\right)=(\alpha(t), \beta(t))=\left(\varphi^{G}\left(t ; \nu, \alpha^{0}, \beta^{0}\right), \psi^{G}\left(t ; \nu, \alpha^{0}, \beta^{0}\right)\right)
$$

its time-t flow. To simplify, for $t=1$, we shall write $\Phi^{G}$ instead of $\Phi_{1}^{G}$. Finally, given a function $f$ depending on a parameter $\nu \in \mathbb{C}: z \in \mathbb{C}^{m} \mapsto f(\nu, z) \in \mathbb{C}^{n}$, we define its $\nu$-extension, $f_{\nu}$, as

$$
(\nu, z) \in \mathbb{C} \times \mathbb{C}^{m} \rightarrow f_{\nu}(\nu, z)=(\nu, f(\nu, z)) \in \mathbb{C} \times \mathbb{C}^{n} .
$$

\subsection{Local coordinates at $L_{2}$}

The first step is to expand Hamiltonian (5) around the equilibrium point $L_{2}$, which is placed on the $x$ axis in the configuration space. Let $\delta$ denote the distance from the origin to the equilibrium point $L_{2}$. Therefore, from Eq. (6) we see on the one hand, that the position of $L_{2}$-in the phase space coordinates of the Hamiltonian (5)-is given by $\left(x, y, p_{x}, p_{y}\right)=(\delta, 0,0, \delta)$ and, on the other hand, that $\delta$ is the only positive solution of the equation

$$
x^{3}-K x^{2}-\operatorname{sign}(x)=0,
$$

for $x>0$. Notice that $\delta$ depends on $K$ and let $\delta_{0}$ denote the value of $\delta$ corresponding to the critical value of the parameter $K, K_{\text {crit }}$. An straightforward computation yields,

$$
\delta_{0}=\frac{3^{2 / 3}}{2} .
$$

Moreover, we shall define the parameter $\mu$ as the difference:

$$
\mu:=\frac{1}{\delta_{0}}-\frac{1}{\delta}
$$

From (14) - (16) one can correlate $K$ as a function of $\mu$ (see also equations (B.7)). Then it can be seen that $K_{\text {crit }}$ corresponds to $\mu=0$ and that $K$ crosses the value $K_{\text {crit }}$ from $K<K_{\text {crit }}$ to $K>K_{\text {crit }}$ as $\mu$ increases from $\mu<0$ to $0<\mu<1 / \delta_{0}$. Therefore, the equilibrium point $L_{2}$ changes from a center to a complex saddle as $\mu$ crosses the zero from negative to positive values. The symplectic change,

$$
x=\delta\left(1+x_{1}\right), \quad y=\delta x_{2}, \quad p_{x}=\frac{y_{1}}{\delta}, \quad p_{y}=\delta+\frac{y_{2}}{\delta},
$$

moves the equilibrium point to the origin. In vector notation, we shall write $x=\left(x_{1}, x_{2}\right), y=\left(y_{1}, y_{2}\right)$ for the new position and momentum, so $(x, y)=\left(x_{1}, x_{2}, y_{1}, y_{2}\right)$ stands for the new coordinates. Then, applying the shift (17) we define the new Hamiltonian $\mathcal{H}(\mu, x, y)$ as,

$$
\mathcal{H}(\mu, x, y):=H\left(\delta\left(1+x_{1}\right), \delta x_{2}, \frac{y_{1}}{\delta}, \delta+\frac{y_{2}}{\delta}\right)-H\left(\delta_{0}, 0,0, \delta_{0}\right) .
$$


Note that, in addition, we subtract $H\left(\delta_{0}, 0,0, \delta_{0}\right)$, which is the energy of the equilibrium point $L_{2}$ at resonance (i.e., for $\mu=0$ ). Of course this will not affect the dynamics of the associated Hamiltonian system. The next step is to expand Hamiltonian (18) as a sum of the form,

$$
\mathcal{H}(\mu, x, y)=\mathcal{H}_{2}(\mu, x, y)+\mathcal{H}_{3}(\mu, x, y)+\cdots+\mathcal{H}_{\sigma}(\mu, x, y)+\ldots
$$

with $\mathcal{H}_{\sigma} \in \mathbb{E}_{\sigma}[\mu, x, y]$ for $\sigma=2,3, \ldots$ In Appendix AppendixB, it is shown that, starting from $\mathcal{H}_{2}$,

$$
\mathcal{H}_{2}(\mu, x, y)=\left(2+\delta_{0}^{3}\right) \mu+\frac{1}{2 \delta_{0}^{2}}\left(y_{1}^{2}+y_{2}^{2}\right)+x_{2} y_{1}-x_{1} y_{2}-\frac{1}{\delta_{0}}\left(x_{1}^{2}-\frac{x_{2}^{2}}{2}\right),
$$

the computation of the higher degree terms, $\mathcal{H}_{\sigma}(\mu, x, y), \sigma=3,4, \ldots$, can be carried out, recursively, using the properties of Legendre polynomials (see equations (B.8) and (B.9) therein).

Remark 2. We point out that the parameter $\mu$ in Hamiltonian (18) can be thought of -rather than a parameter-as the action of a 3 -degree of freedom Hamiltonian that does not depend explicitly on the conjugate angle, $\theta$ (which is then a cyclic coordinate). Hence, one can adapt the reduction in [41], that led to a suspension of the Sokolskil normal form around a 1:-1 resonant periodic orbit (see [53], and also Chap. 10 in [34] and references therein). That justifies the double weight of the "action" coordinate -the parameter $\mu$ in the problem at hand- in the definition (10) of adapted degree.

\subsection{Linear reduction and complexification of the Hamiltonian system}

The second step is to reduce the quadratic part of the Hamiltonian. This issue goes back to the three seminal papers of J. Williamson ([57], [58], [59]). A constructive approach to the subject, giving a method for finding canonical forms for both, Hamiltonian (infinitesimally symplectic) and symplectic matrices, can be found in [31] (see references therein for an account of other relevant contributions). Lemma 1 in Appendix AppendixC gives a linear canonical change

$$
z=\mathcal{U}(\zeta):=S \zeta
$$

$S \in \operatorname{Sp}(4, \mathbb{R}) ; \zeta=(\xi, \eta), z=(x, y)$, with $\xi=\left(\xi_{1}, \xi_{2}\right), \eta=\left(\eta_{1}, \eta_{2}\right), x=\left(x_{1}, x_{2}\right), y=\left(y_{1}, y_{2}\right) \in \mathbb{R}^{2} ;$ that reduces the quadratic part $\mathcal{H}_{2}(\mu, x, y)$ of Hamiltonian (19) to its Williamson's normal form. Moreover, to ease the resolution of the homological equations, we consider complex coordinates of the type

$$
\xi_{1}=\frac{q_{1}-p_{2}}{\sqrt{2}}, \quad \xi_{2}=-\frac{q_{1}+p_{2}}{\mathrm{i} \sqrt{2}}, \quad \eta_{1}=\frac{q_{2}+p_{1}}{\sqrt{2}}, \quad \eta_{2}=-\frac{q_{2}-p_{1}}{\mathrm{i} \sqrt{2}} .
$$

One can check that (20) is a symplectic change. Let us denote it by $\zeta=\mathcal{V}(w)$; now with $w=(q, p) ; q=\left(q_{1}, q_{2}\right)$, $p=\left(p_{1}, p_{2}\right) \in \mathbb{C}^{2}$. Therefore, if we define the composition of both changes as $\Xi:=\mathcal{U} \circ \mathcal{V}$ and substitute $z$ by $\Xi(w)$ in $(19)$, the expansion in $(\mu, q, p)$ of the transformed Hamiltonian, $\mathscr{H}$, casts

$$
\mathscr{H}(\mu, q, p):=\mathcal{H}(\mu, \Xi(w))=\mathscr{H}_{2}(\mu, q, p)+\mathscr{H}_{3}(\mu, q, p)+\cdots+\mathscr{H}_{\sigma}(\mu, q, p)+\ldots,
$$

with

$$
\mathscr{H}_{2}(\mu, q, p):=\tilde{\alpha} \mu+\mathrm{i} \varpi\left(q_{1} p_{1}+q_{2} p_{2}\right)+q_{2} p_{1} .
$$

(see Section 4 for the current value of the coefficients $\tilde{\alpha}$ and $\varpi$ ).

\subsection{Nonlinear reduction. Homological equations}

The third step in the computation of the the normal form of the Hamiltonian (5) at the equilibrium $L_{2}$ consists in reducing the terms of degree higher than 2 in the expansion of $\mathscr{H}$; or, alternatively, if we consider $(21)$ as though it were the expansion of our starting Hamiltonian, to compute its normal form at $(q, p)=(0,0)$ with respect to $\mathscr{H}_{2}$. Here we sketch how effective computations can be performed up to any arbitrary degree $s \geq 3$. Actually, the outcome depends on which terms can and cannot be removed form the Hamiltonian -and so, remain in the normal form -or, in other words, on the solvability of the homological Eq. (25), for $\sigma \geq 3$. This point is stated in Proposition 1. Moreover, the real normal form, used later on to investigate the local dynamics, comes up in Proposition 2.

So, starting from Hamiltonian (21) and naming $\left(q^{(0)}, p^{(0)}\right)=(q, p)$ to the original (complex) coordinates, the normalization is carried out degreewise by successive composition of the symplectic changes $\left(q^{(\sigma-3)}, p^{(\sigma-3)}\right)=\Phi^{G_{\sigma}}\left(\mu, q^{(\sigma-2)}, p^{(\sigma-2)}\right)$, for $\sigma=3,4, \ldots$, where $\Phi^{G_{\sigma}}$ is the time- 1 flow of $G_{\sigma}$ (see notation in Subsection 3.1 above).

Thus, the first step consists in finding a pair $G_{3}, Z_{3} \in \mathbb{E}_{3}\left[\mu, q^{(1)}, p^{(1)}\right]$ that fulfills the equation,

$$
L_{Z_{2}} G_{3}+Z_{3}=\mathscr{H}_{3}^{(0)} \text {, }
$$

with $Z_{2}=\mathscr{H}_{2}, \mathscr{H}_{3}^{(0)}=\mathscr{H}_{3}$; then, the change generated by $G_{3},\left(q^{(0)}, p^{(0)}\right)=\Phi^{G_{3}}\left(\mu, q^{(1)}, p^{(1)}\right)$, transforms the Hamiltonian (21) to its $3^{\text {rd }}$ order (degree) reduced form, that reads

$$
\begin{gathered}
\mathscr{H}^{(1)}\left(\mu, q^{(1)}, p^{(1)}\right)=\mathscr{H}^{(0)}\left(\mu, \Phi^{G_{3}}\left(\mu, q^{(1)}, p^{(1)}\right)\right)= \\
Z_{2}\left(\mu, q^{(1)}, p^{(1)}\right)+Z_{3}\left(\mu, q^{(1)}, p^{(1)}\right)+\mathscr{H}_{4}^{(1)}\left(\mu, q^{(1)}, p^{(1)}\right)+\ldots \\
\cdots+\mathscr{H}_{\sigma}^{(1)}\left(\mu, q^{(1)}, p^{(1)}\right)+\ldots
\end{gathered}
$$


From here, proceeding inductively for $\sigma=4,5, \ldots$, up to $\sigma=s$,

$$
\begin{array}{r}
\mathscr{H}^{(\sigma-2)}\left(\mu, q^{(\sigma-2)}, p^{(\sigma-2)}\right)=\mathscr{H}^{(\sigma-3)}\left(\mu, \Phi^{G_{\sigma}}\left(\mu, q^{(\sigma-2)}, p^{(\sigma-2)}\right)\right)=Z_{2}\left(\mu, q^{(\sigma-2)}, p^{(\sigma-2)}\right)+Z_{3}\left(\mu, q^{(\sigma-2)}, p^{(\sigma-2)}\right)+\ldots \\
\cdots+Z_{\sigma}\left(\mu, q^{(\sigma-2)}, p^{(\sigma-2)}\right)+\mathscr{H}_{\sigma+1}^{(\sigma-2)}\left(\mu, q^{(\sigma-2)}, p^{(\sigma-2)}\right)+\mathscr{H}_{\sigma+2}^{(\sigma-2)}\left(\mu, q^{(\sigma-2)}, p^{(\sigma-2)}\right)+\ldots \quad(24)
\end{array}
$$

provided $G_{\sigma}, Z_{\sigma} \in \mathbb{E}_{\sigma}\left[\mu, q^{(\sigma-2)}, p^{(\sigma-2)}\right]$ satisfy

$$
L_{Z_{2}} G_{\sigma}+Z_{\sigma}=\mathscr{H}_{\sigma}^{(\sigma-3)} .
$$

Eqs. (23) and (25) are the homological equations corresponding to the order (degree) 3 and $\sigma=4, \ldots, s$, respectively; hence, at each fixed (adapted) degree $\sigma=3,4, \ldots, s$, to solve (25) one tries to find a generating function, $G_{\sigma}$, such that $L_{Z_{2}} G_{\sigma}=\mathscr{H}_{\sigma}^{(\sigma-3)}$. If this can be done, then on can set $Z_{\sigma}=0$; otherwise it must be added a complementary (or resonant) term $Z_{\sigma}$ to make the equation be valid.

Now, assume that at the $(\sigma-2)^{\text {th }}$ step, $G_{\sigma}$ and $Z_{\sigma}$ have been determined from (25). To go on with the next iteration, one needs to transform the current Hamiltonian, $\mathscr{H}^{(\sigma-3)}$, by the time-1 flow of the Hamiltonian system associated to $G_{\sigma}$. This transformation is carried out through the successive application of Poisson brackets,

$$
\mathscr{H}^{(\sigma-2)}=\mathscr{H}^{(\sigma-3)}+\frac{1}{1 !} L_{G_{\sigma}} \mathscr{H}^{(\sigma-3)}+\cdots+\frac{1}{k !} L_{G_{\sigma}}^{k} \mathscr{H}^{(\sigma-3)}+\ldots
$$

We note that, as the successive changes applied are near-identity transformations (see Remark AppendixD.8 in Appendix AppendixD), the first $\sigma-2$ terms, $Z_{2}, Z_{3}, \ldots, Z_{\sigma-1}$ of $\mathscr{H}^{(\sigma-3)}$, remain the same in $\mathscr{H}^{(\sigma-2)}$ (as can be noted in (24)).

Thus, proceeding in this way up to order $s \geq 4$, the final Hamiltonian, $\mathscr{H}^{(s-2)}$, is the sum $\mathscr{H}^{(s-2)}=Z^{(s)}+\mathscr{H}_{>s}$, where

$$
Z^{(s)}=Z_{2}+Z_{3}+Z_{4}+\cdots+Z_{s}
$$

is the normal form (up to degree $s$ ), made up of resonant terms; whilst

$$
\mathscr{H}_{>s}=\mathscr{H}_{s+1}^{(s-2)}+\mathscr{H}_{s+2}^{(s-2)}+\ldots
$$

is the remainder, that holds higher order terms.

Remark 3. In Proposition 1 we shall denote the subspaces

$$
\mathbb{E}_{\sigma}\left[\mu, q^{(\sigma-2)}, p^{(\sigma-2)}\right], \mathbb{E}_{\sigma}^{\mathcal{S}}\left[\mu, q^{(\sigma-2)}, p^{(\sigma-2)}\right], \quad \mathbb{E}_{\ell M N}\left[\mu, q^{(\sigma-2)}, p^{(\sigma-2)}\right], \quad \text { and } \mathbb{E}_{\ell M N}^{\mathcal{S}}\left[\mu, q^{(\sigma-2)}, p^{(\sigma-2)}\right]
$$

(see Section 3.1 and Appendix AppendixA for their definitions) just by $\mathbb{E}_{\sigma}, \mathbb{E}_{\sigma}^{\mathcal{S}}, \mathbb{E}_{\ell M N}$ and $\mathbb{E}_{\ell M N}^{\mathcal{S}}$ respectively, without specifying the coordinates $\mu, q^{(\sigma-2)}, p^{(\sigma-2)}$. On the other hand, we shall also stop using the super-indices $(\sigma-2)$ (or the corresponding ones) on the positions $q=\left(q_{1}, q_{2}\right)$ and momenta $p=\left(p_{1}, p_{2}\right)$. In both cases, it should be clear which coordinates are being used at each step.

Proposition 1. Let us consider $\mathscr{H}_{2}$ in (22), the decomposition of $\mathbb{E}_{\sigma}$ into $\mathbb{E}_{\sigma}=\mathbb{E}_{\sigma}^{0} \oplus \mathbb{E}_{\sigma}^{+}$, with

$$
\mathbb{E}_{\sigma}^{0}=\bigoplus_{2 \ell+2 M=\sigma} \mathbb{E}_{\ell M M}, \quad \mathbb{E}_{\sigma}^{+}=\bigoplus_{\substack{2 \ell+M+N=\sigma \\(M \neq N)}} \mathbb{E}_{\ell M N},
$$

so that $\mathbb{E}_{\sigma}^{0}=\{0\}$ if $\sigma$ is odd. Given $\mathscr{H}_{\sigma}^{(\sigma-3)}, \sigma \geq 3$, we split it as

$$
\mathscr{H}_{\sigma}^{(\sigma-3)}=\dot{\mathscr{H}}_{\sigma}^{(\sigma-3)}+\hat{\mathscr{H}}_{\sigma}^{(\sigma-3)}, \quad \dot{\mathscr{H}}_{\sigma}^{(\sigma-3)} \in \mathbb{E}_{\sigma}^{0}, \quad \hat{\mathscr{H}}_{\sigma}^{(\sigma-3)} \in \mathbb{E}_{\sigma}^{+} .
$$

Then, we have:

(a) There exists a unique $G_{\sigma}^{+} \in \mathbb{E}_{\sigma}^{+}$; such that $L_{Z_{2}} G_{\sigma}^{+}=\hat{\mathscr{H}}_{\sigma}^{(\sigma-3)}$. Moreover, $G_{\sigma}^{+} \in \mathbb{E}_{\sigma}^{\mathcal{S}}$.

(b) If $\sigma$ is even, there is a real coefficients homogeneous polynomial $\mathcal{Z}_{\sigma}(u, v, w)$ of standard degree $\sigma / 2$, which is uniquely defined in terms of $\dot{\mathscr{H}}_{\sigma}^{(\sigma-3)}$, such that if we set

$$
Z_{\sigma}(\mu, q, p):=\mathcal{Z}_{\sigma}\left(\mu, q_{1} p_{2}, \mathrm{i}\left(q_{1} p_{1}+q_{2} p_{2}\right) / 2\right)
$$

then $Z_{\sigma} \in \mathbb{E}_{\sigma}^{0} \cap \mathbb{E}_{\sigma}^{\mathcal{S}}$ and there is $G_{\sigma}^{0} \in \mathbb{E}_{\sigma}^{0}$ verifying $L_{Z_{2}} G_{\sigma}^{0}+Z_{\sigma}=\mathscr{\mathscr { H }}_{\sigma}^{(\sigma-3)}$. Moreover, $G_{\sigma}^{0}$ can be chosen so that $G_{\sigma}^{0} \in \mathbb{E}_{\sigma}^{\mathcal{S}}$.

Therefore, if the degree $\sigma$ is odd $G_{\sigma}:=G_{\sigma}^{+}$and $Z_{\sigma}:=0$ solve Eq. (25) in $\mathbb{E}_{\sigma}^{\mathcal{S}}$; otherwise, if $\sigma$ is even so does $G_{\sigma}:=G_{\sigma}^{+}+G_{\sigma}^{0}$ and $Z_{\sigma}$ given by (27).

PROOF. For a proof of Proposition 1 see Appendix AppendixD. 
The normal form transformation. Now, let $\mathcal{V}$ be the complexification $(20)$ and let $\chi(\mu, \widetilde{z})$ be given by $\chi(\mu, \widetilde{z}):=\left(\mu, \mathcal{V}^{-1}(\widetilde{z})\right)$; $\widetilde{z}=(\widetilde{x}, \widetilde{y})$, with $\widetilde{x}=\left(\widetilde{x}_{1}, \widetilde{x}_{2}\right), \widetilde{y}=\left(\widetilde{y}_{1}, \widetilde{y}_{2}\right) \in \mathbb{C}^{2}$. Proceeding as in [41] (see Proposition 7.1 there), it can be shown that, for any integer $s \geq 3$ fixed, and any $\kappa_{*}>1$ given, there exists $R_{s}>0$ such that the $\mu$-extension (see (13)), $\widetilde{\Phi}_{\mu}$, of

$$
\widetilde{\Phi}:=\mathcal{U} \circ \Phi^{G_{3} \circ \chi} \circ \Phi_{\mu}^{G_{4} \circ \chi} \circ \cdots \circ \Phi_{\mu}^{G_{s} \circ \chi}
$$

(here $\Phi_{\mu}^{G_{\sigma} \circ \chi}$ are the $\mu$-extensions of $\Phi^{G_{\sigma} \circ \chi}$ for $\left.\sigma=4, \ldots, s\right)$, is a real analytic function in the polydisk $\mathscr{D}_{R}$, with $\widetilde{\Phi}_{\mu}\left(\mathscr{D}_{R}\right) \subseteq$ $\mathscr{D}_{\kappa_{*} R}, 0<R<R_{s}$. Thus, Proposition 2 below follows then these last considerations and from Proposition 1.

Proposition 2 (Sokolski1̌'s normal form). Consider the $2 D$ of freedom real analytic Hamiltonian $\mathcal{H}$ in (19), depending on a parameter $\mu \in \mathbb{R}$, which has no linear terms in $(x, y)$, so the origin $O:(x, y)=(0,0)$ is an equilibrium point for every $\mu$. Assume that, for some $\mu_{*}<0, \mu^{*}>0$, its quadratic part, $\mathcal{H}_{2}$, is a center for $\mu_{*}<\mu<0$, has a non semi-simple 1:-1 resonance for $\mu=0$ (i.e., its characteristic exponents are pure imaginary with algebraic multiplicity two and geometric multiplicity one), and a complex-saddle for $0<\mu<\mu^{*}$. Then, given $s \in \mathbb{N}, s \geq 4$ even, there exists $\mu_{0}$, $0<\mu_{0} \leq$ $\min \left\{\left|\mu_{*}\right|, \mu^{*}\right\}$, such that: (i) for every $\mu,|\mu|<\mu_{0}$, the symplectic transformation (28), $(\tilde{x}, \tilde{y}) \mapsto(x, y)=\tilde{\Phi}(\mu, \tilde{x}, \tilde{y})$, is a real analytic diffeomorphism defined in a neighborhood of $\widetilde{O}:(\tilde{x}, \tilde{y})=(0,0)$, and (ii) the transformed Hamiltonian

$$
\begin{aligned}
& \widetilde{\mathcal{H}}^{(s-2)}(\mu, \tilde{x}, \tilde{y})=\mathcal{H}(\mu, \widetilde{\Phi}(\mu, \tilde{x}, \tilde{y})) \\
& \quad=\tilde{\alpha} \mu+\frac{\epsilon}{2}\left(\tilde{y}_{1}^{2}+\tilde{y}_{2}^{2}\right)+\varpi\left(\tilde{y}_{1} \tilde{x}_{2}-\tilde{y}_{2} \tilde{x}_{1}\right)+\sum_{\kappa=2}^{s / 2} \sum_{\ell+i+j=\kappa} \widetilde{Z}_{\ell i j}^{(2 \kappa)} \mu^{\ell}\left(\tilde{y}_{1} \tilde{x}_{2}-\tilde{y}_{2} \tilde{x}_{1}\right)^{i}\left(\tilde{x}_{1}^{2}+\tilde{x}_{2}^{2}\right)^{j}+\widetilde{\mathcal{H}}_{>s}(\mu, \tilde{x}, \tilde{y}),
\end{aligned}
$$

is also real analytic in that neighborhood. The Hamiltonian (29) is the sum, $\widetilde{\mathcal{H}}^{(s-2)}=\widetilde{Z}_{2}+\widetilde{Z}_{4}+\cdots+\widetilde{Z}_{s}+\widetilde{\mathcal{H}}_{>s}$, of

(1) an integrable part, the normal form, $\widetilde{Z}^{(s)}=\widetilde{Z}_{2}+\widetilde{Z}_{4}+\widetilde{Z}_{6}+\cdots+\widetilde{Z}_{s}$, with a quadratic (with respect to the adapted degree) term given by

$$
\widetilde{Z}_{2}(\mu, x, y)=\tilde{\alpha} \mu+\frac{\epsilon}{2}\left(\tilde{y}_{1}^{2}+\tilde{y}_{2}^{2}\right)+\varpi\left(\tilde{y}_{1} \tilde{x}_{2}-\tilde{y}_{2} \tilde{x}_{1}\right)
$$

being $\epsilon=\operatorname{sign}(\Delta)$ (with $\Delta$ defined in Lemma 1), and higher order terms $\widetilde{Z}_{4}, \widetilde{Z}_{6}, \ldots, \widetilde{Z}_{s}$, that are polynomials in $\mu$, $\tilde{y}_{1} \tilde{x}_{2}-\tilde{y}_{2} \tilde{x}_{1}, \tilde{x}_{1}^{2}+\tilde{x}_{2}^{2}$ of (ordinary) degree $k=2,3, \ldots, s / 2$-so homogeneous polynomials of even (adapted) degree $\sigma=4,6, \ldots, s$ in $\mu, \tilde{x}, \tilde{y}-$. We write,

$$
\widetilde{Z_{\sigma}}(\mu, \tilde{x}, \tilde{y})=\sum_{\ell+i+j=\sigma / 2} \widetilde{Z}_{\ell i j}^{(\sigma)} \mu^{\ell}\left(\tilde{y}_{1} \tilde{x}_{2}-\tilde{y}_{2} \tilde{x}_{1}\right)^{i}\left(\tilde{x}_{1}^{2}+\tilde{x}_{2}^{2}\right)^{j},
$$

(2) and the non normalized part, the remainder, $\widetilde{\mathcal{H}}_{>s}$, that begins with terms of degree $>s$.

Remark 4. We note that the normal form in the above proposition is slightly different from the one that appears in [53], [54], but coincides with that in [34], [50] and [56]. More remarks concerning the normal form are given at the end of Appendix AppendixD.

\section{Stability of the Rydberg's electron at the Hamiltonian Hopf bifurcation}

Here we discuss the stability of the equilibrium $O:(x, y)=(0,0)$ of the Hamiltonian (19) and hence, of the equilibrium $L_{2}$ : $\left(x, y, p_{x}, p_{y}\right)=(\delta, 0,0, \delta)$-with $\delta=\delta(\mu)$ given in (B.7)-, of the Hamiltonian (5) corresponding to the CP problem. Next we claim, in Theorem 1, that at the equilibrium $\widetilde{O}$ of Hamiltonian (29) (correspondingly, at $L_{2}$ of Hamiltonian (5)), it takes place a supercritical Hamiltonian Hopf bifurcation as $\mu(K)$ moves from $\mu<0\left(K<K_{\text {crit }}\right)$ to $\mu>0$ ( $\left.K>K_{\text {crit }}\right)$, crossing the critical value $\mu=0\left(K=K_{\text {crit }}\right)$. Finally, we describe the quasiperiodic bifurcation phenomena that take place around both, the Liapunov elliptic periodic orbits (for $\mu<0$ ) and the bifurcated elliptic periodic orbits (for $\mu \geq 0$ ). These items are summarized in Proposition 3.

The computations carried out in Examples 3 and 4 in Appendix AppendixD yield, after changing back to real coordinates using the inverse of the complexification (20), that is,

$$
q_{1}=\frac{\tilde{x}_{1}-\mathrm{i} \tilde{x}_{2}}{\sqrt{2}}, \quad q_{2}=\frac{\tilde{y}_{1}-\mathrm{i} \tilde{y}_{2}}{\sqrt{2}}, \quad p_{1}=\frac{\tilde{y}_{1}+\mathrm{i} \tilde{y}_{2}}{\sqrt{2}}, \quad p_{2}=-\frac{\tilde{x}_{1}+\mathrm{i} \tilde{x}_{2}}{\sqrt{2}},
$$

to the reduced real analytic Hamiltonian $\widetilde{\mathcal{H}}^{(2)}(\mu, \tilde{x}, \tilde{y})=\widetilde{Z}^{(4)}(\mu, \tilde{x}, \tilde{y})+\widetilde{\mathcal{H}}_{>4}(\mu, \tilde{x}, \tilde{y})$, where the fourth-degree normal form, $\widetilde{Z}^{(4)}$, looks as

$$
\begin{aligned}
\widetilde{Z}^{(4)}(\mu, \tilde{x}, \tilde{y})= & \tilde{\alpha} \mu+\tilde{\beta} \mu^{2}+\frac{1}{2}\left(\tilde{y}_{1}^{2}+\tilde{y}_{2}^{2}\right)+\omega(\mu)\left(\tilde{y}_{1} \tilde{x}_{2}-\tilde{y}_{2} \tilde{x}_{1}\right)-\frac{\varepsilon(\mu)}{2}\left(\tilde{x}_{1}^{2}+\tilde{x}_{2}^{2}\right) \\
& +A\left(\tilde{x}_{1}^{2}+\tilde{x}_{2}^{2}\right)^{2}+B\left(\tilde{x}_{1}^{2}+\tilde{x}_{2}^{2}\right)\left(\tilde{y}_{1} \tilde{x}_{2}-\tilde{y}_{2} \tilde{x}_{1}\right)+C\left(\tilde{y}_{1} \tilde{x}_{2}-\tilde{y}_{2} \tilde{x}_{1}\right)^{2}
\end{aligned}
$$


with coefficients,

$$
\begin{aligned}
& \tilde{\alpha}=\frac{25}{8}, \\
& \tilde{\beta}=\frac{27}{32} 3^{2 / 3}, \\
& \varpi=\frac{\sqrt{5}}{3}, \\
& \omega(\mu)=\varpi+\tilde{\gamma} \mu, \\
& A=\frac{7}{1250} 3^{2 / 3} \text {, } \\
& B=-\frac{6}{625} 3^{2 / 3} \sqrt{5} \text {, } \\
& \tilde{\gamma}=\frac{14}{25} 3^{2 / 3} \sqrt{5}, \\
& \varepsilon(\mu)=\frac{6}{5} 3^{2 / 3} \mu, \\
& C=-\frac{88}{28125} 3^{2 / 3} \text {. }
\end{aligned}
$$

and the corresponding Hamiltonian system is

$$
\dot{\tilde{x}}=\partial_{\tilde{y}} \widetilde{Z}^{(4)}(\mu, \tilde{x}, \tilde{y}), \quad \dot{\tilde{y}}=-\partial_{\tilde{x}} \widetilde{Z}^{(4)}(\mu, \tilde{x}, \tilde{y}) .
$$

Regarding the linear stability of the origin, it is checked at once that the characteristic exponents (the eigenvalues of the Jacobian matrix of system (33)) are

$$
\alpha_{ \pm}= \pm(\mathrm{i} \varpi+\sqrt{\varepsilon(\mu)})+\mathcal{O}(\mu), \quad \quad \beta_{ \pm}= \pm(\mathrm{i} \varpi-\sqrt{\varepsilon(\mu)})+\mathcal{O}(\mu) .
$$

Hence, as it was already mentioned in Section 3.2, for $\mu<0$ the equilibrium $\widetilde{O}:(\tilde{x}, \tilde{y})=(0,0)$ of the Hamiltonian (29) is a center, so its characteristic exponents are pure imaginary; for $\mu=0$ the characteristic exponents collide on the imaginary axis and leave off, pairwise, the imaginary axis to the complex plane for $\mu>0$. Therefore, the stability of the point $\widetilde{O}$ changes from a center to a complex saddle through a degenerate (in fact, a non semisimple) center.

(Nonlinear) Stability of the equilibrium. The stability of the equilibrium $\widetilde{O}$ is determined by the signs of the parameter $\mu$, the coefficient $A$ and the fourth-degree normal form (31) -we recall, see (32), that in the current case, $A=7 \times 3^{2 / 3} / 1250>0$ . The discussion of all possible cases can be found, for example, in [32]. Thus, as pointed out there, for $A>0$ and $\mu<0$ the characteristic exponents of $\widetilde{O}$ are pure imaginary, no low order resonances are present, and the conditions of of Arnold theorem (see [2], Chap. 5) are met, so the equilibrium $\widetilde{O}$ is stable. For $A>0$ and $\mu>0, \widetilde{O}$ is a complex saddle and, clearly, it is unstable. For $\mu=0, \widetilde{O}$ is still elliptic but a 1:-1 non semisimple resonance shows up, and its stability cannot be determined so straightforward. This issue has had a historically relevant motivation in the field of Celestial Mechanics: the concern about the Liapunov stability of Lagrange triangular points $L_{4}, L_{5}$ of the Restricted Circular Three Body Problem for the Routh critical mass ratio.

The first results in this direction were achieved in [53], proving formal stability of the equilibrium $\widetilde{O}$ for $A>0$ (and its instability for $A<0$ ). Later, the same author tackled the Liapunov stability for $A>0$ in [54] (see the comments in [32] plus the observations and references at the end of Sec. 6 of Chap. 13 in [34]). More recent proofs are found in [3] -where the author uses the Arnold-Neishtadt KAM theorem, suitable for systems with proper degeneracy, when the unperturbed Hamiltonian depends on some, but not on all the actions and the perturbation removes the degeneracy (see [2], Chap. 5)-, [32], and [35].

Bifurcation of periodic orbits. The presence of two families of elliptic periodic orbits, around the origin, for $\mu<0$ follows from the Liapunov center theorem. Moreover, for $\mu=0$, the two families still persist and hold the equilibrium point $\widetilde{O}$ while, for $\mu>0$ the two families become one unique family of elliptic periodic orbits that detaches from the origin when $A>0$ (when $A<0$ the family no longer exists for $\mu>0$ ). This last assertion follows from the Theorem 1 below (versioned from [36] and [34]). Fig. 1 (see [34], Chap. 11) shows the evolution of the families of periodic orbits with respect to the parameter $\mu$ in $(Q, T)$ variables, being $Q=\sqrt{\tilde{x}_{1}^{2}+\tilde{x}_{2}^{2}}$ the amplitude of the periodic orbit, which is constant in these variables, and $T$ the period.

Theorem 1. If $A>0$ then there exists a neighborhood $\mathcal{N}$ of $\widetilde{O}$, a $\mu_{0}>0$, an $h_{0}>0$, and a two parameter family of periodic solutions of the Hamiltonian (29), denoted by $\phi(t, \mu, h)$, which lie in $\mathcal{N}$ for all $|\mu|<\mu_{0},|h|<h_{0}, h \neq 0$ when $\mu \leq 0$. The parameter $h$ may be taken as the value of the Hamiltonian. The function $\phi(t, \mu, h)$ is real analytic in all its arguments in its domain of definition. For all values of $\mu$ under consideration the periodic solution $\phi(t, \mu, h)$ is elliptic. For $\tilde{\mu}$ fixed and $-\mu_{0} \leq \tilde{\mu} \leq 0, \phi(t, \tilde{\mu}, h) \rightarrow 0$ as $h \rightarrow 0^{ \pm}$and the frequency tends to $\varpi \pm \sqrt{\varepsilon(\tilde{\mu})}+\mathcal{O}(\tilde{\mu})$. For $\tilde{\mu}$ fixed and $0<\tilde{\mu} \leq \mu_{0}$, the one parameter family of periodic solutions $\phi(t, \tilde{\mu}, h)$ does not contain the equilibrium $\widetilde{O}$.

The case $A<0$ is also discussed in the quoted references. For a thorough study of the Hamiltonian Hopf Bifurcation the reader is pointed to [56].

(Quasi) periodic bifurcation. In the literature (see [8]), the bifurcating phenomena described in Theorem 1 are referred to as the supercritical Hopf bifurcation. Thus, the system defined by Hamiltonian (29) undergoes this bifurcation at the equilibrium $\widetilde{O}$, and, consequently, so does the system defined by the initial Hamiltonian (5) at $L_{2}$. Thus, all the families of periodic orbits that evolve and detach are elliptic and it is known that, when the elliptic directions are present in the monodromy of a periodic orbit then, generically, a Cantor family of quasi-periodic solutions appear "wrapping" that periodic orbit. One way to see this is singling out an elliptic periodic orbit and take normal form around it (see [11], [12]), then the unfolding of 2D Lagrangian invariant tori follows as a particular case from the results exposed in [8], [26] and [29].

As more than two degrees of freedom are considered, families of elliptic lower dimensional reducible tori (typically KAM tori) can undergo collisions of characteristic multipliers on the unit circle. Under suitable conditions of non resonance and 


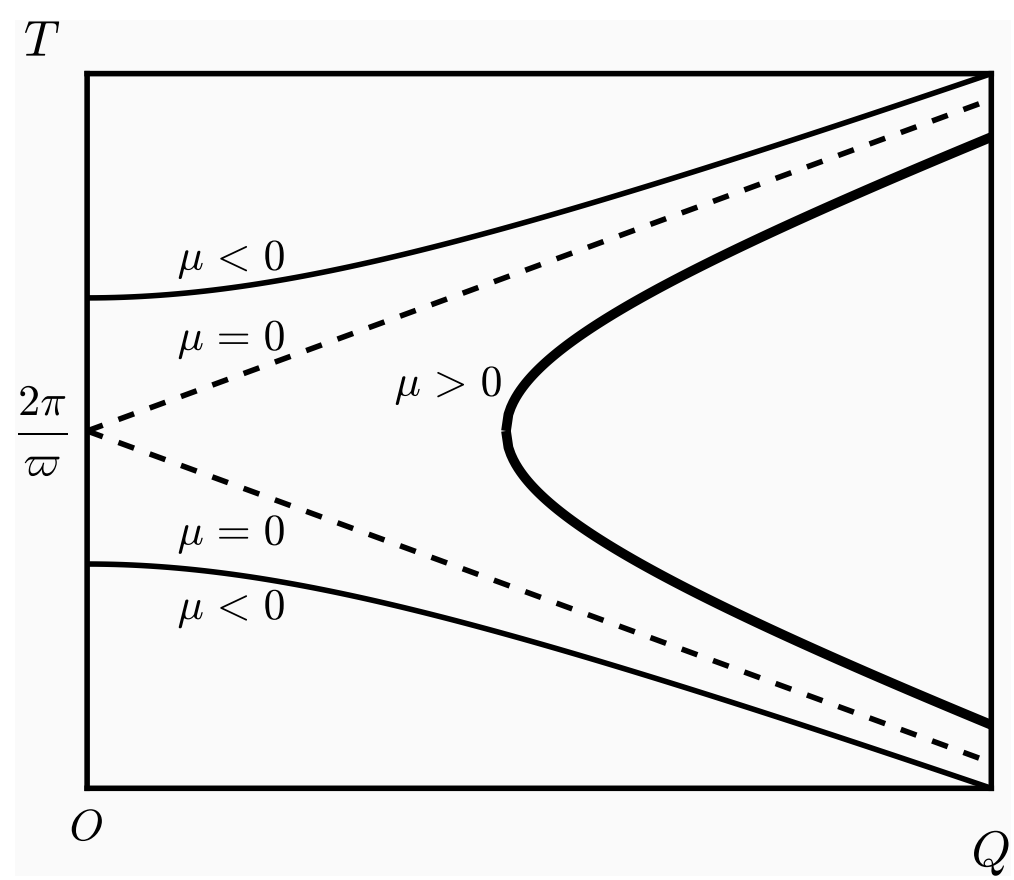

Figure 1: The bifurcated families of periodic orbits. In the horizontal axis is represented the amplitude $Q$ of the orbit and in the vertical axis the period $T$. Continuous line corresponds to the Liapunov families for $\mu<0$. The dashed line represent the two families for $\mu=0$, and the detached family for $\mu>0$ is plotted in thicker line.

non degeneracy, it gives rise to the unfolding of families of tori of higher dimension. For an account of these phenomena, known globally as quasiperiodic Hopf bifurcation, see [8].

The next proposition follows from the normal form (31), that is gleaned from the computations made at Examples 3 and 4 in Appendix AppendixD, the fact that the computed value of the coefficient $A$ is positive (see (32)), and then from the application of the inverse change (30), the Theorem 1, and the results in [11], [12], [26], [32], [54]. It describes the local behavior of the trajectories traced by the Rydberg's electron in a neighborhood in the phase space $\left(x, y, p_{x}, p_{y}\right)$ of the equilibrium point $L_{2}$, for values of the polarization parameter, $K$, close to $K_{\text {crit }}$.

Proposition 3. The equilibrium point $L_{2}$ of Hamiltonian (5) is stable for $K \leq K_{\text {crit }}$ and unstable for $K>K_{\text {crit }}$. For $K<K_{\text {crit }}$ there exist two Liapunov families of elliptic periodic orbits that contain the equilibrium point. Both families still persist for $K=K_{\text {crit }}$. For $K>K_{\text {crit }}$ the two families become one unique family of elliptic periodic orbits that no longer holds the equilibrium. Moreover, if for some $K$ close to $K_{\text {crit }}$ one singles out an elliptic periodic orbit, then the excitations in the elliptic directions yield the unfolding of a Cantor family of Lagrangian 2D-tori having that periodic orbit as its fiber.

This justifies that the normal form $\widetilde{Z}^{(s)}$ can be used to approximate the dynamics of the Hamiltonian (29) close to the equilibrium $\widetilde{O}$, and so, to explore the local dynamics of Hamiltonian (5) in a neighborhood of $L_{2}$.

\section{Dynamics of the Hamiltonian normal form}

In this section we describe in detail the dynamics close to the Hopf bifurcation and the bifurcated invariant objects involved when $\mu$ is close to 0 in Hamiltonian (29), that is, when $K$ is near $K_{\text {crit }}$ in the original Hamiltonian (5). For this purpose it is useful to consider the polar coordinates $Q, \theta$ and their canonically-conjugated momentum and action $P$ and $J$ (see [23]),

$$
\begin{array}{ll}
\tilde{x}_{1}=Q \cos \theta, & \tilde{y}_{1}=P \cos \theta-\frac{J}{Q} \sin \theta, \\
\tilde{x}_{2}=-Q \sin \theta, & \tilde{y}_{2}=-P \sin \theta-\frac{J}{Q} \cos \theta .
\end{array}
$$

In the new symplectic coordinates $(\theta, Q, J, P)$, the normal form (31) becomes an (integrable) Hamiltonian, $\Gamma$, with real coefficients

$$
\Gamma(\mu, Q, J, P)=\Gamma_{0}(\mu)+\omega(\mu) J+C J^{2}+\frac{P^{2}}{2}+\frac{J^{2}}{2 Q^{2}}+\left(B J-\frac{\varepsilon(\mu)}{2}\right) Q^{2}+A Q^{4},
$$

with

$$
\Gamma_{0}(\mu)=\tilde{\alpha} \mu+\tilde{\beta} \mu^{2}
$$

and the coefficients $\tilde{\alpha}, \tilde{\beta}, \omega(\mu), \varepsilon(\mu), A, B$, and $C$ those in (32). As $\Gamma(\mu, Q, J, P)$ does not depend explicitly on the angle $\theta, J$ is a first integral of (35), so $\Gamma(\mu, Q, J, P)$ can be regarded as a 1-degree of freedom Hamiltonian depending on the coordinates 


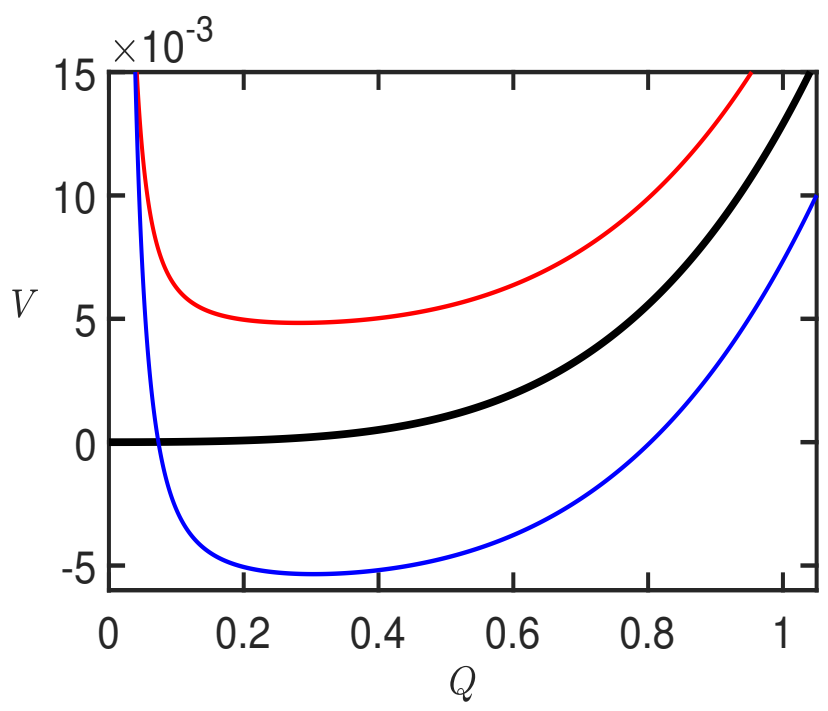

(a)

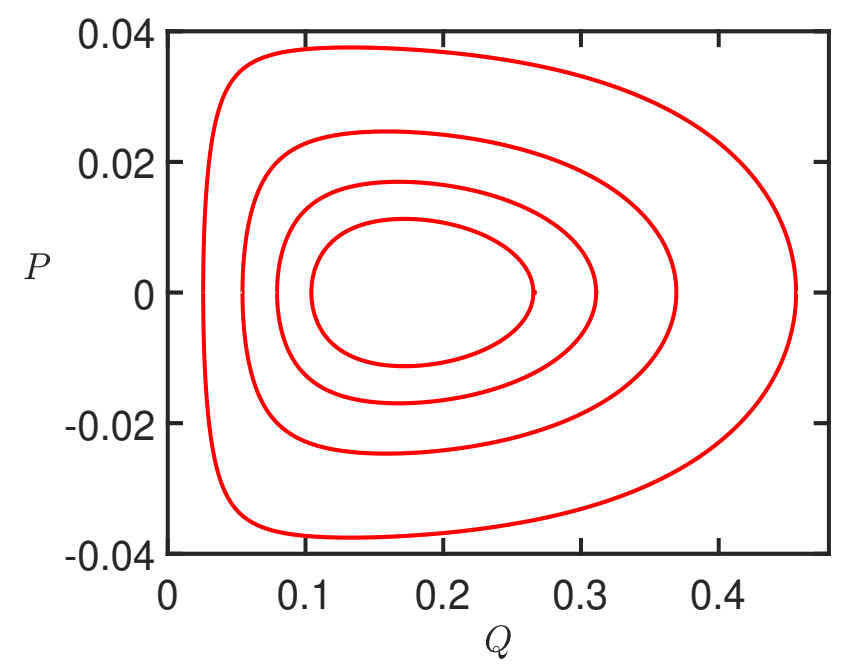

(b)

Figure 2: $\mu=-0.001<0$. (a) Potential curves $(Q, V(\mu, Q, J)), J=0$ (black thick line), $J>0$ (red line), $J<0$ (blue). (b) $(Q, P)$ variables and $E=0.003$. From outer to inner curves, $J=0.001,0.0015,0.0017,0.0018$.

$(Q, P)$, whereas $J$ (and $\mu$ ) will be thought of as parameters. Thus, the difference $\Gamma-\Gamma_{0}(\mu)$ is a natural Hamiltonian, made up of the sum of kinetic plus potential energy, i.e., $\Gamma-\Gamma_{0}(\mu)=P^{2} / 2+V(\mu, Q, J) / 2$, being

$$
V(\mu, Q, J):=2 \omega(\mu) J+2 C J^{2}+\frac{J^{2}}{Q^{2}}+(2 B J-\varepsilon(\mu)) Q^{2}+2 A Q^{4} .
$$

To obtain the phase portraits for different sets of parameters, an easy way to proceed is to obtain the relation

$$
P= \pm \sqrt{E-V(\mu, Q, J)}
$$

with $E=2\left(\Gamma-\Gamma_{0}(\mu)\right)$ and we simply consider the potential curves $(Q, V(\mu, Q, J))$ for a given value of $\mu$. We plot, in Figs. 2(a) and 3(a), the potential curves for $\mu=-0.001<0$ (similar for $\mu=0$ ) and $\mu=0.001>0$ and taking different values of $J$.

We recall that, for any value of $\mu$ fixed, we have an equilibrium point which is the origin $-L_{2}$ in original variables- for $J=0$ and $E=0$, i.e. $\Gamma=\Gamma_{0}(\mu)$. Now, let us focus on each of the three cases $\mu<0, \mu=0$ and $\mu>0$ and describe the dynamics in $(Q, P)$ variables, when varying $E$.

- If $\mu<0$ is fixed and small, the origin is a center $\times$ center equilibrium point with characteristic exponents $\pm i \omega_{1}$ and $\pm \mathrm{i} \omega_{2}$ (depending on $\mu$ ).

- For $E=0$, we have just the equilibrium point and 2D invariant tori -varying $J<0$-.

- For a fixed $E>0(E<0)$, we have a stable periodic orbit -associated with the minimum point of the potential $V(\mu, Q, J)$ for a suitable value of $J$-surrounded by $2 \mathrm{D}$ invariant tori (corresponding to different values of $J$ ). In the phase portrait in $(Q, P)$ variables, they can bee seen respectively as a fixed point (on the $Q$ axis) surrounded by closed curves. See Fig. 2.

- Varying $E>0$ (similarly $E<0$ ), we obtain a family of periodic orbits -the minimum points of $V(\mu, Q, J)$ when varying $J>0(J<0)$ - which are stable. So we obtain two families of periodic orbits, parameterized by the energy, that are born at the equilibrium point. This is in accordance with the Liapunov center Theorem.

- If $\mu=0$, the origin is a degenerated center $\times$ center equilibrium point with characteristic exponents $\pm i \omega_{1}= \pm i \omega_{2}=$ $\pm i \omega$. Then the two families of periodic orbits, parametrized by the energy $E$, become one family in the sense that when the energy $E$ tends to 0 , the characteristic multipliers of the periodic orbit tend to the same value $2 \pi / \omega$. As in the previous case, for a fixed $E>0(E<0)$, we have a periodic stable orbit surrounded by $2 \mathrm{D}$ invariant tori. See Fig. 2 (the qualitative plot for $(Q, V(\mu, Q, J))$ is the same for both $\mu<0$ and $\mu=0)$.

- If $\mu>0$, the origin is a complex saddle equilibrium point with characteristic exponents $\pm a_{1} \pm \mathrm{i} b_{1}$. The typical behavior for a direct Hopf bifurcation can be shown here.

- A main difference with respect to the two previous cases $\mu \geq 0$, is that, for $E=0$, there exist 2D invariant unstable and stable manifolds associated with the equilibrium point, $W^{u}(0)$ and $W^{s}(0)$, which are coincident 
(they will not be so in original coordinates, of course). Their parametrization is obtained from the level sets $E=0$ and $J=0$ and (36),

$$
W^{u, s}(\widetilde{O})=\left\{(\theta, Q, J, P)=(\theta, Q, 0, \pm \sqrt{-V(\mu, Q, 0)}), \theta \in \mathbb{T}^{1}, 0<Q \leq \sqrt{\varepsilon(\mu) /(2 A)}\right\}
$$

where the plus sign describes the unstable (outgoing) manifold whereas the minus sign describes the stable (incoming) one (see Fig. 3(b)). Of course, more precise parametrizations can be derived taking normal form up to a convenient high degree. See [40] for a more detailed account on parametrizations of invariant objects in a similar context.

- As expected in a supercritical Hopf bifurcation the family of periodic orbits that bifurcates is detached from the origin (the equilibrium point). This can be easily seen from the potential curves $(Q, V(\mu, Q, J))$ for $E=0$, where the periodic orbit (a minimum of the potential curve for a suitable $J$ value) has a $Q>0$ value (see Fig. 3(a)). We can follow this family of periodic orbits varying the energy $E$.

- These bifurcated periodic orbits that appear on the complex unstable side are stable, so they are surrounded by $2 \mathrm{D}$ invariant tori.

- For $E=0$, we can distinguish between two kinds of 2D invariant tori: the internal ones that are born around the bifurcated periodic orbit and the external KAM tori that already existed for $\mu<0$ (see Fig. 3(b)).

A final important comment is that we have described the dynamics for a fixed value of $\mu>0$ and $\mu<0$ of $\Gamma$ in (35) which is an integrable Hamiltonian. Of course this normal form Hamiltonian is just an approximation of the original one, which is non integrable. So the "integrable" part of the dynamics described so far is valid only as an approximation of the real dynamics of the original Hamiltonian for a local neighborhood both of the equilibrium point (the origin in $(Q, P)$ variables and $L_{2}$ in original ones) and values of $\mu$ close to 0 (for Hamiltonian (35)), that is, values of $K$ near $K_{\text {crit }}$.

The next step is to describe the dynamics of the original Hamiltonian and the consequences of the Hopf bifurcation. Our approach is to consider not only a neighborhood of the equilibrium point but a large region of the phase space configuration. We will take values of $K$ in an interval (not necessarily small) containing $K_{\text {crit }}$. We will also show that the periodic stable orbits and the invariant manifolds (of $L_{2}$ and of the Liapunov periodic orbits) are invariant objects that become relevant for the dynamics.

Before focusing on the original Hamiltonian, and in order to have a self contained paper, we include a short Section devoted to the numerical methodology used to compute such relevant invariant objects.

\section{Numerical computation of the invariant objects involved}

We will be focused on Poincaré section plots (PSP), equilibrium points, periodic orbits and their manifolds.

\subsection{Poincaré section plots (PSP)}

For a given $K$ and $H=h$, being $H$ the original Hamiltonian (5), we consider the original system of ODE (6) and the Poincaré section $\Sigma: x^{\prime}=p_{x}+y=0$. We will call the Poincaré section plot (PSP) the plot obtained with the intersection of a given solution -or many solutions- (in this level of energy $h$ and integrated for a range of time) with $\Sigma$ such that $y^{\prime}=p_{y}-x<0$. Actually the PSP will be the plot with the $(x, y)$ projection of such intersection points.

\subsection{Equilibrium points and $2 D$ associated manifolds}

Once $K$ is given, in order to find the equilibrium points, we simply solve the polynomial equation of third degree (14). So $L_{i}$ is obtained with coordinates $\left(x_{L_{i}}, 0,0, x_{L_{i}}\right)$, for $i=1,2$.

Next, for $K>K_{\text {crit }}$, in order to compute the 2D unstable/stable manifold of $L_{2}, W^{u / s}\left(L_{2}\right)$, we will take a set of initial conditions on it. For each initial condition we will compute the corresponding orbit integrating the system of ODE -forward in time for $W^{u}\left(L_{2}\right)$ and backwards in time for $W^{s}\left(L_{2}\right)$ - and checking that the Hamiltonian value $h\left(L_{2}\right)$ remains constant along the integration for all the range of time considered.

To find such a set of initial conditions we can proceed following two different approaches:

(i) Either we take the set of initial conditions from the fourth order normal form, that is, for $\mu>0$ small given, we consider $(Q, P, \theta, J)$ with $(Q, P)$ in a neighborhood of the origin and satisfying (36), $\theta \in[0,2 \pi], \mu>0$ small and $J=0$ (see Fig. 3), we apply the normalizing change of variables that reduces the Hamiltonian (5) to the form (29) -see Remark AppendixD.8-, we obtain a value of $K$ and an approximation of the initial conditions in the original variables $\left(x, y, p_{x}, p_{y}\right)$ of Hamiltonian (5).

(ii) Or we take a set of initial conditions, using directly the original variables, on the linear approximation of the manifold, that is on its tangent plane. More precisely: we consider the Jacobian matrix of system of ODE (6), its eigenvalues $\lambda_{1,2}=a_{1} \pm \mathrm{i} b_{1}, \lambda_{3,4}=-a_{1} \pm \mathrm{i} b_{1}$ with $a>0$. Let us denote $\boldsymbol{u}_{1} \pm \mathrm{i} \boldsymbol{u}_{2}$ and $\boldsymbol{u}_{3} \pm \mathrm{i} \boldsymbol{u}_{4}$ the associated eigenvectors. To compute the unstable manifold $W^{u}\left(L_{2}\right)$, we take the orthonormal basis $\boldsymbol{v}_{1}, \boldsymbol{v}_{2}$ (obtained from $\boldsymbol{u}_{1}, \boldsymbol{u}_{2}$ and generating the same plane through $L_{2}$ ) and a circle (centered at $L_{2}$ and radius $\xi>0$ small, typically $\xi=10^{-6}$ ) of initial conditions given by

$$
L_{2}+\xi\left(\cos s \boldsymbol{v}_{1}+\sin s \boldsymbol{v}_{2}\right), \quad s \in[0,2 \pi]
$$

For the computation of the stable manifold $W^{s}\left(L_{2}\right)$, we proceed similarly taking into account $\boldsymbol{u}_{3}$ and $\boldsymbol{u}_{4}$. 


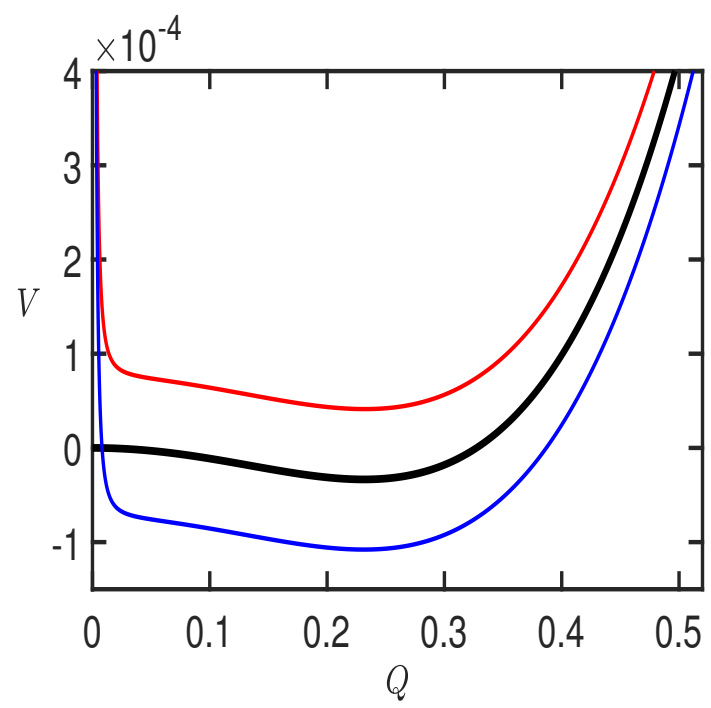

(a)

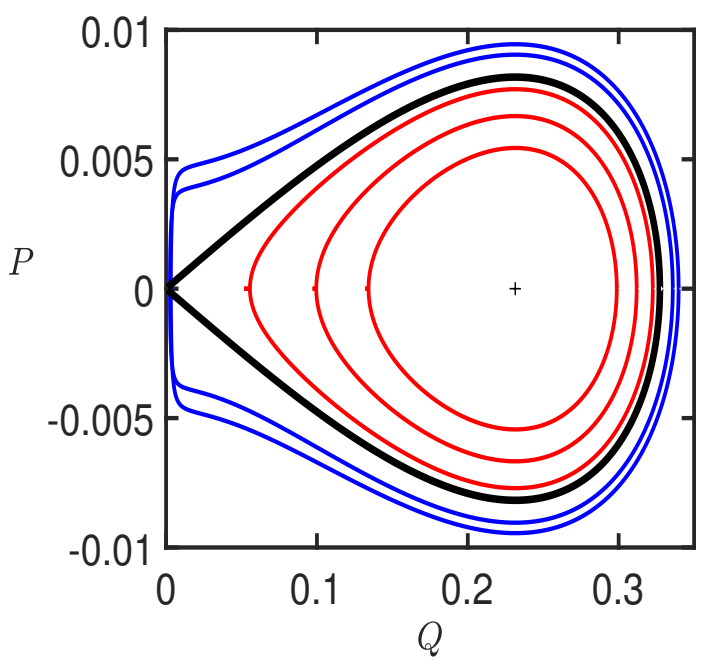

(b)

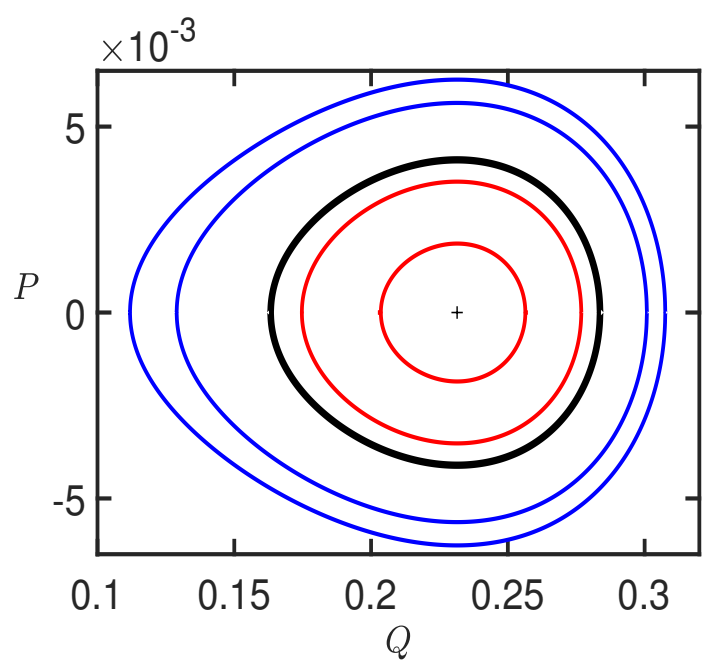

(c)

Figure 3: $\mu=0.001>0$. (a) $(Q, V(\mu, Q, J))$ for three values of $J: J=0.0001$ (red), $J=0$ (thick black line), $J=-0.0001$ (blue). (b) $(Q, P)$ coordinates at the level set $E=0$. The 2D Lagrangian tori with $J<0$ are drawn in blue. For $J=0$, the thick black line represents the pinched torus, i.e., the union of the equilibrium point with its stable and unstable manifolds (note that these manifold coincide, for the normal form is integrable) and, in red, the 2D Lagrangian tori around the detached bifurcated elliptic periodic orbit with $J>0$ ). (c) Same line color code for $E=-0.00005$.

We have implemented both methods achieving the same results. Of course, the first approach can be applied only for values of $K$ very close to $K_{\text {crit }}$ (i.e. $\mu>0$ small), whereas the second one works for any $K$.

\subsection{Periodic orbits, stability and $2 D$ associated manifolds}

To compute families of periodic orbits (PO), a standard methodology consists in solving the nonlinear system of equations (using a predictor-corrector method)

$$
\begin{aligned}
H(z)-h & =0, \\
g(z) & =0, \\
\Phi_{T}^{H}(z)-z & =0,
\end{aligned}
$$

where $\Phi_{t}^{H}(z)$ is the time- $t$ flow of the ODE system (6), $z$ is an initial condition of the PO, $T$ is its period $\left(\Phi_{T}^{H}(z)=z\right)$ and $g(z)=0$ is a suitable Poincaré section (a hypersurface in $\mathbb{R}^{4}$ ).

To do so we need a seed for the (approximation of the) initial condition of a PO. Once we have one PO we proceed with the continuation of a family.

Since we will focus mainly on the bifurcated periodic orbits with $H=h\left(L_{2}\right)$ (it is the value of $h$ where the manifolds $W^{u, s}\left(L_{2}\right)$ exist), varying $K>K_{\text {crit }}$, to get a seed for an initial condition of a PO, we can proceed again in two different ways: 


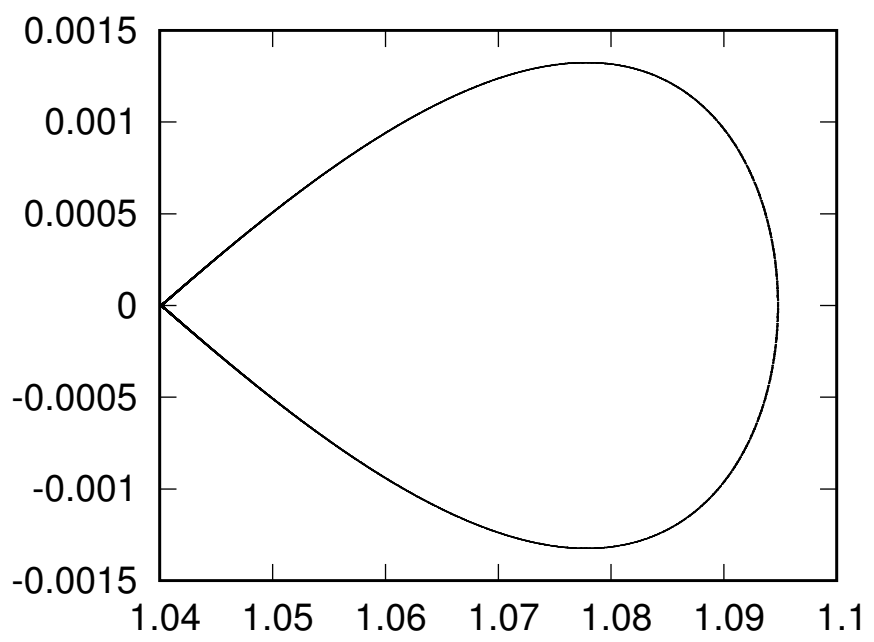

(a)

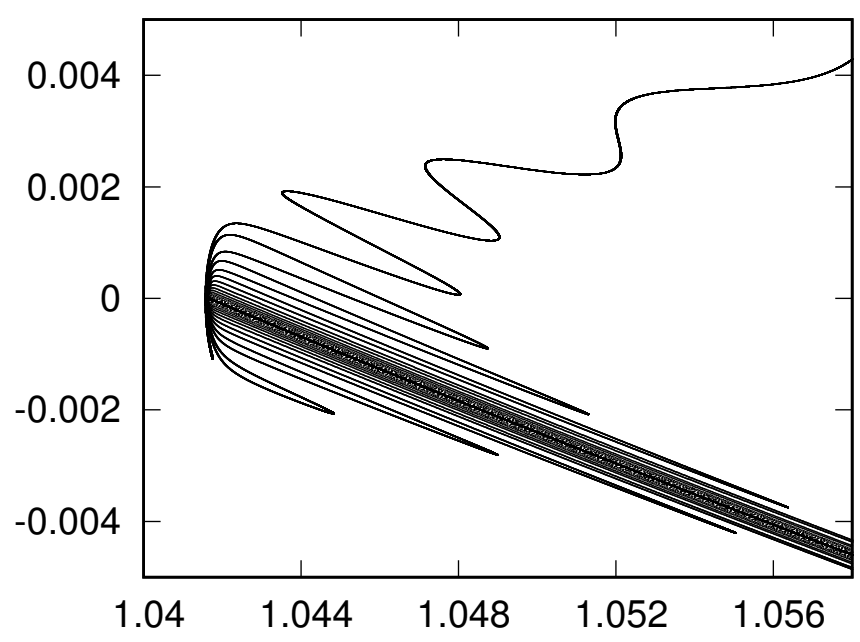

(c)

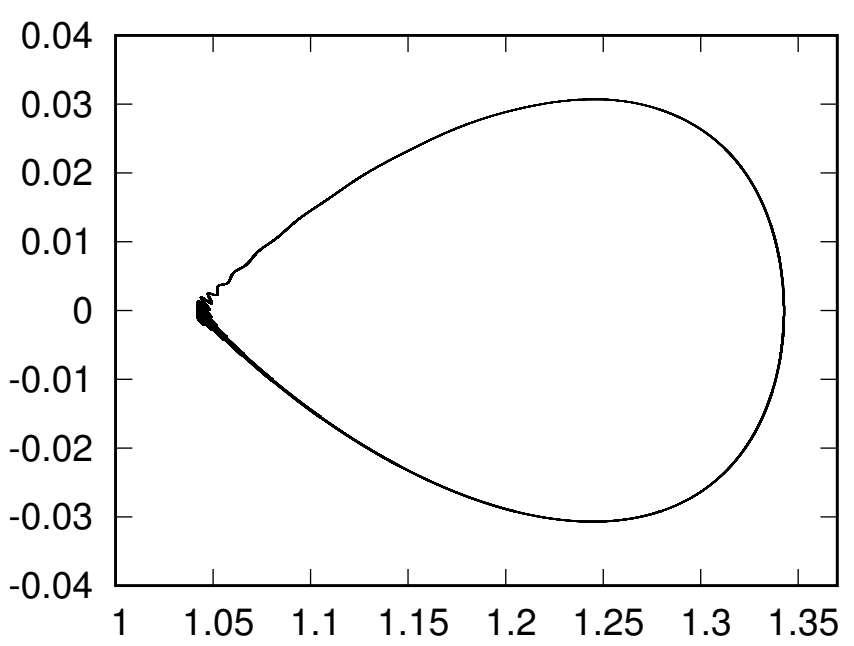

(b)

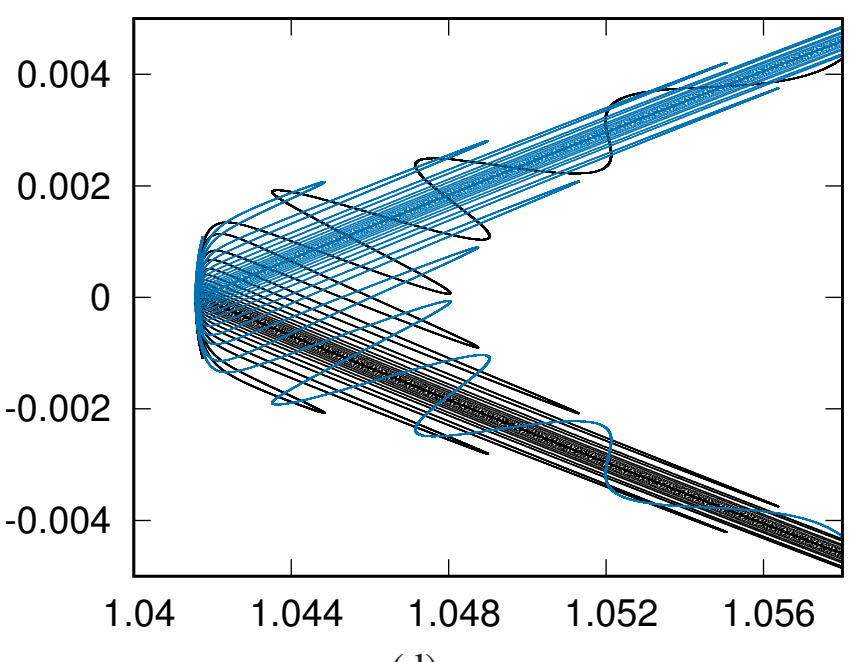

(d)

Figure 4: $(x, y)$ projection of the PSP for $W^{u}\left(L_{2}\right), W^{s}\left(L_{2}\right)$. (a) $K=0.1157$. (b) $K=0.12$, just $W^{u}\left(L_{2}\right)$. (c) A zoom close to $L_{2}$. (d) A zoom with both $W^{u}\left(L_{2}\right)$ and $W^{s}\left(L_{2}\right)$.

(i) Either we take the initial conditions from the fourth order normal form, that is, for $\mu>0$ small given, we consider $\left(Q, P, \theta_{2}, J\right)$ with $P=0, E=0$ in $(36), \theta=0$, and $(Q, J)$ is a minimum of $V(\mu, \theta, J)$ i.e., a solution of the system

$$
V(\mu, Q, J)=0, \quad \frac{\partial V}{\partial Q}(\mu, Q, J)=0
$$

(see Fig. 3), we apply the composition of the successive changes of coordinates that lead to the normal form (as above), we obtain a value of $K$ and the corresponding (approximation of the) initial conditions on the original variables $\left(x, y, p_{x}, p_{y}\right)$ of the PO.

(ii) Or, we fix $K>K_{\text {crit }}$ and $H=h\left(L_{2}\right)$. We consider a set of different initial conditions of type $\left(x, 0,0, p_{y}\right)$, with $y^{\prime}=p_{y}-x<0$, varying $x$ ( $y^{\prime}$ is obtained from the Hamiltonian) in a suitable interval; for each initial condition we integrate the original system (6) and obtain the corresponding solution for a big enough range of time, and we save just the points belonging to the Poincaré section plot (PSP). In the PSP obtained (see for example Fig. 6(b)), we zoom the area around the bifurcated stable PO (surrounded by invariant curves) and we have an approximation of $x$ (and $p_{y}$ ) of the initial condition of the required PO.

We have implemented both strategies to get a seed and both have become satisfactory -again the first one only valid for $K$ very close (and bigger than) to $K_{c r i t^{-}}$. We have implemented Newton's method for the correction to solve system (38) to refine the seed and obtain the bifurcated PO for $H=h\left(L_{2}\right)$ when varying $K>K_{\text {crit }}$ (see next Section). The absolute tolerance to stop Newton iterates in the solution has been typically $10^{-11}$.

Given a PO, in order to determine its (linear) stability, we have integrated both the system of ODE (6) together with its first variational equations to obtain the monodromy matrix $M$ after a period. We recall that the value of the stability parameter $k=\operatorname{Tr} M-2$ decides the stability character of a PO: it is stable (elliptic) if $|k|<2$, and unstable (hyperbolic) if $k \in \mathbb{R} \backslash[-2,2]$ (see for example [9] for details). 
As will be shown in the next Section, the $2 \mathrm{D}$ invariant manifolds of the Liapunov orbits around $L_{1}, o l_{1}$, will also play a role on the description of the global dynamics. Such orbits (for the values of $K$ and $h$ considered) are unstable (the monodromy matrix has as eigenvalues $1,1, \lambda>1$ and $1 / \lambda<1$, i. e. $k \in \mathbb{R} \backslash[-2,2])$. In order to generate the unstable manifold $W^{u}\left(o_{1}\right)$ such that $z_{0}$ is an initial condition of the $o l_{1}$ and $\boldsymbol{v}_{0}$ is a unitary eigenvector associated with $\lambda>1$, we consider a set of initial conditions on the linear approximation of the manifold given by

$$
\varphi(\theta)+\xi \boldsymbol{v}(\theta), \quad \theta \in[0,2 \pi]
$$

where $\xi>0$ or $<0$ to obtain the two branches that compose the manifold, small (typically $|\xi|=10^{-6}$ ) and

$$
\varphi(\theta)=\Phi_{\frac{\theta}{2 \pi} T}^{H}\left(z_{0}\right), \quad \boldsymbol{v}(\theta)=\lambda^{\theta / 2 \pi} D \Phi_{\frac{\theta}{2 \pi} T}^{H}\left(z_{0}\right) \boldsymbol{v}_{0}
$$

are a parametrization of the PO and the eigenvector of the associated monodromy matrix corresponding to the point $\varphi(\theta)$ respectively (see [4]). For each initial condition, we obtain the corresponding solution integrating forward in time system (6) and checking that the Hamiltonian remains constant along the integration. We proceed similarly to obtain the stable manifold $W^{s}\left(o_{1}\right)$ taking into account $\lambda<1$ and integrating backwards in time.

\section{Description of the dynamics of the original system}

The purpose of this Section is to describe the Hopf bifurcation effect on the dynamics of the original non-integrable Hamiltonian system for which the fourth order integrable normal form taken into account is just an approximation (and of course valid just in a tiny neighborhood of the equilibrium point $L_{2}$ and a small interval around $K_{\text {crit }}$ ). So, in the previous Sections, we considered the dynamics of the electron from a very local point of view. Now, numerical simulations applied to the original system allow to describe the dynamics in large regions in the $(x, y)$ plane and any value of $K$. We will distinguish two cases: $K>K_{\text {crit }}$ and $K<K_{\text {crit }}$ (recall that $\left.K_{\text {crit }}=3^{-4 / 3} / 2 \approx 0.11556021\right)$.

Case 1. $K>K_{\text {crit }}$. We fix the energy value $h=h\left(L_{2}\right)$ and we discuss different aspects of the dynamics when varying $K$, that can be graphically illustrated through the Poincaré section plots (PSP).

- Invariant manifolds of $L_{2}$. When $K$ is very close to $K_{\text {crit }}$, the stable and unstable 2D manifolds, $W^{u}\left(L_{2}\right)$ and $W^{s}\left(L_{2}\right)$, are almost coincident (they do coincide for the integrable normal form, but they do not for the original non integrable Hamiltonian giving rise to the splitting of separatrices). See Fig. 4(a), where the PSP of the manifolds are plotted for $K=0.1157$ very close to $K_{\text {crit }}$. As far as $K$ increases, the typical homoclinic tangle is more visible, see Fig. 4, and for bigger $K$, the manifolds become very intricate, see Fig. 6(a). We also remark that for $K$ near $K_{c r i t}$, the size of the manifolds is small, i.e. the orbits on the manifolds visit a small region in the $(x, y)$ plane. See the PSP of the manifolds in Fig. 5(c) where the manifolds are confined by external 2D tori (invariant curves on the PSP). Whereas when $K$ increases, the size of the manifolds becomes larger and there are some orbits on the manifolds that go tremendously far away from $L_{2}$ or even escape. See Fig. 6(c).

- Existence of bifurcated periodic orbits and tori. As foreseen by the normal form description, for a given $K$ bigger and close to $K_{\text {crit }}$, there exists a detached family of periodic orbits when varying $h$, in a neighborhood of $h\left(L_{2}\right)$, which are stable (for $h$ near $h\left(L_{2}\right)$ ). For the particular fixed value of $h\left(L_{2}\right)$, the corresponding bifurcated periodic orbit will be surrounded by invariant $2 \mathrm{D}$ tori, which will be located inside the region enclosed by the invariant manifolds of $L_{2}$, if $K$ is very close to $K_{\text {crit }}$, see Fig. 5(c) for $K=0.1157$ where the detached periodic orbit (a fixed point on the PSP) and the surrounding 2D tori (invariant curves) are shown. We remark, on the one hand, that the continuous family of tori inside the coinciding manifolds (Fig. 3) obtained from the normal form now becomes a Cantor family of tori (for the original Hamiltonian system) as far as $K$ is very close to $K_{\text {crit }}$. On the other hand, the destruction of many such tori is also visible when $K$ increases and the existence of chaos is much more apparent, although the stable periodic orbit persists, see Fig. 6 top for $K=0.13$.

- Confinement due to the invariant manifolds. Although the appearance of the splitting phenomenon (not present for the normal form Hamiltonian) for any $K>K_{\text {crit }}$, the manifolds somewhat enclose a confinement region, more visible as far as $K$ is close to $K_{\text {crit }}$. See Fig. 5(c). Therefore there exist points inside this region giving rise to confined solutions, the confinement size determined by the size of the manifolds. It is worth noting the different sizes of confinement regions comparing those (smaller) delimited by the invariant manifolds of $L_{2}$ and those (bigger) delimited by the 2D KAM external tori. See Fig. 5(c) for $K=0.1157$. When $K$ increases, the external tori disappear and there is a dramatic change in the intricate shape of the manifolds of $L_{2}$, see Fig. 6 top and middle for $K=0.13$.

- Confinement due to the bifurcated elliptic periodic orbits. As already mentioned, when $K$ increases, the manifolds of $L_{2}$ are very complicated and the visited region (in $(x, y)$ ) is very big. However, as far as the bifurcated periodic orbit is stable, there will be still a small region of confinement for all time, due to the existence of surrounding 2D tori. So an important effect of the Hopf bifurcated orbit is to delimit, among a chaotic sea, a region where the electron will remain confined for ever. We show this behavior on the PSP of Fig. 6(b) for $K=0.13$. 


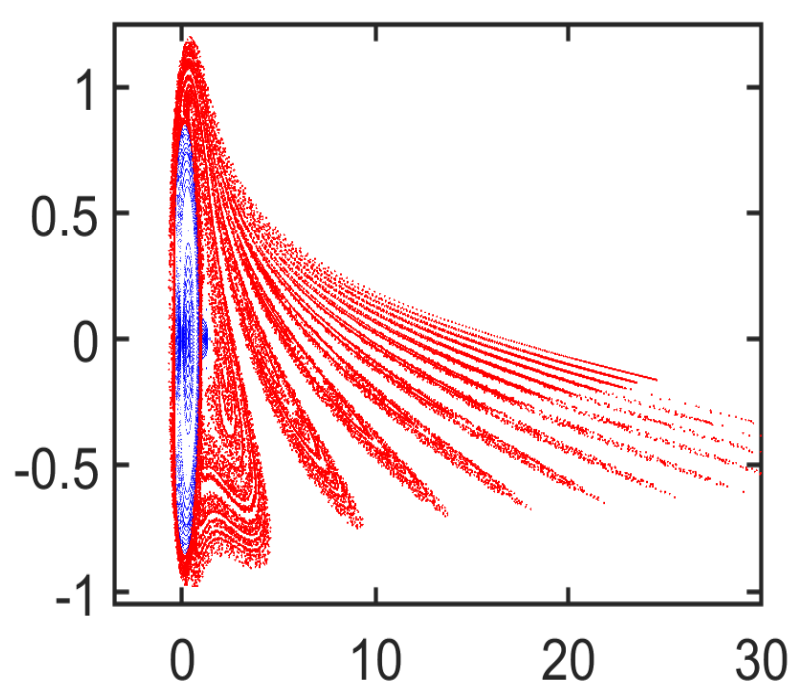

(a)

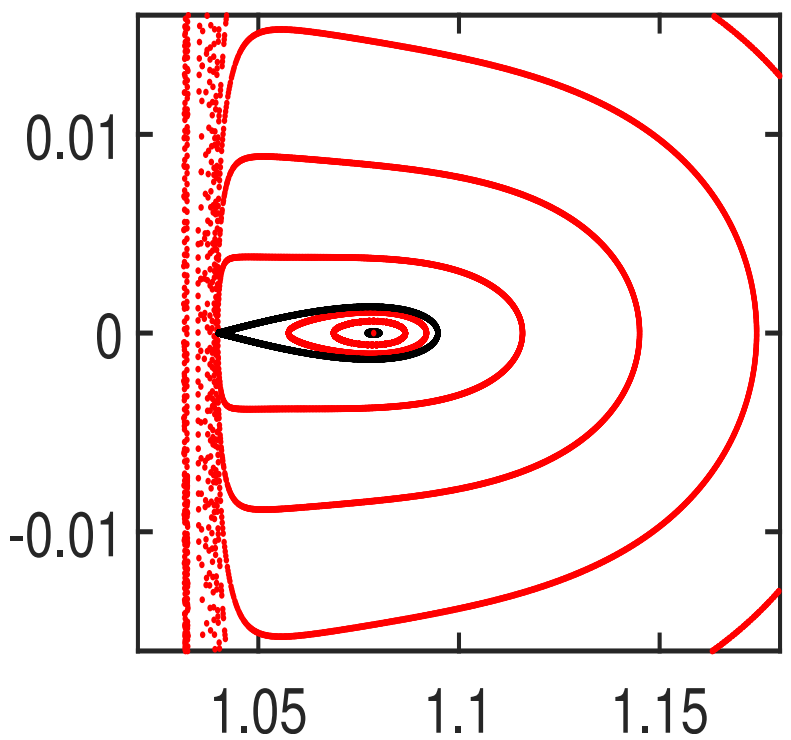

(c)

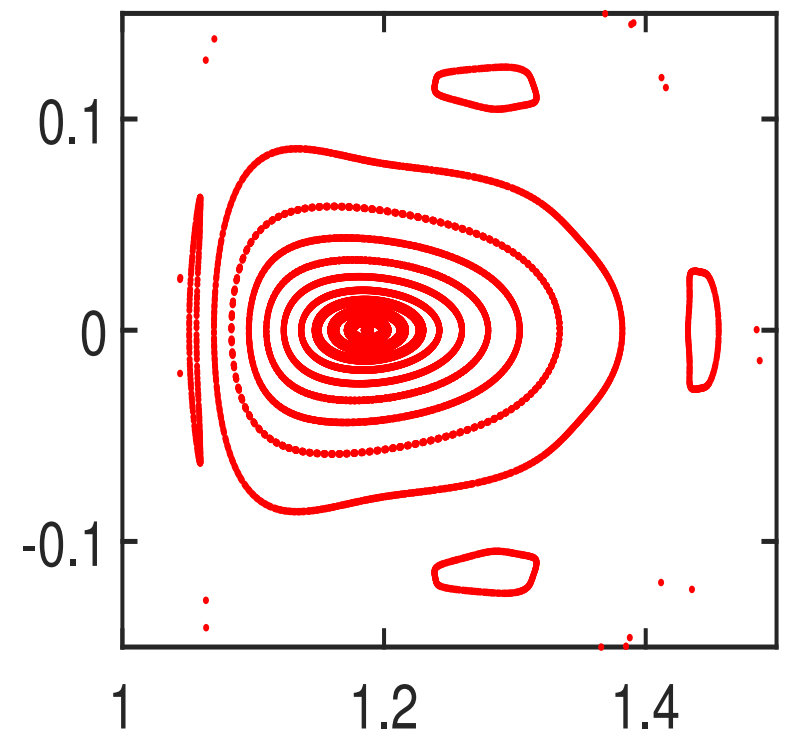

(e)

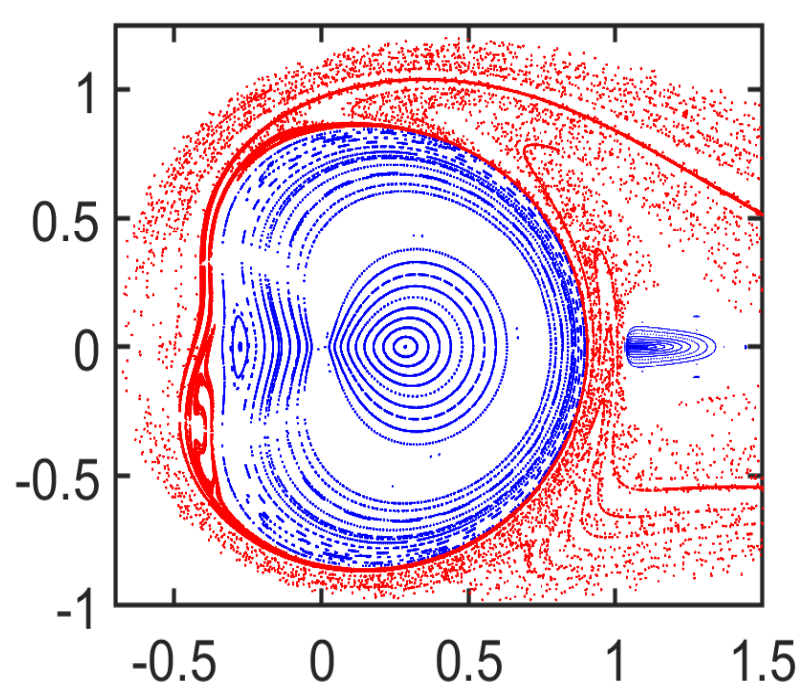

(b)

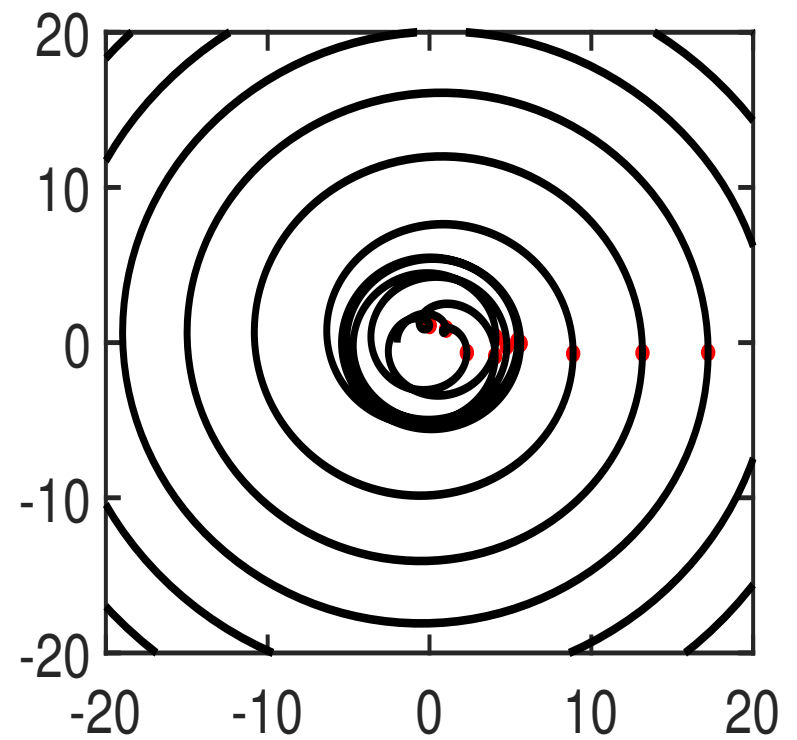

(d)

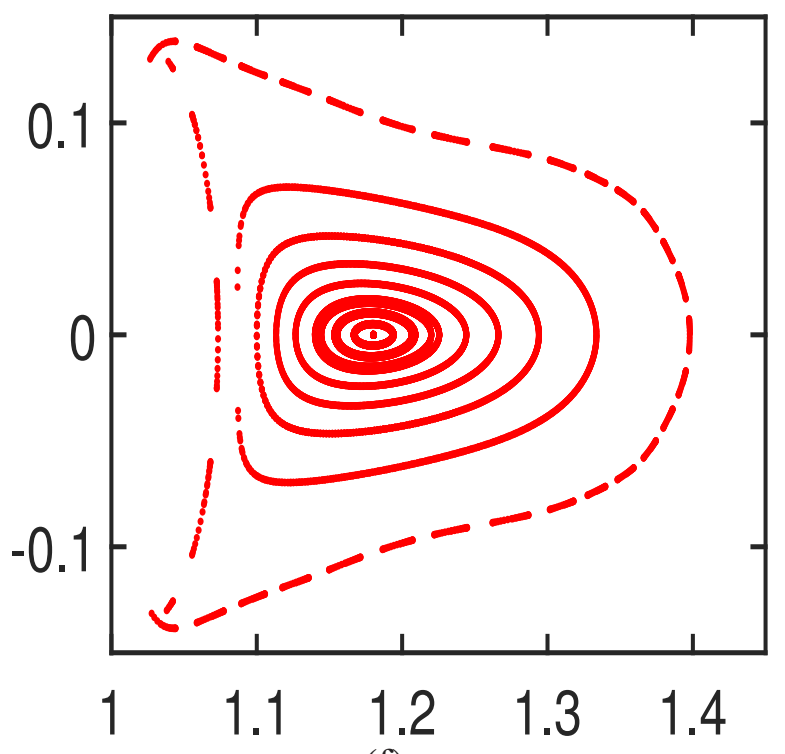

(f)

Figure 5: $K=0.1157$. $(x, y)$ projection of the PSP. Top and middle correspond to $h=h\left(L_{2}\right)$. (a) Global plot. (b) Zoom. The red points correspond to $W^{u}\left(o l_{1}\right)$; two confined regions (in blue) are clearly distinguished: the one close to the origin and the one due to the bifurcated stable periodic orbit. (c) We show the KAM invariant curves (in blue) enclosing $W^{u}\left(L_{2}\right)$ and $W^{s}\left(L_{2}\right)$ (in black), and also the bifurcated stable periodic orbit and the corresponding invariant curves surrounding it (in blue). (d) Fast escape orbit $((x, y)$ projection,in black) and the corresponding points on the PSP overlapped (in red). Bottom: 2D KAM tori around the bifurcated stable periodic orbit for $h>h\left(L_{2}\right)$ (e) and $h<h\left(L_{2}\right)$ (f). 
- Stability of the bifurcated periodic orbits. We have computed the family of bifurcated periodic orbits when varying $K$. In Fig. 7 we plot two curves on the $(K, x)$ plane: for each $K$, the $x$ value of the equilibrium point $L_{2}$ and the $x$ value of the initial condition (such that $y=x^{\prime}=0, y^{\prime}<0$ ) of the bifurcated periodic orbit for that $K$ (and $h=h\left(L_{2}\right)$ ). We can see how the family of periodic orbits is detached from the equilibrium point as far as $K$ increases from $K_{c r i t}$. The amplitude of the periodic orbits grows with $K$ and we have followed the family until the orbit passes, for some time, very close to the origin, so a regularization of the system of ODE should be required, see Fig. 7(b). Concerning their linear stability, there is also a change of stability: the elliptic orbits for $K$ close to $K_{\text {crit }}$ become unstable for bigger values of $K$. We plot in blue the stable periodic orbits and in red the unstable ones in Fig. 7(a).

- Homoclinic orbits. Erratic orbits. Due to the transversal intersections between $W^{u}\left(L_{2}\right)$ and $W^{s}\left(L_{2}\right)$, see for example Fig. 4(d), there exist infinitely many homoclinic orbits to $L_{2}$ and, as a consequence, it is well known that the variety of behaviors for the dynamics is very rich (symbolic dynamics may be applied). Nevertheless, for values of $K$ near $K_{\text {crit }}$, since the external KAM tori confine the invariant manifolds, the latter do not play any role in escape (ionization of the electron). See Fig. 5(c). But, for bigger values of $K$, the KAM tori are destroyed and there is a second effect which is the growth of the manifolds, see Fig. 6(c). Therefore, from the behavior of $W^{u}\left(L_{2}\right)$ and $W^{s}\left(L_{2}\right)$, there will coexist confined motion, some escaping orbits and some erratic "to and fro" orbits, that is, orbits that make several excursions, close to and far from $L_{2}$ (reaching different large distances), in an erratic way. Such orbits -also called multi-bump orbits- provide a mechanism of ionization; actually the electron experiences a slow ionization. In Fig. 8, some orbits on $W^{u}\left(L_{2}\right)$ are plotted to show such different behaviors; we plot $(t, r), r$ being the distance to the origin. We notice that, since for this value of $K$, the manifolds of $L_{2}, W^{u, s}\left(L_{2}\right)$ and those of the Liapunov periodic orbit $o l_{1}, W^{u, s}\left(o l_{1}\right)$, are mixed up (see Fig. 6(e)), the orbits plotted in Fig. 8, which belong to $W^{u}\left(L_{2}\right)$, have close passages to $L_{2}$ (as expected) and to $o l_{1}$ (due to the mixing), as time goes by. Some of these orbits will lead the electron to ionization. So, we see that both $W^{u, s}\left(L_{2}\right)$ and $W^{u, s}\left(o l_{1}\right)$ play a role. At this point it is worth mentioning, on the one hand, the simulations done in [10]; some plots in that paper look similar to those of Fig. 8. However, in that paper the values of $K$ considered were $K<K_{\text {crit }}$, and the role of states with medium eccentricity was discussed. On the other hand, in [5], a specific goal was to show how the periodic orbit $o l_{1}$ and its manifolds provided a mechanism for ionization (where, typically, the electron had several close passages to the periodic orbit), so, roughly speaking, only the periodic orbit (and manifolds) was taken into account. Very recently, a different mechanism of ionization, where the electron is ejected from the core and has successive excursions with passages far from and close to the nucleus is carried out (see [39]).

- Global picture. As a summary and taking into account the different invariant objects involved, we describe the dynamics of the PSP from a global point of view. We should distinguish four different regions:

- A region near the origin. A first global property to point out, and independent of the Hopf bifurcation phenomenon, is the existence of the retrograde periodic stable orbit close to the origin; this orbit together with the invariant surrounding 2D tori confine a clear region for the electron. See Figs. 5(b) and 6(e). This was already observed in [5] for very small values of $K<K_{\text {crit }}$.

- The invariant manifolds of $o l_{1}$. Regardless the value of $K$, for $h=h\left(L_{2}\right)$, there is the unstable periodic orbit around $L_{1}, o l_{1}$, and their invariant manifolds which play a clear role on the dynamics. As exhibited in [5] these manifolds visit a small $(x, y)$ region for $0<K<K_{\text {crit }}$ small, but we have just shown that they become more complex (and their homoclinic tangles as well) as $K$ grows visiting large regions in the $(x, y)$ plane, see Figs. 5 top and 6(d) and (e).

- The region influenced by the Hopf bifurcation. When $K$ is bigger and close to $K_{\text {crit }}$, and for $h=h\left(L_{2}\right)$, the dynamics of the Hopf bifurcation is very local in the sense that $W^{u}\left(L_{2}\right), W^{s}\left(L_{2}\right)$, the bifurcated stable periodic orbit and the surrounding invariant 2D tori are confined by the external KAM tori, so they do not play a significant role in the dynamics. See Fig. 5(c) for $K=0.1157$. Nevertheless, as far as $K$ increases, these KAM tori disappear and both manifolds -those of $o l_{1}$ and those of $L_{2}-$ are mixed, giving rise to many different kinds of orbits and chaos. Relevant to say (and as mentioned above) is that, among this chaos, the stable bifurcated periodic orbit and associated 2D tori, confine a clear region among this chaotic sea. See Fig. 6 (b) for $K=0.13$. However, for bigger values of $K$ such that the periodic bifurcated orbit is unstable, we obtain the same kind of PSP but there is no confinement around the periodic orbit at all.

- Fast escaping orbits. Finally and concerning the white region of the PSP (roughly speaking), if we take initial conditions in such regions we obtain fast escaping orbits, with a typical spiral behavior. See Figs. 5(d) and 6(f), where we consider $K=0.1157$ and $K=0.13$ and plot two escaping spiraling orbits and the corresponding PSP overlapped.

Let us finally remark that for $h$ close to and different from $h\left(L_{2}\right)$, the PSP are similar to the PSP for $h=h\left(L_{2}\right)$ except that the neither the equilibrium point nor the invariant manifolds exist. We show in Fig. 5(e), (f) a neighborhood of the bifurcated stable periodic orbit and the 2D tori that persist for $K=0.1157$.

Case 2. $K<K_{\text {crit }}$. This case was already studied in [5] but, for completeness, we just consider $K=0.115$ very close to $K_{\text {crit }}$ and $h$ near $h\left(L_{2}\right)$. As in Case 1, there is the big stable region close to the origin due to the retrograde $o_{r}$ orbit and 
the intricate invariant manifolds associated with the periodic orbit $o l_{1}$ that cover a big chaotic region in the $(x, y)$ plane. For $h=h\left(L_{2}\right)$, we have the equilibrium point $L_{2}$ which is stable, whereas for increasing/decreasing $h$ we obtain the corresponding family of stable Liapunov periodic orbits surrounded by $2 \mathrm{D}$ tori. This is clearly shown in Fig. 7 . Of course, this dynamics is in accordance with the theoretical results obtained in Sections 4 and 5.

\section{Conclusions}

In the current work we have explored the $\mathrm{CP}$ problem for values of the energy close to $h\left(L_{2}\right)$ and varying $K$ in an interval containing $K_{\text {crit }}$. We have distinguished two different parts: the analytical study and the numerical one.

Concerning the analytical part, since a main goal was to analyze the Hopf bifurcation appearing close to the equilibrium point $L_{2}$, a first step done was the computation of the normal form starting from the given Hamiltonian of the CP problem (not an academic theoretical Hamiltonian with a suitable expression useful to carry out the normal form, as is typically done in papers that study the Hamiltonian Hopf bifurcation). Moreover, the steps for the computation of the resonant normal form, up to any given order, have been explained in a constructive manner.

The mathematical analysis of the normal form obtained has the following consequences: (i) it allows to claim that the type of Hopf bifurcation is supercritical; (ii) the main results summarized in Proposition 3 enable us to describe the Hopf bifurcation and the local behavior of the dynamics in a neighborhood of the equilibrium $L_{2}$ for the original Hamiltonian in an interval for $K$ containing $K_{\text {crit }}$; (iii) parametrizations of the invariant objects appearing in the Hopf bifurcation (periodic orbits and stable and unstable manifolds of $L_{2}$ ) are obtained.

From the numerical point of view, such parametrizations provide good (approximate) initial conditions (refined a posteriori) of the relevant invariant objects that have been computed to analyse the Hopf bifurcation and their effect on the global dynamics. Focusing on the energy level $h=h\left(L_{2}\right)$, our conclusions, drawn from the obtained Poincaré section plots (PSP), are the following: For values of $K$ very close to $K_{\text {crit }}$, the unstable/stable manifolds of the complex saddle point $L_{2}$, $W^{u, s}\left(L_{2}\right)$, do not essentially play any role, since they cover a very tiny region in the plane $(x, y)$ and are surrounded by existing KAM 2D invariant tori that act as a barrier of motion. Roughly speaking, the electron, wherever it is, does not realize that the Hopf bifurcation has taken place. However for bigger values of $K$, the tiny and local effect becomes dramatic: the 2D surrounding tori disappear and now $W^{u, s}\left(L_{2}\right)$ are very intricate and fill a very big region of the plane $(x, y)$. Therefore, from a physical point of view, there is a rich variety of different kind of orbits for the electron to be described assuming that at some time the electron is located close to such invariant manifolds: to and fro, erratic, fast and slow ionizing orbits, are observed. Therefore a mechanism for the ionization of the electron with close passages to $L_{2}$ is obtained. Nevertheless, another conclusive effect of the Hopf bifurcation is that, due to the bifurcating stable orbits, for moderate values of $K$ bigger and close to $K_{\text {crit }}$, there is a small region of 2D tori around those periodic orbits that persist and the electron would remain for ever confined there, in the middle of a huge chaotic area.

Finally, in order to describe global behaviors of the electron for $h$ close to $h\left(L_{2}\right)$, PSP have been obtained for large regions in the configuration plane; we can conclude that, for any value of $K$ ranging from (bigger than) 0 to $K>K_{\text {crit }}$, the dynamics is mainly organized by: (i) the stable retrograde periodic orbit (around the proton) surrounded by 2D tori which compose a robust region of confinement; and (ii) the unstable/stable manifolds of the Liapunov periodic orbit $o l_{1}, W^{u, s}\left(o l_{1}\right)$, that exist for $h>h\left(L_{1}\right)$, and whose intricate homoclinic tangle, gives rise to all kind of orbits, including multi-bump, erratic and escaping orbits. These manifolds also provide a mechanism for ionization, but with several close passages to the periodic orbit, instead of $L_{2}$ ). It is worth mentioning that, for $K$ very close to $K_{\text {crit }}$, these manifolds are, by far, the responsible for ionization. However, for bigger values of $K>K_{\text {crit }}$, and $h=h\left(L_{2}\right)$, the manifolds of $L_{2}, W^{u, s}\left(L_{2}\right)$, also play a role and the both intricate $W^{u, s}\left(L_{2}\right)$, and $W^{u, s}\left(o l_{1}\right)$ get mixed altogether filling a bigger chaotic region in the $(x, y)$ plane. Therefore the mechanism of ionization -ionizing orbits with several passages to the periodic orbit and $L_{2}-$ is unquestionably present.

\section{Acknowledgments}

The authors have been supported by the Spanish MINECO/FEDER grant MTM2015-65715-P and the Catalan grant 2017SGR1049. ¿

\section{Appendix AppendixA. More notation and definitions}

Here we introduce the notation and definitions used in the appendices below. Thus, we shall denote by $\mathbb{M}_{n, m}(\mathbb{F})$ the vector space of matrices with $n$ rows and $m$ columns with coefficients in $\mathbb{F}=\mathbb{C}, \mathbb{R}, \mathbb{Z}$, or $\mathbb{N}_{0}=\mathbb{N} \cup\{0\}$. For square $n \times n$ matrices, we shall write $\mathbb{M}_{n}(\mathbb{F})$ instead of $\mathbb{M}_{n, n}(\mathbb{F})$. Thus, if $A \in \mathbb{M}_{n, m}(\mathbb{F}), A^{\top} \in \mathbb{M}_{m, n}(\mathbb{F})$ will stand for its transpose. If $u=\left(u_{1}, \ldots, u_{n}\right), v=\left(v_{1}, \ldots, v_{n}\right) \in \mathbb{F}^{n}$, then $w=(u, v) \in \mathbb{F}^{n} \times \mathbb{F}^{n}$. Furthermore, given $u=\left(u_{1}, \ldots, u_{n}\right) \in \mathbb{F}^{n}$, $I_{n}=\operatorname{diag}[1, \ldots, 1] \in \mathbb{M}_{n}(\mathbb{F})$ is the unit matrix and $J_{n}$ is the block matrix of the standard symplectic 2 -form in $\mathbb{R}^{2 n}$, i.e.,

$$
J_{n}=\left(\begin{array}{rc}
0 & I_{n} \\
-I_{n} & 0
\end{array}\right)
$$




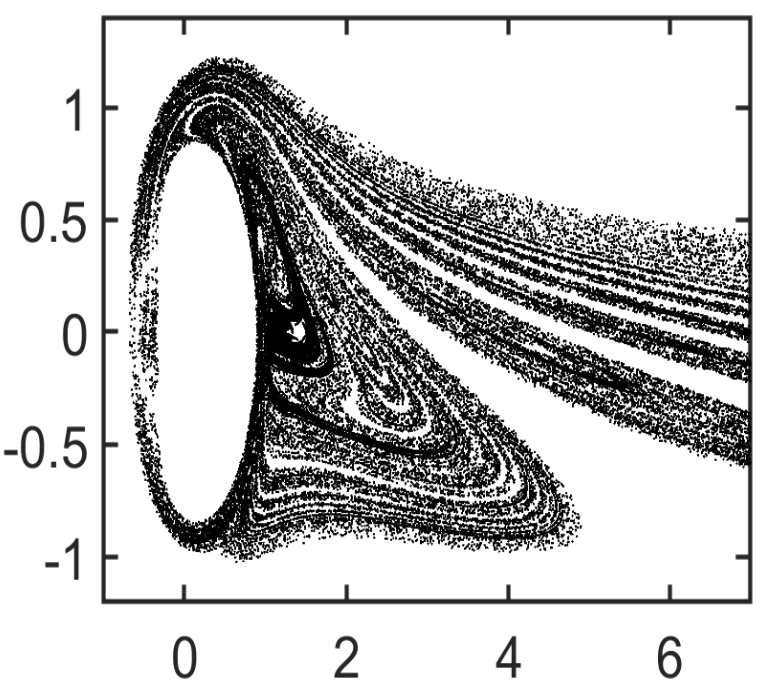

(a)

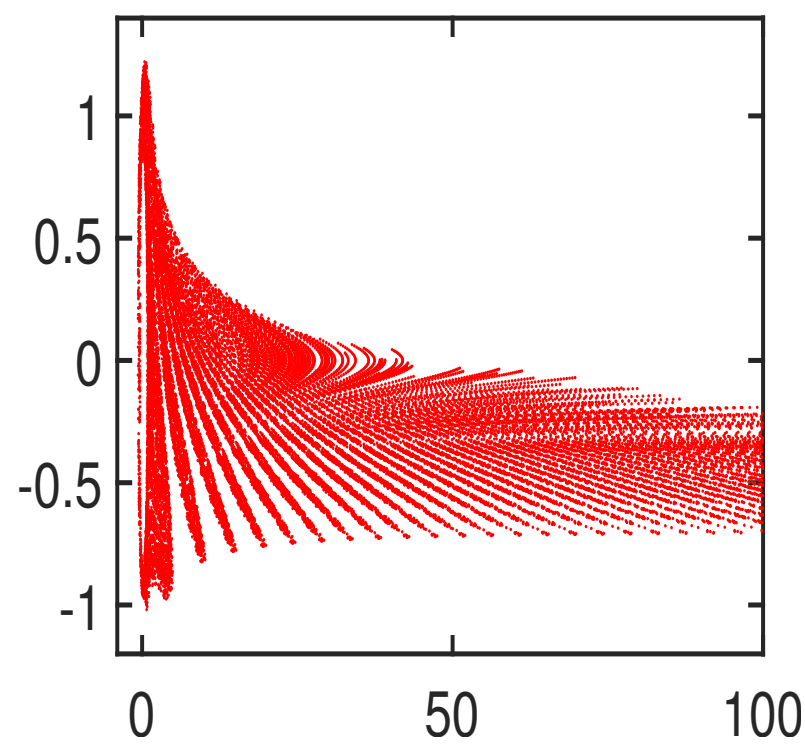

(c)

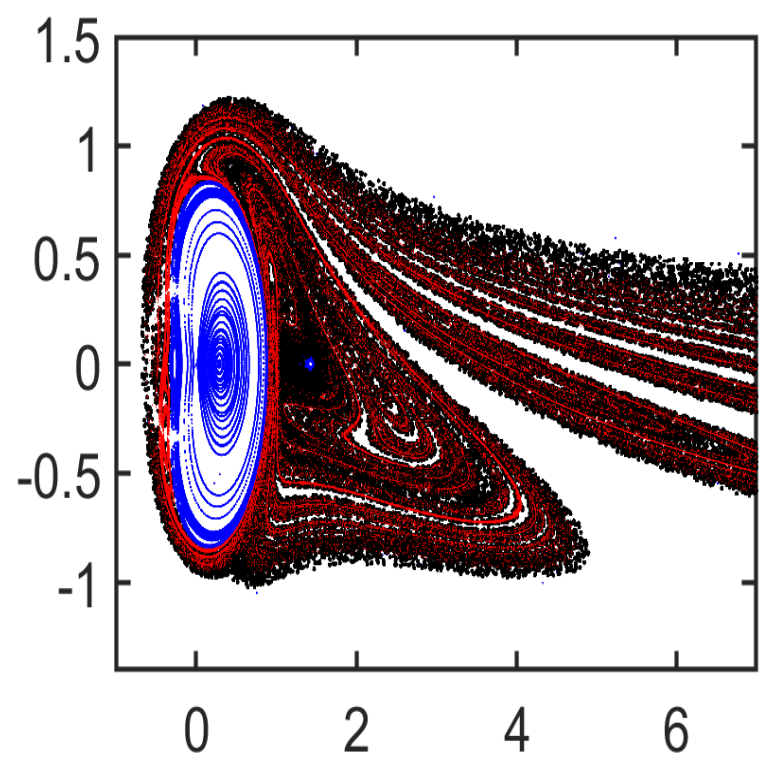

(e)

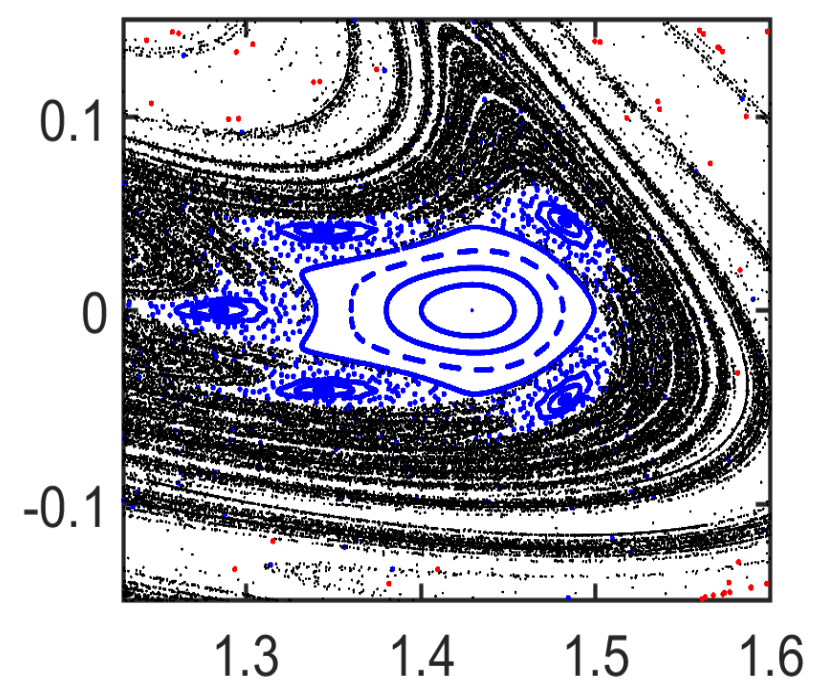

(b)

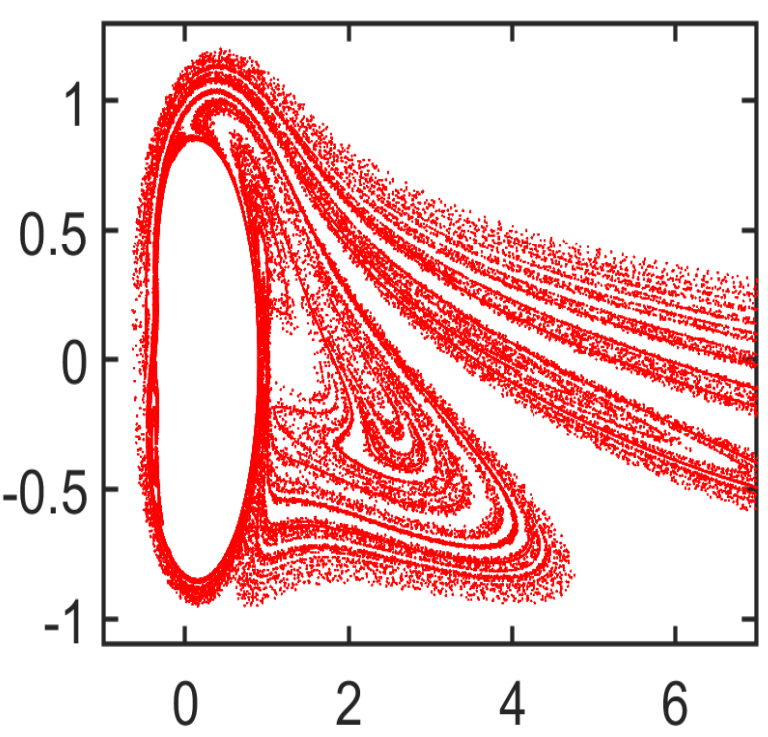

(d)

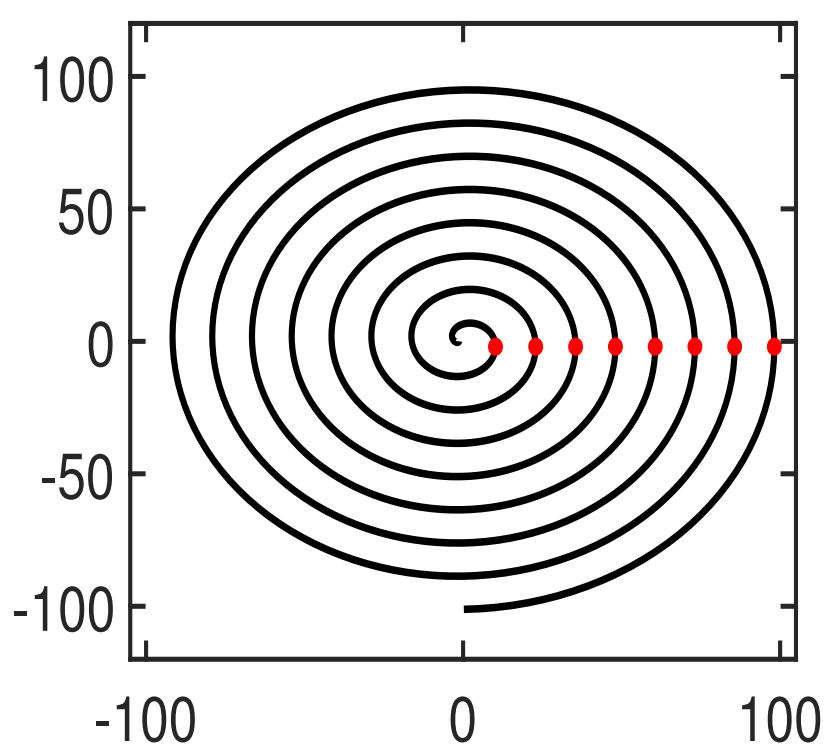

(f)

Figure 6: $K=0.13, h=h\left(L_{2}\right)$. ( $\left.x, y\right)$ projection of the PSP. (a) For $W^{u}\left(L_{2}\right)$. (b) Zoom, where the bifurcated stable fixed point and invariant curves are shown. (c) A larger window, orbits on $W^{u}\left(L_{2}\right)$ can go rather far away and even escape. (d) For $W^{u}\left(o l_{1}\right)$. (e) PSP taking into account the motion around the origin, $W^{u}\left(L_{2}\right)$ and $W^{u}\left(o l_{1}\right)$. (f) Fast escape orbit $((x, y)$ projection, in black) and the corresponding points on the PSP overlapped (in red). 


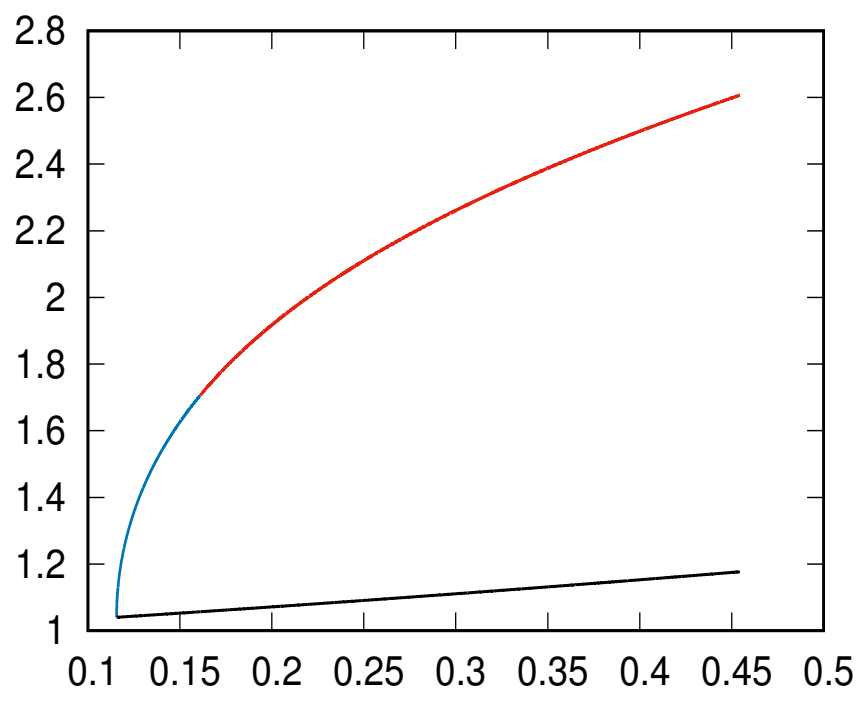

(a)

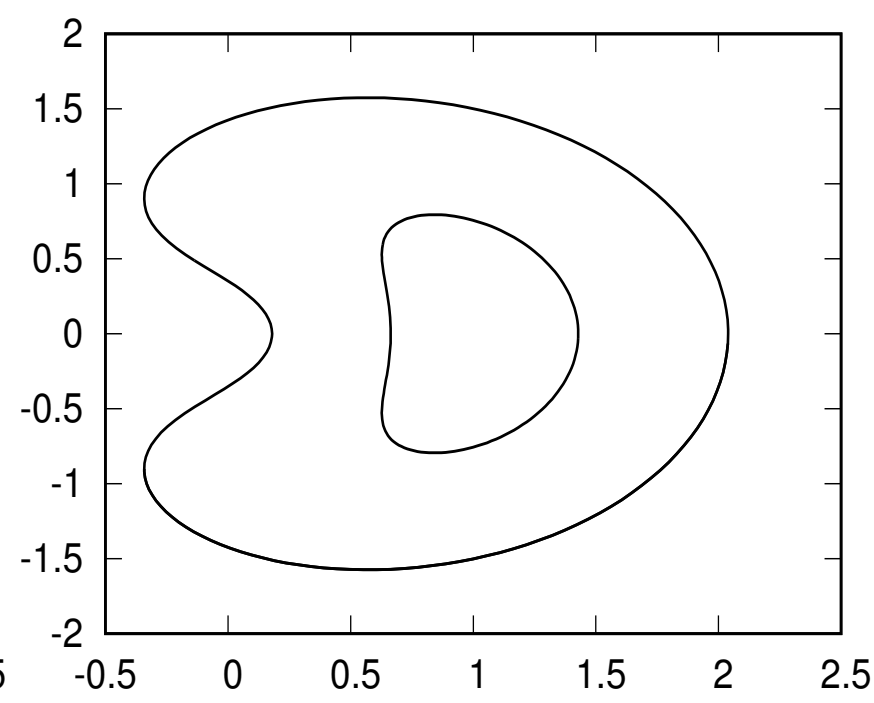

(b)

Figure 7: $h=h\left(L_{2}\right)$. (a) Two curves on the $(K, x)$ plane: for each $K$, the (lower) $x$ value of the equilibrium point $L_{2}$ and the (bigger) $x$ value of the initial condition of the bifurcated periodic orbit. The blue (red) points correspond to stable (unstable) periodic orbits. (b) Plane ( $x, y$ ). Bifurcated periodic orbits for $K=0.13$ and $K=0.23$

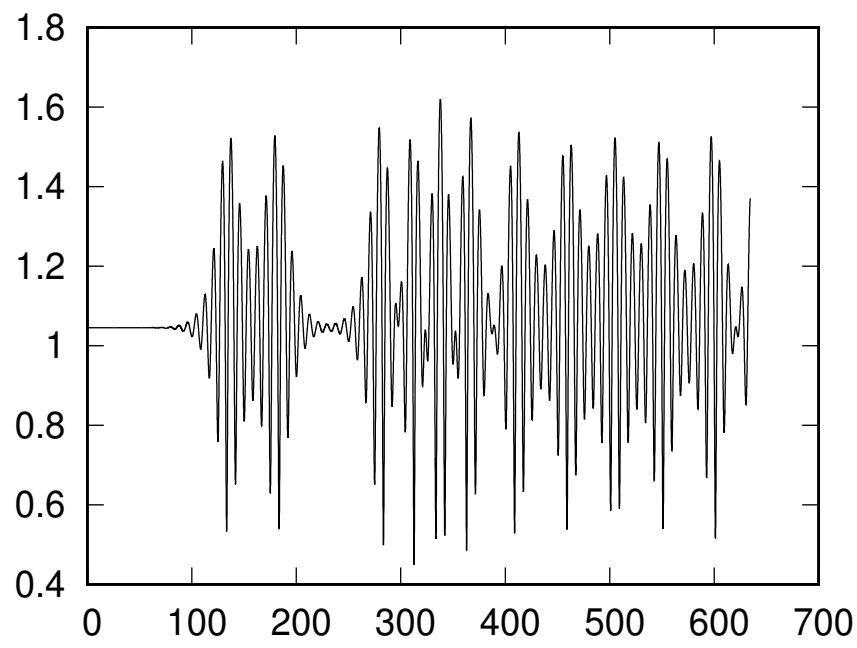

(a)

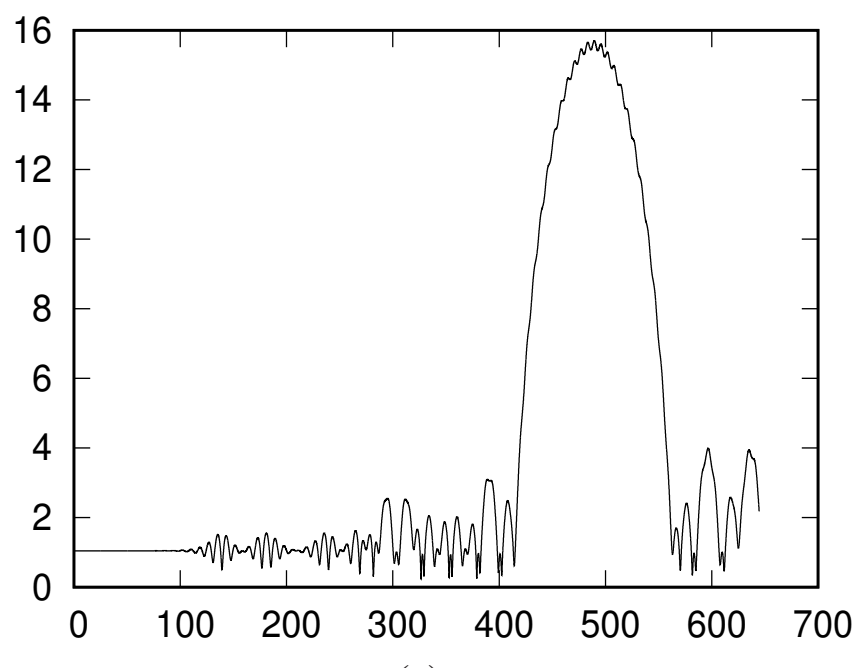

(c)

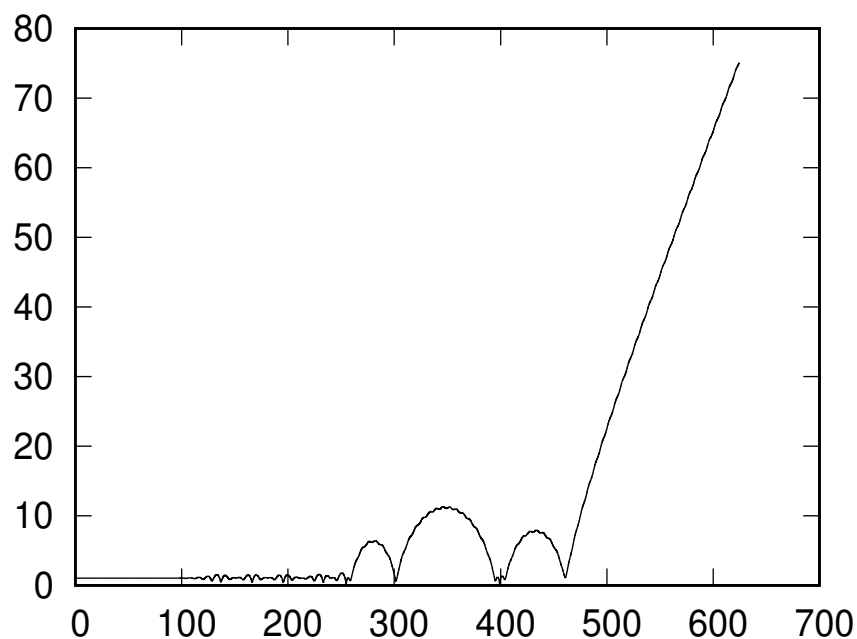

(b)

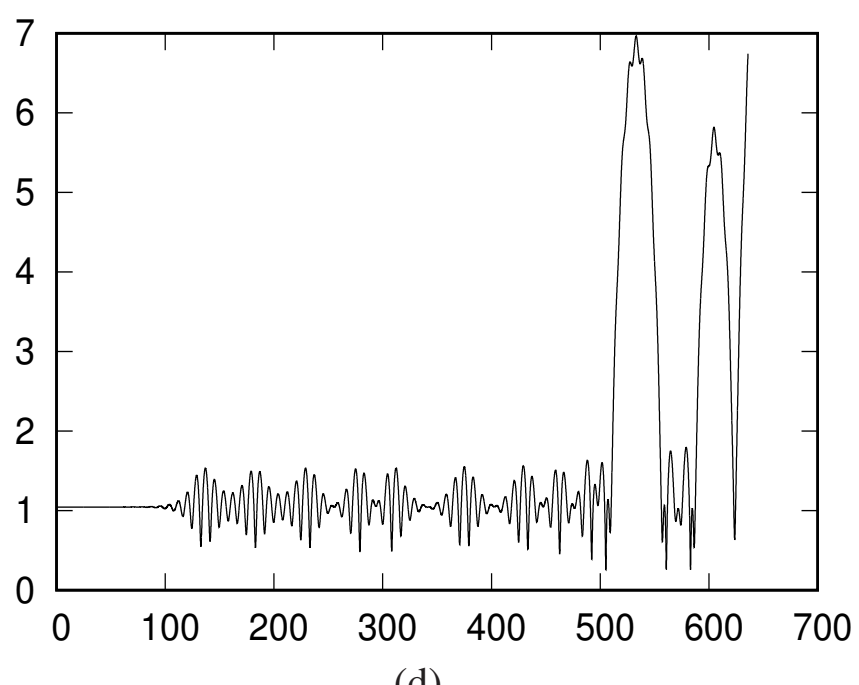

(d)

Figure 8: $K=0.13$. $(t, r)$ plots of some orbits in $W^{u}\left(L_{2}\right)$. (a) Confined orbit. (b) Slow ionizing orbit. Bottom: two different erratic orbits with several passages, (c) close to and (d) far from $L_{2}$. 


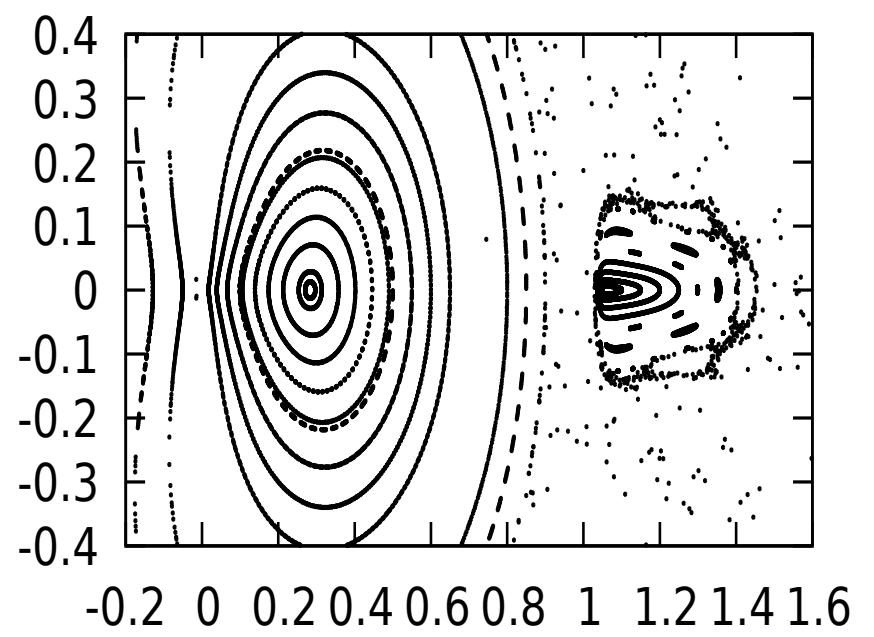

(a)

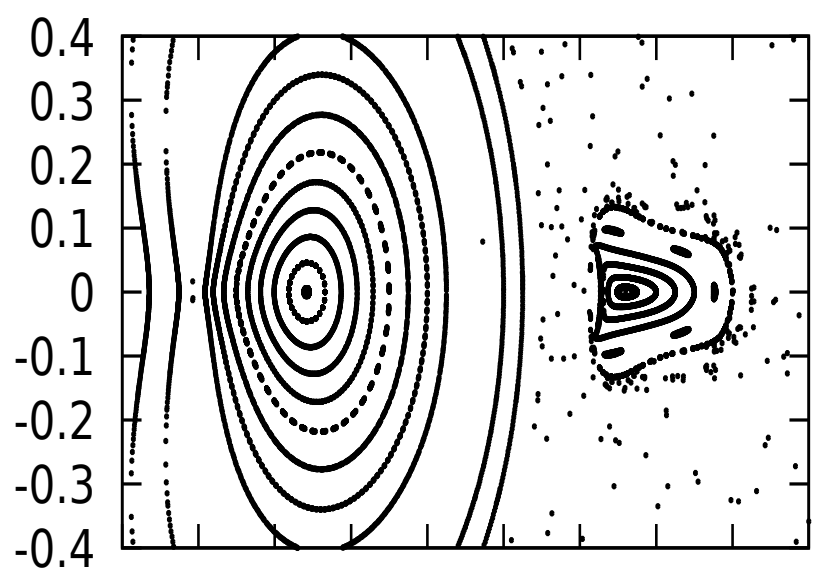

$\begin{array}{llllllll}-0.2 & 0 & 0.2 & 0.40 .60 .8 & 1 & 1.21 .4 & 1.6\end{array}$

(b)

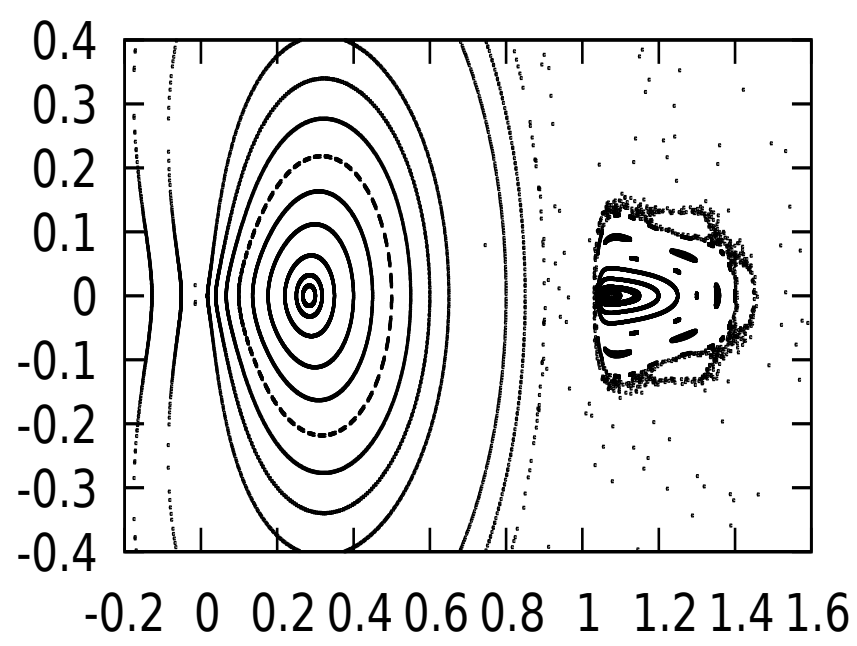

(c)

Figure 9: $K=0.115<K_{\text {crit }}$. PSP: the big stable region close to the origin is clearly seen. Notice: (a) the stable equilibrium point for $h=h\left(L_{2}\right)$, (b) the Liapunov stable periodic orbit for $h<h\left(L_{2}\right)$, and (c) the one for $h>h\left(L_{2}\right)$.

For a set of $m$ vectors $\eta_{1}, \eta_{2}, \ldots, \eta_{m} \in \mathbb{F}^{n}$

$$
M=\left(\eta_{1}\left|\eta_{2}\right| \cdots \mid \eta_{m}\right) \in \mathbb{M}_{n, m}(\mathbb{F})
$$

is the matrix having these vectors as columns.

As usual, the upper bar will denote complex conjugation, i.e., if $z \in \mathbb{C}$, then $\bar{z}=\operatorname{Re} z-\mathrm{i} \operatorname{Im} z$, whereas for matrices and vectors this convention extends componentwise, so if $A=\left(a_{i, j}\right) \in \mathbb{M}_{n, m}(\mathbb{C})$, then $\bar{A}=\left(\bar{a}_{i, j}\right) \in \mathbb{M}_{n, m}(\mathbb{C}), i=$ $1, \ldots, n, j=1, \ldots, m$; and we say that $\bar{A}$ is the complex conjugate (or simply, the conjugate) of $A$.

Let $\mathcal{S}: \mathbb{C}[[\nu, \alpha, \beta]] \rightarrow \mathbb{C}[[\nu, \alpha, \beta]]$ be the linear operator defined by

$$
F=\sum_{\ell, r, s} f_{\ell r_{1} r_{2} s_{1} s_{2}} \nu^{\ell} \alpha_{1}^{r_{1}} \alpha_{2}^{r_{2}} \beta_{1}^{s_{1}} \beta_{2}^{s_{2}} \mapsto \mathcal{S}(F):=\sum_{\ell, r, s}(-1)^{r_{1}+s_{2}} \bar{f}_{\ell s_{2} s_{1} r_{2} r_{1}} \nu^{\ell} \alpha_{1}^{r_{1}} \alpha_{2}^{r_{2}} \beta_{1}^{s_{1}} \beta_{2}^{s_{2}}
$$

We shall use the symbols $\mathbb{C}^{\mathcal{S}}[[\nu, \alpha, \beta]], \mathbb{E}_{\sigma}^{\mathcal{S}}[\nu, \alpha, \beta]$, and $\mathbb{E}_{\ell M N}^{\mathcal{S}}[\nu, \alpha, \beta]$ to refer the subspaces of $\mathbb{C}[[\nu, \alpha, \beta]], \mathbb{E}_{\sigma}[\nu, \alpha, \beta]$, and $\mathbb{E}_{\ell M N}[\nu, \alpha, \beta]$ respectively, that are invariant under $\mathcal{S}$, i. e., $F \in \mathbb{C}^{\mathcal{S}}[[\nu, \alpha, \beta]]\left(F \in \mathbb{E}_{\sigma}^{\mathcal{S}}[\nu, \alpha, \beta], F \in \mathbb{E}_{\ell M N}^{\mathcal{S}}[\nu, \alpha, \beta]\right)$ if and only if $F \in \mathbb{C}[[\nu, \alpha, \beta]]\left(F \in \mathbb{E}_{\sigma}[\nu, \alpha, \beta], F \in \mathbb{E}_{\ell M N}[\nu, \alpha, \beta]\right)$ and $\mathcal{S}(F)=F$. Then it is said that $F$ satisfies the $\mathcal{S}$-invariance or the $\mathcal{S}$-symmetries.

\section{Appendix AppendixB. Expansion of the Hamiltonian}

The goal of this appendix is to give the explicit expression of terms $\mathcal{H}_{j}(\mu, x, y), j=3,4, \ldots$ that appear in the expansion (19). Following [21], [47], [48]), first we expand the term $1 / r$ in Hamiltonian (5). Applying the change (17), we substitute $x$ by 
$\delta\left(1+x_{1}\right)$ and $y$ by $\delta x_{2}$ in $1 / r$. This yields

$$
\frac{1}{r}=\frac{1}{\sqrt{x^{2}+y^{2}}}=\frac{1 / \delta}{\sqrt{1+2 \frac{x-\delta}{\delta}+\left(\frac{x-\delta}{\delta}\right)^{2}+\left(\frac{y}{\delta}\right)^{2}}}=\frac{1 / \delta}{\sqrt{1-2\left(-\frac{x_{1}}{\rho}\right) \rho+\rho^{2}}}=\frac{1}{\delta} \sum_{\sigma=0}^{\infty} \rho^{\sigma} P_{\sigma}\left(-x_{1} / \rho\right),
$$

where $\rho^{2}:=x_{1}^{2}+x_{2}^{2}$ and $P_{\sigma}(t), \sigma=0,1,2, \ldots$ are the Legendre polynomials as generated by the recurrence relation

$$
\begin{aligned}
P_{0}(t) & =1, \quad P_{1}(t)=t, \\
P_{\sigma+1}(t) & =2 t P_{\sigma}(t)-P_{\sigma-1}(t)-\frac{1}{\sigma+1}\left(t P_{\sigma}(t)-P_{\sigma-1}(t)\right),
\end{aligned}
$$

with $\sigma=0,1,2, \ldots$ (see, for example [1], Chap. 12). If we define

$$
R_{\sigma}\left(x_{1}, x_{2}\right):=\rho^{\sigma} P_{\sigma}\left(-x_{1} / \rho\right)
$$

then, clearly,

$$
\frac{1}{r}=\frac{1}{\delta} \sum_{\sigma=3}^{\infty} R_{\sigma}\left(x_{1}, x_{2}\right)
$$

Furthermore, the recurrence relation,

$$
\begin{aligned}
R_{0}\left(x_{1}, x_{2}\right)= & 1, \quad R_{1}\left(x_{1}, x_{2}\right)=-x_{1}, \\
R_{\sigma+1}\left(x_{1}, x_{2}\right)= & -2 x_{1} R_{\sigma}\left(x_{1}, x_{2}\right)-\rho^{2} R_{\sigma-1}\left(x_{1}, x_{2}\right) \\
& +\frac{1}{\sigma+1}\left(x_{1} R_{\sigma}\left(x_{1}, x_{2}\right)+\rho^{2} R_{\sigma-1}\left(x_{1}, x_{2}\right)\right), \sigma=1,2, \ldots
\end{aligned}
$$

follows straightforward from (B.1). For example, for $\sigma=1,2,3$,

$$
\begin{array}{ll}
R_{2}\left(x_{1}, x_{2}\right)=x_{1}^{2}-\frac{x_{2}^{2}}{2}, & R_{3}\left(x_{1}, x_{2}\right)=-x_{1}^{3}+\frac{3}{2} x_{1} x_{2}^{2}, \\
R_{4}\left(x_{1}, x_{2}\right)=x_{1}^{4}-3 x_{1}^{2} x_{2}^{2}+\frac{3}{8} x_{2}^{4}, & R_{5}\left(x_{1}, x_{2}\right)=-x_{1}^{5}+5 x_{1}^{3} x_{2}^{2}-\frac{15}{8} x_{1} x_{2}^{4}, \ldots
\end{array}
$$

and induction shows that, for every $\sigma=0,1,2, \ldots, R_{\sigma}$ is a homogeneous polynomial of degree $\sigma$ in $x_{1}$ and $x_{2}$.

Now, if one substitutes the change (17) in the starting Hamiltonian (5), and replace $1 / r$ by the sum (B.2) obtains

$$
\begin{aligned}
H\left(\delta\left(1+x_{1}\right), \delta x_{2}, \frac{y_{1}}{\delta}, \delta+\frac{y_{2}}{\delta}\right)= & -\frac{\delta^{2}}{2}-\frac{1}{\delta}+K \delta-\frac{1}{\delta}\left(\delta^{3}-K \delta^{2}-1\right) x_{1}+\frac{1}{2 \delta^{2}}\left(y_{1}^{2}+y_{2}^{2}\right) \\
& +y_{1} x_{2}-y_{2} x_{1}-\frac{1}{\delta}\left(x_{1}^{2}-\frac{x_{2}^{2}}{2}\right)-\frac{1}{\delta} \sum_{\sigma=3}^{\infty} R_{\sigma}\left(x_{1}, x_{2}\right) .
\end{aligned}
$$

At this point, we recall that $\delta$ is a positive solution of Eq. (14) for $x>0$, so

$$
\delta^{3}-K \delta^{2}-1=0 .
$$

Therefore,

$$
\begin{aligned}
H\left(\delta\left(1+x_{1}\right), \delta x_{2}, \frac{y_{1}}{\delta}, \delta+\frac{y_{2}}{\delta}\right)= & H(\delta, 0,0, \delta)+\frac{1}{2 \delta^{2}}\left(y_{1}^{2}+y_{2}^{2}\right) \\
& +y_{1} x_{2}-y_{2} x_{1}-\frac{1}{\delta}\left(x_{1}^{2}-\frac{x_{2}^{2}}{2}\right)-\frac{1}{\delta} \sum_{\sigma=3}^{\infty} R_{\sigma}\left(x_{1}, x_{2}\right)
\end{aligned}
$$

(recall the definition (18)). On its turn, $H(\delta, 0,0, \delta)$ can be expanded in powers of $\mu$, with coefficients given by functions of $\delta_{0}$. Indeed,

$$
\begin{aligned}
H(\delta, 0,0, \delta)=-\frac{\delta^{2}}{2}-\frac{1}{\delta}+K \delta=\frac{\delta^{2}}{2}-\frac{2}{\delta} & =\frac{\delta_{0}^{2} / 2}{\left(1-\delta_{0} \mu\right)^{2}}-\frac{2}{\delta_{0}}+2 \mu \\
& =H\left(\delta_{0}, 0,0, \delta_{0}\right)+\left(2+\delta_{0}^{3}\right) \mu+\sum_{\sigma=2}^{\infty} \frac{\sigma+1}{2} \delta_{0}^{\sigma+2} \mu^{\sigma},
\end{aligned}
$$

where we have used that

$$
K \delta=\delta^{2}-\frac{1}{\delta}, \quad \delta=\frac{\delta_{0}}{1-\delta_{0} \mu},
$$


(see Eqs. (B.4) and (16)). Replacing $H(\delta, 0,0, \delta)$ on the right hand side of (B.5) by the right hand side of (B.6), taking into account that $1 / \delta=\left(1-\delta_{0} \mu\right) / \delta_{0}$ (see Eq. (B.7) above), and identifying the terms of the same (adapted, see (10)) degree in the resulting expansion, it is seen that the terms $\mathcal{H}_{\sigma}$ in the sum (18) are,

$$
\begin{aligned}
& \mathcal{H}_{2}(\mu, x, y)=\left(2+\delta_{0}^{3}\right) \mu+\frac{1}{2 \delta_{0}^{2}}\left(y_{1}^{2}+y_{2}^{2}\right)+x_{2} y_{1}-x_{1} y_{2}-\frac{1}{\delta_{0}}\left(x_{1}^{2}-\frac{x_{2}^{2}}{2}\right), \\
& \mathcal{H}_{3}(\mu, x, y)=-\frac{1}{\delta_{0}} R_{3}(x), \\
& \mathcal{H}_{4}(\mu, x, y)=\frac{3}{2} \delta_{0}^{4} \mu^{2}+\mu\left(x_{1}^{2}-\frac{x_{2}^{2}}{2}-\frac{y_{1}^{2}}{\delta_{0}}-\frac{y_{2}^{2}}{\delta_{0}}\right)-\frac{1}{\delta_{0}} R_{4}(x), \\
& \mathcal{H}_{5}(\mu, x, y)=\mu R_{3}(x)-\frac{1}{\delta_{0}} R_{5}(x), \\
& \mathcal{H}_{6}(\mu, x, y)=2 \delta_{0}^{5} \mu^{3}+\frac{\mu^{2}}{2}\left(y_{1}^{2}+y_{2}^{2}\right)+\mu R_{4}(x)-\frac{1}{\delta_{0}} R_{6}(x)
\end{aligned}
$$

and, for $\sigma=7,8, \ldots$

$$
\mathcal{H}_{\sigma}(\mu, x, y)=\frac{1-(-1)^{\sigma+1}}{8}(\sigma+2) \delta_{0}^{2+\sigma / 2} \mu^{\sigma / 2}+\mu R_{\sigma-2}(x)-\frac{1}{\delta_{0}} R_{\sigma}(x)
$$

\section{Appendix AppendixC. Linear reduction of the Hamiltonian system}

In this appendix, Lemma 1 below gives the form of transformation $z=\mathcal{U}(\zeta)$ that casts the quadratic part $\mathcal{H}_{2}$ in $(19)$ to Williamson's normal form (see references in Section 3.3). Next, the change to complex coordinates (20) is applied and, actually, is with respect to the quadratic part, $\mathscr{H}_{2}$, of the complexified Hamiltonian that we carry out the normal form computations (in Subsection 3.4 and in Appendix AppendixD). Notation and definitions used in this appendix were introduced in Subsection 3.1 and in Appendix AppendixA.

Lemma 1. Let $A \in \operatorname{sp}(4, \mathbb{R})$ with

$$
\operatorname{Spec}(A)=\left\{\lambda_{ \pm}= \pm \mathrm{i} \varpi\right\}, \quad \operatorname{dim} \operatorname{ker}\left(A-\lambda_{ \pm} I_{4}\right)=1,
$$

$\varpi \in \mathbb{R}, \varpi>0 ; z_{+}, w_{+} \in \mathbb{C}^{4}$ be the geometric and the generalized eigenvectors respectively of $\lambda_{+}=\mathrm{i} \varpi$, i.e.,

$$
A z=\lambda_{+} z_{+}, \quad\left(A-\lambda_{+} I_{4}\right) w_{+}=\lambda_{+} z_{+} .
$$

Let $\Delta, \epsilon$ and $\alpha$ be the quantities defined by,

$$
\Delta:=z_{+}^{\top} J_{2} \bar{w}_{+}, \quad \epsilon:=\operatorname{sign}(\Delta), \quad \alpha:=\frac{\mathrm{i}}{2 \Delta} w_{+}^{\top} J_{2} \bar{w}_{+}
$$

and $u_{1}, u_{2}, v_{1}, v_{2} \in \mathbb{R}^{4}$ the vectors

$$
\begin{array}{ll}
u_{1}=\sqrt{\frac{2}{|\Delta|}} \operatorname{Re} z_{+}, & u_{2}=\sqrt{\frac{2}{|\Delta|}} \operatorname{Im} z_{+}, \\
v_{1}=\epsilon \sqrt{\frac{2}{|\Delta|}}\left(-\alpha \operatorname{Im} z_{+}+\operatorname{Re} w_{+}\right), & v_{2}=\epsilon \sqrt{\frac{2}{|\Delta|}}\left(\alpha \operatorname{Re} z_{+}+\operatorname{Im} w_{+}\right) .
\end{array}
$$

Then,

(i) $\Delta \in \mathbb{R}, \alpha \in \mathbb{R}$.

(ii) $u_{1}, u_{2}, v_{1}, v_{2}$ form a symplectic basis, so $S=\left(u_{1}\left|u_{2}\right| v_{1} \mid v_{2}\right) \in \operatorname{Sp}(4, \mathbb{R})$.

(iii) The linear symplectic change $z=\mathcal{U}(\zeta):=S \zeta ; \zeta=(\xi, \eta), z=(x, y)$ with $\xi, \eta, x, y \in \mathbb{R}^{2}$; transforms:

(iii.1) The linear Hamiltonian system

$$
\dot{z}=A z,
$$

to its normal form

$$
\dot{\zeta}=\Lambda \zeta
$$

with

$$
\Lambda=S^{-1} A S=\left(\begin{array}{c|c}
\Omega & \epsilon I_{2} \\
\hline 0 & \Omega
\end{array}\right) \in \operatorname{sp}(4, \mathbb{R}), \quad \Omega=\left(\begin{array}{rr}
0 & \omega \\
-\omega & 0
\end{array}\right) .
$$


(iii.2) The quadratic Hamiltonian,

$$
\mathcal{H}_{2}(x, y)=-\frac{1}{2} z^{\top} J_{2} A z
$$

to its normal form

$$
K_{2}(\xi, \eta)=-\frac{1}{2} \zeta^{\top} J_{2} \Lambda \zeta=\frac{\epsilon}{2}\left(\eta_{1}^{2}+\eta_{2}^{2}\right)+\varpi\left(\eta_{1} \xi_{2}-\eta_{2} \xi_{1}\right) .
$$

PROOF. The results of this Lemma correspond, for Hamiltonian matrices, to the results stated for the symplectic ones in Appendix D of [7]. All the statements of Lemma 1 can be checked out straightforward from the very basic definitions of symplectic and Hamiltonian matrices and their properties.

Complexification of the Hamiltonian. Let $\zeta=\mathcal{V}(w):=\mathcal{L} w ; \zeta=(\xi, \eta), w=(q, p)$, with $\xi=\left(\xi_{1}, \xi_{2}\right), \eta=\left(\eta_{1}, \eta_{2}\right)$, $q=\left(q_{1}, q_{2}\right), p=\left(p_{1}, p_{2}\right) \in \mathbb{C}^{2}$, and $\mathcal{L} \in \mathbb{M}_{4}(\mathbb{C})$; be the change to complex coordinates (20) written in matrix notation. Clearly,

$$
\mathcal{L}=\left(\begin{array}{cccc}
1 / \sqrt{2} & 0 & 0 & -1 / \sqrt{2} \\
\mathrm{i} / \sqrt{2} & 0 & 0 & \mathrm{i} / \sqrt{2} \\
0 & 1 / \sqrt{2} & 1 / \sqrt{2} & 0 \\
0 & \mathrm{i} / \sqrt{2} & -\mathrm{i} / \sqrt{2} & 0
\end{array}\right) \in \operatorname{Sp}(\mathbb{C}, 4),
$$

so, the composition of both changes is $z=\Xi(w):=\mathcal{U} \circ \mathcal{V}(w)=\mathcal{M} w$, with

$$
\mathcal{M}:=S \mathcal{L}=\left(\begin{array}{cccc}
-\mathrm{i} \tilde{A} \sqrt{10} & -\tilde{A} \sqrt{2} & -\tilde{A} \sqrt{2} & -\mathrm{i} \tilde{A} \sqrt{10} \\
5 \tilde{A} \sqrt{2} & \mathrm{i} \tilde{A} \sqrt{10} & -\mathrm{i} \tilde{A} \sqrt{10} & -5 \tilde{A} \sqrt{2} \\
-10 \tilde{B} \sqrt{2} & -7 \mathrm{i} \tilde{B} \sqrt{10} & 7 \mathrm{i} \tilde{B} \sqrt{10} & 10 \tilde{B} \sqrt{2} \\
2 \mathrm{i} \tilde{B} \sqrt{10} & 7 \tilde{B} \sqrt{2} & 7 \tilde{B} \sqrt{2} & 2 \mathrm{i} \tilde{B} \sqrt{10}
\end{array}\right) \in \mathrm{Sp}(\mathbb{C}, 4), \tilde{A}=\frac{3^{5 / 6}}{15}, \tilde{B}=\frac{3^{1 / 6}}{20} .
$$

Let $\mathscr{H}(\mu, w):=\mathcal{H}(\mu, \Xi(w))$ be the complexified Hamiltonian. Thus, (21) is the expansion of $\mathscr{H}$, with $\mathscr{H}_{\sigma} \in \mathbb{E}_{\sigma}[\mu, q, p]$. Particularly, one checks immediately that the quadratic term, $\mathscr{H}_{2}$ is given by (22).

Remark AppendixC.1. Here we stress that,

(i) $\eta_{1}^{2}+\eta_{2}^{2}=2 q_{2} p_{1}$ and $\eta_{1} \xi_{2}-\eta_{2} \xi_{1}=\mathrm{i}\left(q_{1} p_{1}+q_{2} p_{2}\right)$ and, when the change (C.2) is applied to the particular Hamiltonian (19) then, $\tilde{\alpha}=25 / 8, \varpi=\sqrt{5} / 3$, and $\epsilon=1$.

(ii) Substitution of (C.2) in the recurrence relations (B.3) gives the expansion (21). Thus, if $\mathcal{R}_{\sigma}:=R_{\sigma} \circ \Xi$; then

$$
\begin{gathered}
\mathcal{R}_{0}(q, p)=1, \\
\mathcal{R}_{1}(q, p)=\mathrm{i} \frac{3^{5 / 6}}{15} \sqrt{10} q_{1}+\frac{3^{5 / 6}}{15} \sqrt{2} q_{2}+\frac{3^{5 / 6}}{15} \sqrt{2} p_{1}+\mathrm{i} \frac{3^{5 / 6}}{15} \sqrt{10} p_{2}, \\
\mathcal{R}_{\sigma+1}(q, p)=2 \mathcal{R}_{1}(q, p) \mathcal{R}_{\sigma}(q, p)-\varrho^{2}(q, p) \mathcal{R}_{\sigma-1}(q, p)+\frac{1}{\sigma+1}\left(-\mathcal{R}_{1}(q, p) \mathcal{R}_{\sigma}(q, p)+\varrho^{2}(q, p) \mathcal{R}_{\sigma-1}(q, p)\right), \\
\sigma=1,2, \ldots ; \text { with } \varrho=\rho \circ \Xi \text {, that is } \\
\varrho(q, p)=\frac{8}{15} 3^{2 / 3} q_{1}^{2}+\frac{8}{25} \mathrm{i} 3^{2 / 3} \sqrt{5} p_{1} p_{2}-\frac{16}{75} \mathrm{i} 3^{2 / 3} \sqrt{5} q_{2} p_{2}-\frac{8}{5} 3^{2 / 3} q_{1} p_{2}-\frac{16}{75} \mathrm{i} 3^{2 / 3} \sqrt{5} q_{1} p_{1} \\
\quad-\frac{8}{75} 3^{2 / 3} q_{2}^{2}+\frac{8}{25} 3^{2 / 3} q_{2} p_{1}+\frac{8}{25} \mathrm{i} 3^{2 / 3} \sqrt{5} q_{1} q_{2}-\frac{8}{75} 3^{2 / 3} p_{1}^{2}+\frac{8}{15} 3^{2 / 3} p_{2}^{2} .
\end{gathered}
$$

(iii) If the power series $K(\mu, z) \in \mathbb{C}[[\mu, x, y]]$ has real coefficients, its complexification $F(\mu, w)=K(\mu, \Xi(w))$ satisfies the reality condition, i.e., $F(\mu, w) \in \mathbb{C}^{\mathcal{S}}[[\mu, q, p]]$. Conversely, if $F \in \mathbb{C}^{\mathcal{S}}[[\mu, q, p]]$, then $F\left(\mu, \Xi^{-1}(z)\right) \in \mathbb{C}[[\mu, x, y]]$ is a power series with real coefficients. Furthermore, it is worth noting that the Poisson bracket preserves the $\mathcal{S}$ invariance. More precisely, if $F, G \in \mathbb{C}^{\mathcal{S}}[[\mu, q, p]]$, then $\{F, G\} \in \mathbb{C}^{\mathcal{S}}[[\mu, q, p]]$ (see for example [21], [41], Remark 3.4 ; or [52]).

\section{Appendix AppendixD. Proof of Proposition 1}

Here we develop the proof of Proposition 1. In addition, some remarks are given at the end. The notation and definitions used throughout this appendix were introduced in subsection 3.1 and in Appendix AppendixA. Moreover, the conventions established at Remark 3 will apply along the proof.

So, let us consider a monomial $\alpha=\mu^{\ell} q^{m} p^{n}$, with $2 \ell+|m|_{1}+|n|_{1}=\sigma$, i.e., of adapted degree $\sigma \geq 3$. The action of the operator $L_{Z_{2}}$ on $\alpha$ gives,

$$
L_{Z_{2}} \alpha=\left(\Omega+m_{1} \frac{q_{2}}{q_{1}}-n_{2} \frac{p_{1}}{p_{2}}\right) \alpha,
$$

being $\Omega=\Omega_{|m|_{1}|n|_{1}}:=\mathrm{i} \omega\left(|m|_{1}-|n|_{1}\right)$, and where it assumed that the quotient $q_{2} / q_{1}$ is not present when $m_{1}=0$ (the same convention applies for the quotient $p_{1} / p_{2}$ when $n_{2}=0$ ). 
Remark AppendixD.1 (on the values of $\ell$ ). We note here that no linear terms in the coordinates $(q, p)$ can appear in any of the new transformed Hamiltonians, since for any $\mu$, the origin $(q, p)=(0,0)$ is an equilibrium point and, in the reduction process to normal form all the changes that we made are close to the identity. Therefore, $|m|_{1}+|n|_{1}$ cannot be one at any of the successive transformed Hamiltonians along the reduction process, so the equilibrium point remains at the origin at each step. Thus, at the homogeneous polynomials

$$
\mathscr{H}_{\kappa}^{(\sigma-2)}=\sum_{2 \ell+|m|_{1}+|n|_{1}=\kappa} \mathscr{H}_{\ell m n}^{(\sigma-2)} \mu^{\ell} q^{m} p^{n}
$$

of (adapted) degree $\kappa=\sigma, \sigma+1, \ldots, s$ (see (D.31)), the index $\ell$ can vary only in the range $\ell=0, \ldots, 3\lfloor\kappa / 2\rfloor-\kappa$ $(\sigma=3,4, \ldots)$, where $\lfloor\cdot\rfloor$ stands for the integer part.

If follows from (D.1) that $L_{Z_{2}}$ leaves each subspace $\mathbb{E}_{\ell M N} \subseteq \mathbb{E}_{\sigma}$ invariant. Therefore, we can fix $\ell, M, N \in \mathbb{N}_{0}$ and focus on solving the projections of equations (25) onto the subspace $\mathbb{E}_{\ell M N}$; we mean, given $F \in \mathbb{E}_{\ell M N}$, we look for $G, Z \in \mathbb{E}_{\ell M N}$ such that the homological equation

$$
L_{Z_{2}} G+Z=F
$$

holds. It turns out that, when $\Omega \neq 0$ (i.e., when $M \neq N$ ) then $F \in$ Range $\left.L_{Z_{2}}\right|_{\mathbb{E}_{\ell M N}}$ and we can set $Z=0$ in equation above. Otherwise, when $\Omega=0$ (i.e., when $M=N$ ), then resonant monomials appear generically. We analyze both cases below.

Remark AppendixD.2. Prior to continuing with our analysis, let us point out that, actually, when one writes the Eq. (D.2) explicitly, the term $\mu^{\ell}$ appears as a product at both sides, and can be simplified. Hence, abusing notation, this factor has been omitted in the expansions of the polynomials in $\mathbb{E}_{\ell M N}$.

The case $\Omega \neq 0(M \neq N)$. Let $U \in \mathbb{E}_{\ell M N}$, with $\ell, M, N$ fixed, then

$$
U=\sum_{\substack{|m|_{1}=M \\|n|_{1}=N}} u_{\ell m_{1} m_{2} n_{1} n_{2}} q_{1}^{m_{1}} q_{2}^{m_{2}} p_{1}^{n_{1}} p_{2}^{n_{2}}=\sum_{\substack{0 \leq m \leq M \\ 0 \leq n \leq N}} u_{\ell, m, M-m, N-n, n} q_{1}^{m} q_{2}^{M-m} p_{1}^{N-n} p_{2}^{n},
$$

(the factor $\mu^{\ell}$ is omitted, as Remark AppendixD.2 warns). Thus, we can set $u_{m n}:=u_{\ell, m, M-m, N-n, n}$ for $0 \leq m \leq M$, $0 \leq n \leq N$; so, for every $\ell$ fixed, any monomial in $U$ is specified by just a pair of indices. Now, let $u \in \mathbb{M}_{(M+1) \times(N+1), 1}(\mathbb{C})$ be the array holding the coefficients $u_{m n}$ ordered as follows: $u_{m n} \prec u_{m^{\prime} n^{\prime}}\left(u_{m n}\right.$ precedes $\left.u_{m^{\prime} n^{\prime}}\right)$ if $m>m^{\prime}$ or, when $m=m^{\prime}$, if $n>n^{\prime}$.

Example 1. For instance, if $M=3$ and $N=2$; then

$$
u_{3,2} \prec u_{3,1} \prec u_{3,0} \prec u_{2,2} \prec u_{2,1} \prec u_{2,0} \prec u_{1,2} \prec u_{1,1} \prec u_{1,0} \prec u_{0,2} \prec u_{0,1} \prec u_{0,0} .
$$

Yet we can define the block-array $u^{\top}=\left(u_{M} u_{M-1} \cdots u_{0}\right) \in \mathbb{C}^{N+1} \times \mathbb{C}^{N+1} \times \cdots \times \mathbb{C}^{N+1}$, with

$$
u_{j}^{\top}=\left(u_{j, N}, u_{j, N-1}, \ldots, u_{j, 0}\right) \in \mathbb{C}^{N+1}
$$

for $j=M, M-1, \ldots, 0$. Therefore, to find the solution of (D.2) we set $Z_{\ell M N}=0$ and solve a linear system $A g=f$; with $A \in \mathbb{M}_{(M+1) \times(N+1)}(\mathbb{C}) ; f, g \in \mathbb{C}^{(M+1) \times(N+1)}$; which, using the notation just described, can be written blockwise and looks

$$
\left(\begin{array}{cccccc}
D_{N} & & & & & \\
E_{M} & D_{N} & & & & \\
& E_{M-1} & D_{N} & & & \\
& \ddots & & \ddots & & \\
& & \ddots & & \ddots & \\
& & & E_{2} & & D_{N} \\
& & & & E_{1} & D_{N}
\end{array}\right)\left(\begin{array}{c}
g_{M} \\
g_{M-1} \\
g_{M-2} \\
\vdots \\
\vdots \\
g_{1} \\
g_{0}
\end{array}\right)=\left(\begin{array}{c}
f_{M} \\
f_{M-1} \\
f_{M-2} \\
\vdots \\
\vdots \\
f_{1} \\
f_{0}
\end{array}\right),
$$

where $E_{j}=j I_{N+1} \in \mathbb{M}_{N+1}(\mathbb{C}), i, j=M, M-1, \ldots, 1 ; D_{N}=\Omega I_{N+1}-P_{N} \in \mathbb{M}_{N+1}(\mathbb{C})$ with $\left(P_{N}\right)_{i, j}=N-i+2$ if $i=j+1$ for $j=1, \ldots, N$, or $\left(P_{N}\right)_{i, j}=0$ otherwise. From the band structure of $A$, we see that it is a lower triangular matrix with all the elements on its diagonal equal to $\Omega \neq 0$, so the linear system (D.3) has exactly one solution.

Remark AppendixD.3 (Reality conditions). It is straightforward to show that $\bar{g}_{\ell, m, M-m, N-n, n}=(-1)^{m+n} g_{\ell, n, N-n, M-m, m}$, for $0 \leq m \leq M, 0 \leq n \leq N, 2 \ell+M+N=\sigma$. Hence $G_{\sigma}^{+} \in \mathbb{E}_{\sigma}^{\mathcal{S}}$.

Example 2. If, as in Example 1, we take $M=3, N=2$; then $\Omega=\mathrm{i} \omega(3-2)=\mathrm{i} \omega$ and

$$
P_{2}=\left(\begin{array}{ccc}
0 & 0 & 0 \\
2 & 0 & 0 \\
0 & 1 & 0
\end{array}\right), \quad D_{2}=\left(\begin{array}{ccc}
\mathrm{i} \omega & & \\
-2 & \mathrm{i} \omega & \\
& -1 & \mathrm{i} \omega
\end{array}\right), \quad E_{j}=\left(\begin{array}{ccc}
j & & \\
& j & \\
& j
\end{array}\right), j=1,2,3 ; \quad A=\left(\begin{array}{ccc}
D_{2} & & \\
E_{3} & D_{2} & \\
& E_{2} & D_{2} \\
& E_{1} & D_{2}
\end{array}\right)
$$


Therefore, for this case in point, the system (D.3) is written as

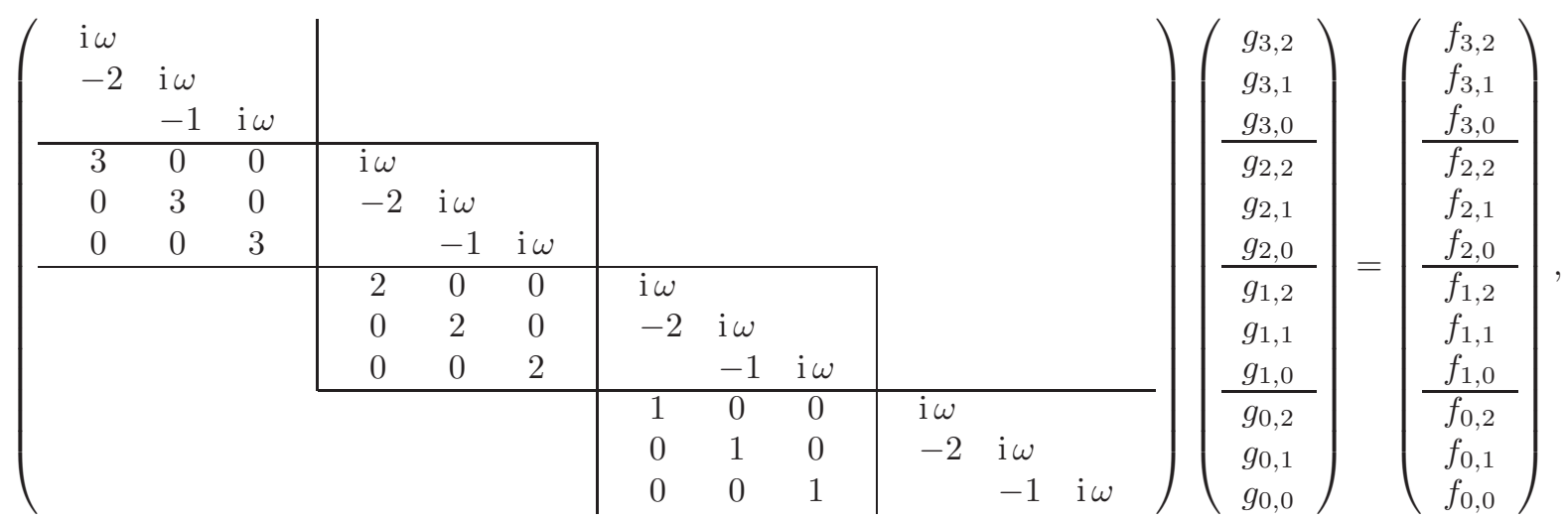

and it is seen at once -as we stated before for the general case- that $\operatorname{det} A \neq 0$, so the coefficients of the generating function are uniquely determined.

Hence, we can write the component $G_{\sigma}^{+} \in \mathbb{E}_{\sigma}^{+}$of the generating function $G_{\sigma}$ in the form,

$$
G_{\sigma}^{+}(\mu, q, p)=\sum_{\substack{2 \ell+M+N=\sigma \\ M \neq N}} G_{\ell M N}=\sum_{\substack{2 \ell+M+N=\sigma \\ M \neq N}}\left(\sum_{\substack{0 \leq m \leq M \\ 0 \leq n \leq N}} g_{\ell, m, M-m, N-n, n} q_{1}^{m} q_{2}^{M-m} p_{1}^{N-n} p_{2}^{n}\right) \mu^{\ell}
$$

This completes the proof of the part (a) of Proposition 1.

Example 3 (Computation of $G_{3}, \mathscr{H}_{4}^{(1)}$ and $G_{4}^{+}$). For $s=3$, the right hand side of (23) is given by

$$
\begin{aligned}
\mathscr{H}_{3}^{(0)}(\mu, q, p)= & \mathrm{i} \frac{34}{225} 3^{5 / 6} \sqrt{10} q_{1}^{3}-\frac{2}{25} 3^{5 / 6} \sqrt{2} q_{1}^{2} q_{2}+\mathrm{i} \frac{2}{125} 3^{5 / 6} \sqrt{10} q_{1} q_{2}^{2}-\frac{34}{1125} 3^{5 / 6} \sqrt{2} q_{2}^{3}-\mathrm{i} \frac{2}{25} 3^{5 / 6} \sqrt{10} q_{1}^{2} p_{2} \\
& +\frac{34}{75} 3^{5 / 6} \sqrt{2} q_{1}^{2} p_{1}-\frac{4}{25} 3^{5 / 6} \sqrt{2} q_{1} q_{2} p_{2}+\mathrm{i} \frac{4}{125} 3^{5 / 6} \sqrt{10} q_{1} q_{2} p_{1}-\mathrm{i} \frac{34}{375} 3^{5 / 6} \sqrt{10} q_{2}^{2} p_{2}+\frac{2}{125} 3^{5 / 6} \sqrt{2} q_{2}^{2} p_{1} \\
& -\mathrm{i} \frac{2}{25} 3^{5 / 6} \sqrt{10} q_{1} p_{2}^{2}-\frac{4}{25} 3^{5 / 6} \sqrt{2} q_{1} p_{1} p_{2}-\mathrm{i} \frac{34}{375} 3^{5 / 6} \sqrt{10} q_{1} p_{1}^{2}+\frac{34}{75} 3^{5 / 6} \sqrt{2} q_{2} p_{2}^{2}+\mathrm{i} \frac{4}{125} 3^{5 / 6} \sqrt{10} q_{2} p_{1} p_{2} \\
& +\frac{2}{125} 3^{5 / 6} \sqrt{2} q_{2} p_{1}^{2}+\mathrm{i} \frac{34}{225} 3^{5 / 6} \sqrt{10} p_{2}^{3}-\frac{2}{25} 3^{5 / 6} \sqrt{2} p_{1} p_{2}^{2}+\mathrm{i} \frac{2}{125} 3^{5 / 6} \sqrt{10} p_{1}^{2} p_{2}-\frac{34}{1125} 3^{5 / 6} \sqrt{2} p_{1}^{3} .
\end{aligned}
$$

Then, setting up the homological equations (D.2) -for $\ell=0$, taking $M=3, N=0 ; M=2, N=1 ; M=1, N=2$; $M=0, N=3-$, and solving the corresponding linear systems of type (D.3) one gets $Z_{3}=0$ and $G_{3}=G_{3}^{+}+G_{3}^{0}$, with

$$
\begin{aligned}
G_{3}^{+}(\mu, q, p)= & \frac{34}{225} 3^{5 / 6} \sqrt{2} q_{1}^{3}+\mathrm{i} \frac{8}{75} 3^{5 / 6} \sqrt{10} q_{1}^{2} q_{2}-\frac{74}{375} 3^{5 / 6} \sqrt{2} q_{1} q_{2}^{2}-\mathrm{i} \frac{188}{5625} 3^{5 / 6} \sqrt{10} q_{2}^{3} \\
& -\frac{6}{25} 3^{5 / 6} \sqrt{2} q_{1}^{2} p_{2}-\mathrm{i} \frac{16}{125} 3^{5 / 6} \sqrt{10} q_{1}^{2} p_{1}-\mathrm{i} \frac{24}{125} 3^{5 / 6} \sqrt{10} q_{1} q_{2} p_{2}+\frac{36}{125} 3^{5 / 6} \sqrt{2} q_{1} q_{2} p_{1} \\
& +\frac{38}{125} 3^{5 / 6} \sqrt{2} q_{2}^{2} p_{2}-\mathrm{i} \frac{12}{625} 3^{5 / 6} \sqrt{10} q_{2}^{2} p_{1}+\frac{6}{25} 3^{5 / 6} \sqrt{2} q_{1} p_{2}^{2}+\mathrm{i} \frac{24}{125} 3^{5 / 6} \sqrt{10} q_{1} p_{1} p_{2} \\
& -\frac{38}{125} 3^{5 / 6} \sqrt{2} q_{1} p_{1}^{2}+\mathrm{i} \frac{16}{125} 3^{5 / 6} \sqrt{10} q_{2} p_{2}^{2}-\frac{36}{125} 3^{5 / 6} \sqrt{2} q_{2} p_{1} p_{2}+\mathrm{i} \frac{12}{625} 3^{5 / 6} \sqrt{10} q_{2} p_{1}^{2} \\
& -\frac{34}{225} 3^{5 / 6} \sqrt{2} p_{2}^{3}-\mathrm{i} \frac{8}{75} 3^{5 / 6} \sqrt{10} p_{1} p_{2}^{2}+\frac{74}{375} 3^{5 / 6} \sqrt{2} p_{1}^{2} p_{2}+\mathrm{i} \frac{188}{5625} 3^{5 / 6} \sqrt{10} p_{1}^{3},
\end{aligned}
$$


and $G_{3}^{0}=0$. Furthermore, as

$$
\begin{aligned}
\mathscr{H}_{4}^{(0)}(\mu, q, p)= & \frac{27}{32} 3^{2 / 3} \mu^{2}-\frac{11}{15} 3^{2 / 3} \mu q_{1}^{2}-\mathrm{i} \frac{16}{25} 3^{2 / 3} \sqrt{5} \mu q_{1} q_{2}+\frac{56}{75} 3^{2 / 3} \mu q_{2}^{2}+\frac{6}{5} 3^{2 / 3} \mu q_{1} p_{2}+\mathrm{i} \frac{14}{25} 3^{2 / 3} \sqrt{5} \mu q_{1} p_{1} \\
& +\mathrm{i} \frac{14}{25} 3^{2 / 3} \sqrt{5} \mu q_{2} p_{2}-\frac{51}{25} 3^{2 / 3} \mu q_{2} p_{1}-\frac{11}{15} 3^{2 / 3} \mu p_{2}^{2}-\mathrm{i} \frac{16}{25} 3^{2 / 3} \sqrt{5} \mu p_{1} p_{2}+\frac{56}{75} 3^{2 / 3} \mu p_{1}^{2} \\
& -\frac{203}{225} 3^{2 / 3} q_{1}^{4}-\mathrm{i} \frac{268}{1125} 3^{2 / 3} \sqrt{5} q_{1}^{3} q_{2}+\frac{86}{375} 3^{2 / 3} q_{1}^{2} q_{2}^{2}+\mathrm{i} \frac{268}{5625} 3^{2 / 3} \sqrt{5} q_{1} q_{2}^{3}-\frac{203}{5625} 3^{2 / 3} q_{2}^{4} \\
& +\frac{268}{225} 3^{2 / 3} q_{1}^{3} p_{2}+\mathrm{i} \frac{812}{1125} 3^{2 / 3} \sqrt{5} q_{1}^{3} p_{1}+\mathrm{i} \frac{172}{375} 3^{2 / 3} \sqrt{5} q_{1}^{2} q_{2} p_{2}-\frac{268}{375} 3^{2 / 3} q_{1}^{2} q_{2} p_{1}-\frac{268}{375} 3^{2 / 3} q_{1} q_{2}^{2} p_{2} \\
& -\mathrm{i} \frac{172}{1875} 3^{2 / 3} \sqrt{5} q_{1} q_{2}^{2} p_{1}-\mathrm{i} \frac{812}{5625} 3^{2 / 3} \sqrt{5} q_{2}^{3} p_{2}+\frac{268}{5625} 3^{2 / 3} q_{2}^{3} p_{1}-\frac{86}{75} 3^{2 / 3} q_{1}^{2} p_{2}^{2}-\mathrm{i} \frac{268}{375} 3^{2 / 3} \sqrt{5} q_{1}^{2} p_{1} p_{2} \\
& +\frac{406}{375} 3^{2 / 3} q_{1}^{2} p_{1}^{2}-\mathrm{i} \frac{268}{375} 3^{2 / 3} \sqrt{5} q_{1} q_{2} p_{2}^{2}+\frac{344}{375} 3^{2 / 3} q_{1} q_{2} p_{1} p_{2}+\mathrm{i} \frac{268}{1875} 3^{2 / 3} \sqrt{5} q_{1} q_{2} p_{1}^{2}+\frac{406}{375} 3^{2 / 3} q_{2}^{2} p_{2}^{2} \\
& +\mathrm{i} \frac{268}{1875} 3^{2 / 3} \sqrt{5} q_{2}^{2} p_{1} p_{2}-\frac{86}{1875} 3^{2 / 3} q_{2}^{2} p_{1}^{2}+\frac{268}{225} 3^{2 / 3} q_{1} p_{2}^{3}+\mathrm{i} \frac{172}{375} 3^{2 / 3} \sqrt{5} q_{1} p_{1} p_{2}^{2}-\frac{268}{375} 3^{2 / 3} q_{1} p_{1}^{2} p_{2} \\
& -\mathrm{i} \frac{812}{5625} 3^{2 / 3} \sqrt{5} q_{1} p_{1}^{3}+\mathrm{i} \frac{812}{1125} 3^{2 / 3} \sqrt{5} q_{2} p_{2}^{3}-\frac{268}{375} 3^{2 / 3} q_{2} p_{1} p_{2}^{2}-\mathrm{i} \frac{172}{1875} 3^{2 / 3} \sqrt{5} q_{2} p_{1}^{2} p_{2}+\frac{268}{5625} 3^{2 / 3} q_{2} p_{1}^{3} \\
& -\frac{203}{225} 3^{2 / 3} p_{2}^{4}-\mathrm{i} \frac{268}{1125} 3^{2 / 3} \sqrt{5} p_{1} p_{2}^{3}+\frac{86}{375} 3^{2 / 3} p_{1}^{2} p_{2}^{2}+\mathrm{i} \frac{268}{5625} 3^{2 / 3} \sqrt{5} p_{1}^{3} p_{2}-\frac{203}{5625} 3^{2 / 3} p_{1}^{4},
\end{aligned}
$$

application of Algorithm AppendixD.1 yields,

$$
\begin{aligned}
\mathscr{H}_{4}^{(1)} & =\mathscr{H}_{4}^{(0)}+\frac{1}{1 !}\left\{\mathscr{H}_{3}^{(0)}, G_{3}\right\}+\frac{1}{2 !}\left\{\left\{Z_{2}, G_{3}\right\}, G_{3}\right\} \\
& =\mathscr{H}_{2,0,0}^{(1)}+\mathscr{H}_{1,2,0}^{(1)}+\mathscr{H}_{1,1,1}^{(1)}+\mathscr{H}_{1,0,2}^{(1)}+\mathscr{H}_{0,4,0}^{(1)}+\mathscr{H}_{0,3,1}^{(1)}+\mathscr{H}_{0,2,2}^{(1)}+\mathscr{H}_{0,1,3}^{(1)}+\mathscr{H}_{0,0,4}^{(1)}
\end{aligned}
$$

with $\mathscr{H}_{\ell M N}^{(1)} \in \mathbb{E}_{\ell M N}$. Explicitly,

$$
\begin{aligned}
& \mathscr{H}_{2,0,0}^{(1)}(\mu, q, p)=\frac{27}{32} 3^{2 / 3} \mu^{2}, \\
& \mathscr{H}_{1,2,0}^{(1)}(\mu, q, p)=-\frac{11}{15} 3^{2 / 3} \mu q_{1}^{2}-\mathrm{i} \frac{16}{25} 3^{2 / 3} \sqrt{5} \mu q_{1} q_{2}+\frac{56}{75} 3^{2 / 3} \mu q_{2}^{2}, \\
& \mathscr{H}_{1,1,1}^{(1)}(\mu, q, p)=\frac{6}{5} 3^{2 / 3} \mu q_{1} p_{2}+\mathrm{i} \frac{14}{25} 3^{2 / 3} \sqrt{5} \mu q_{1} p_{1}+\mathrm{i} \frac{14}{25} 3^{2 / 3} \sqrt{5} \mu q_{2} p_{2}-\frac{51}{25} 3^{2 / 3} \mu q_{2} p_{1}, \\
& \mathscr{H}_{1,0,2}^{(1)}(\mu, q, p)=-\frac{11}{15} 3^{2 / 3} \mu p_{2}^{2}-\mathrm{i} \frac{16}{25} 3^{2 / 3} \sqrt{5} \mu p_{1} p_{2}+\frac{56}{75} 3^{2 / 3} \mu p_{1}^{2} \text {, } \\
& \mathscr{H}_{0,4,0}^{(1)}(\mu, q, p)=-\frac{4043}{5625} 3^{2 / 3} q_{1}^{4}-\mathrm{i} \frac{3916}{28125} 3^{2 / 3} \sqrt{5} q_{1}^{3} q_{2}+\frac{1474}{3125} 3^{2 / 3} q_{1}^{2} q_{2}^{2}+\mathrm{i} \frac{748}{140625} 3^{2 / 3} \sqrt{5} q_{1} q_{2}^{3}+\frac{4453}{140625} 3^{2 / 3} q_{2}^{4}, \\
& \mathscr{H}_{0,3,1}^{(1)}(\mu, q, p)=\frac{476}{1125} 3^{2 / 3} q_{1}^{3} p_{2}+\mathrm{i} \frac{1084}{5625} 3^{2 / 3} \sqrt{5} q_{1}^{3} p_{1}+\mathrm{i} \frac{428}{9375} 3^{2 / 3} \sqrt{5} q_{1}^{2} q_{2} p_{2}-\frac{332}{375} 3^{2 / 3} q_{1}^{2} q_{2} p_{1} \\
& +\frac{724}{9375} 3^{2 / 3} q_{1} q_{2}^{2} p_{2}-\mathrm{i} \frac{15308}{46875} 3^{2 / 3} \sqrt{5} q_{1} q_{2}^{2} p_{1}+\mathrm{i} \frac{11572}{140625} 3^{2 / 3} \sqrt{5} q_{2}^{3} p_{2}+\frac{23116}{14062} 3^{2 / 3} q_{2}^{3} p_{1}, \\
& \mathscr{H}_{0,2,2}^{(1)}(\mu, q, p)=\frac{14}{625} 3^{2 / 3} q_{1}^{2} p_{2}^{2}+\mathrm{i} \frac{12}{625} 3^{2 / 3} \sqrt{5} q_{1}^{2} p_{1} p_{2}-\frac{638}{3125} 3^{2 / 3} q_{1}^{2} p_{1}^{2}+\mathrm{i} \frac{12}{625} 3^{2 / 3} \sqrt{5} q_{1} q_{2} p_{2}^{2}+\frac{7832}{9375} 3^{2 / 3} q_{1} q_{2} p_{1} p_{2} \\
& +\mathrm{i} \frac{196}{625} 3^{2 / 3} \sqrt{5} q_{1} q_{2} p_{1}^{2}-\frac{638}{3125} 3^{2 / 3} q_{2}^{2} p_{2}^{2}+\mathrm{i} \frac{196}{625} 3^{2 / 3} \sqrt{5} q_{2}^{2} p_{1} p_{2}-\frac{4946}{15625} 3^{2 / 3} q_{2}^{2} p_{1}^{2}, \\
& \mathscr{H}_{0,1,3}^{(1)}(\mu, q, p)=\frac{476}{1125} 3^{2 / 3} q_{1} p_{2}^{3}+\mathrm{i} \frac{428}{9375} 3^{2 / 3} \sqrt{5} q_{1} p_{1} p_{2}^{2}+\frac{724}{9375} 3^{2 / 3} q_{1} p_{1}^{2} p_{2}+\mathrm{i} \frac{11572}{140625} 3^{2 / 3} \sqrt{5} q_{1} p_{1}^{3} \\
& +\mathrm{i} \frac{1084}{5625} 3^{2 / 3} \sqrt{5} q_{2} p_{2}^{3}-\frac{332}{375} 3^{2 / 3} q_{2} p_{1} p_{2}^{2}-\mathrm{i} \frac{15308}{46875} 3^{2 / 3} \sqrt{5} q_{2} p_{1}^{2} p_{2}+\frac{23116}{140625} 3^{2 / 3} q_{2} p_{1}^{3}, \\
& \mathscr{H}_{0,0,4}^{(1)}(\mu, q, p)=-\frac{4043}{5625} 3^{2 / 3} p_{2}^{4}-\mathrm{i} \frac{3916}{28125} 3^{2 / 3} \sqrt{5} p_{1} p_{2}^{3}+\frac{1474}{3125} 3^{2 / 3} p_{1}^{2} p_{2}^{2}+\mathrm{i} \frac{748}{140625} 3^{2 / 3} \sqrt{5} p_{1}^{3} p_{2}+\frac{4453}{140625} 3^{2 / 3} p_{1}^{4} .
\end{aligned}
$$

Thus, as for $s=3$, we can state the homological equations, now for $\ell=1$, taking $M=2, N=0 ; M=0, N=2$; and for $\ell=0$, taking $M=4, N=0 ; M=3, N=1 ; M=1, N=3 ; M=0, N=4$. As before, form the linear systems of type (D.3) that follow from those equations, the component $G_{4}^{+}$of the generating functions are derived at once. Actually,

$$
G_{4}^{+}=G_{1,2,0}^{+}+G_{1,0,2}^{+}+G_{0,4,0}^{+}+G_{0,3,1}^{+}+G_{0,1,3}^{+}+G_{0,0,4}^{+},
$$


with,

$$
\begin{aligned}
G_{1,2,0}^{+}= & \mathrm{i} \frac{11}{50} 3^{2 / 3} \sqrt{5} \mu q_{1}^{2}-\frac{81}{50} 3^{2 / 3} \mu q_{1} q_{2}-\mathrm{i} \frac{71}{100} 3^{2 / 3} \sqrt{5} \mu q_{2}^{2}, \\
G_{1,0,2}^{+}= & -\mathrm{i} \frac{11}{50} 3^{2 / 3} \sqrt{5} \mu p_{2}^{2}+\frac{81}{50} 3^{2 / 3} \mu p_{1} p_{2}+\mathrm{i} \frac{71}{100} 3^{2 / 3} \sqrt{5} \mu p_{1}^{2}, \\
G_{0,4,0}^{+}= & \mathrm{i} \frac{4043}{37500} 3^{2 / 3} \sqrt{5} q_{1}^{4}-\frac{3209}{7500} 3^{2 / 3} q_{1}^{3} q_{2}-\mathrm{i} \frac{65823}{250000} 3^{2 / 3} \sqrt{5} q_{1}^{2} q_{2}^{2}+\frac{598391}{1500000} 3^{2 / 3} q_{1} q_{2}^{3}+\mathrm{i} \frac{1652677}{30000000} 3^{2 / 3} \sqrt{5} q_{2}^{4}, \\
G_{0,3,1}^{+}= & -\mathrm{i} \frac{238}{1875} 3^{2 / 3} \sqrt{5} q_{1}^{3} p_{2}+\frac{37}{375} 3^{2 / 3} q_{1}^{3} p_{1}+\frac{1999}{3125} 3^{2 / 3} q_{1}^{2} q_{2} p_{2}+\mathrm{i} \frac{2539}{15625} 3^{2 / 3} \sqrt{5} q_{1}^{2} q_{2} p_{1} \\
& +\mathrm{i} \frac{1127}{3125} 3^{2 / 3} \sqrt{5} q_{1} q_{2}^{2} p_{2}-\frac{13637}{31250} 3^{2 / 3} q_{1} q_{2}^{2} p_{1}-\frac{39143}{93750} 3^{2 / 3} q_{2}^{3} p_{2}-\mathrm{i} \frac{12884}{234375} 3^{2 / 3} \sqrt{5} q_{2}^{3} p_{1}, \\
G_{0,1,3}^{+}= & \mathrm{i} \frac{238}{1875} 3^{2 / 3} \sqrt{5} q_{1} p_{2}^{3}-\frac{1999}{3125} 3^{2 / 3} q_{1} p_{1} p_{2}^{2}-\mathrm{i} \frac{1127}{3125} 3^{2 / 3} \sqrt{5} q_{1} p_{1}^{2} p_{2}+\frac{39143}{93750} 3^{2 / 3} q_{1} p_{1}^{3} \\
& -\frac{37}{375} 3^{2 / 3} q_{2} p_{2}^{3}-\mathrm{i} \frac{2539}{15625} 3^{2 / 3} \sqrt{5} q_{2} p_{1} p_{2}^{2}+\frac{13637}{31250} 3^{2 / 3} q_{2} p_{1}^{2} p_{2}+\mathrm{i} \frac{12884}{234375} 3^{2 / 3} \sqrt{5} q_{2} p_{1}^{3}, \\
G_{0,0,4}^{+}= & -\mathrm{i} \frac{4043}{37500} 3^{2 / 3} \sqrt{5} p_{2}^{4}+\frac{3209}{7500} 3^{2 / 3} p_{1} p_{2}^{3}+\mathrm{i} \frac{65823}{250000} 3^{2 / 3} \sqrt{5} p_{1}^{2} p_{2}^{2}-\frac{598391}{1500000} 3^{2 / 3} p_{1}^{3} p_{2}-\mathrm{i} \frac{1652677}{30000000} 3^{2 / 3} \sqrt{5} p_{1}^{4} \triangleright{ }^{\circ}
\end{aligned}
$$

Analysis of the case $\Omega=0(M=N)$. Let $F \in \mathbb{E}_{\ell M M}$; it is readily seen that its expansion can be re-arranged in the following way

$$
\begin{aligned}
F=\sum_{i=0}^{M} \sum_{j=0}^{M} f_{i j} q_{1}^{i} q_{2}^{M-i} p_{1}^{M-j} p_{2}^{j}= & \sum_{i=0}^{M}\left(q_{1} p_{2}\right)^{i} \sum_{j=0}^{M-i} f_{M-j, i+j}\left(q_{1} p_{1}\right)^{M-i-j}\left(q_{2} p_{2}\right)^{j} \\
& +\sum_{i=1}^{M}\left(q_{2} p_{1}\right)^{i} \sum_{j=0}^{M-i} f_{M-i-j, j}\left(q_{1} p_{1}\right)^{M-i-j}\left(q_{2} p_{2}\right)^{j}
\end{aligned}
$$

(the same Remark AppendixD.2 applies here). The polynomials,

$$
\tilde{\xi}_{1}=q_{1} p_{2}, \quad \tilde{\xi}_{2}=q_{2} p_{1}, \quad \tilde{\xi}_{3}=\frac{\mathrm{i}}{2}\left(q_{1} p_{1}+q_{2} p_{2}\right), \quad \tilde{\xi}_{4}=\frac{1}{2}\left(q_{1} p_{1}-q_{2} p_{2}\right) .
$$

form a Hilbert basis of $\mathbb{C}^{\mathcal{S}}[[\nu, q, p]]^{\mathbb{S}^{1}}$, the formal power series in $\mathbb{C}^{\mathcal{S}}[[\nu, \alpha, \beta]]$ that are invariant under the $\mathbb{S}^{1}$ action generated by the flow of the Hamiltonian vector field $X_{\tilde{\xi}_{3}}$ (see [13], chap. I and IV). It turns out that,

$$
q_{1} p_{2}=\tilde{\xi}_{1}, \quad q_{2} p_{1}=\tilde{\xi}_{2}, \quad q_{1} p_{1}=-\mathrm{i} \tilde{\xi}_{3}+\tilde{\xi}_{4}, \quad q_{2} p_{2}=-\mathrm{i} \tilde{\xi}_{3}-\tilde{\xi}_{4},
$$

so substitution of these products into (D.7) leads to

$$
F=\sum_{i=0}^{M} \sum_{j=0}^{M} f_{i j} q_{1}^{i} q_{2}^{M-i} p_{1}^{M-j} p_{2}^{j}=\sum_{m=0}^{M} \tilde{\xi}_{1}^{m} \sum_{n=0}^{M-m} \widehat{f}_{m n} \tilde{\xi}_{3}^{M-m-n} \tilde{\xi}_{4}^{n}+\sum_{m=1}^{M} \tilde{\xi}_{2}^{m} \sum_{n=0}^{M-m} \tilde{f}_{m n} \tilde{\xi}_{3}^{M-m-n} \tilde{\xi}_{4}^{n}
$$

with,

$$
\begin{aligned}
& \widehat{f}_{m n}=(-\mathrm{i})^{M-m-n} \sum_{k=0}^{M-m} C(M-m-k, k, n) f_{M-k, m+k}, \quad \text { for } 0 \leq n \leq M-m, 0 \leq m \leq M, \\
& \widetilde{f}_{m n}=(-\mathrm{i})^{M-m-n} \sum_{k=0}^{M-m} C(M-m-k, k, n) f_{M-m-k, k}, \quad \text { for } 0 \leq n \leq M-m, 1 \leq m \leq M,
\end{aligned}
$$

being the coefficients

$$
C(r, k, n):=\sum_{j=\max (0, n-r)}^{\min (n, k)}(-1)^{j}\left(\begin{array}{c}
r \\
n-j
\end{array}\right)\left(\begin{array}{l}
k \\
j
\end{array}\right), \quad \text { for } 0 \leq r, k, n \leq M-m, 0 \leq m \leq M,
$$

where we use the notation introduced in the analysis of the previous case, now with $N=M$; i.e., as $\ell$ and $M$ are assumed fixed, then $f_{i j}=f_{\ell, i, M-i, M-j, j}$, for $0 \leq i \leq M, 0 \leq j \leq M$; $\widehat{f}_{m n}=\widehat{f}_{\ell, m, M-m, M-n, n}$, for $0 \leq n \leq M-m, 0 \leq m \leq M$; and $\widetilde{f}_{m n}=\widetilde{f}_{\ell, m, M-m, M-n, n}$, for $0 \leq n \leq M-m, 1 \leq m \leq M$. 


\begin{tabular}{c|cccc|c}
$\left\{\tilde{\xi}_{i}, \tilde{\xi}_{j}\right\}$ & $\tilde{\xi}_{1}$ & $\tilde{\xi}_{2}$ & $\tilde{\xi}_{3}$ & $\tilde{\xi}_{4}$ & $\tilde{\xi}_{j}$ \\
\hline$\tilde{\xi}_{1}$ & 0 & $-2 \tilde{\xi}_{4}$ & 0 & $\tilde{\xi}_{1}$ & \\
$\tilde{\xi}_{2}$ & $2 \tilde{\xi}_{4}$ & 0 & 0 & $-2 \tilde{\xi}_{2}$ & \\
$\tilde{\xi}_{3}$ & 0 & 0 & 0 & 0 & \\
$\tilde{\xi}_{4}$ & $-\tilde{\xi}_{1}$ & $\tilde{\xi}_{2}$ & 0 & 0 & \\
\cline { 1 - 1 }$\tilde{\xi}_{i}$ & & & &
\end{tabular}

Table D.1: Structure matrix for the Poisson bracket $\left\{\tilde{\xi}_{i}, \tilde{\xi}_{j}\right\}, i, j=1,2,3,4$.

Remark AppendixD.4. If we hold $m$ fixed, $0 \leq m \leq M$, and define

$$
\widehat{f}_{n}^{(m)}:=\widehat{f}_{m n}, \quad f_{k}^{(m)}:=f_{M-k, m+k}, \quad \widetilde{f}_{n}^{(m)}:=\widetilde{f}_{m n}, \quad \stackrel{\circ}{f}_{k}^{(m)}:=f_{M-. m-k, k}, \quad A_{n, k}^{(m)}:=C(M-m-k, k, n),
$$

for $0 \leq n, k \leq M-m$, then equations (D.10), (D.11) can be written in matrix form as,

$$
\widehat{f}_{n}^{(m)}=A_{n k}^{(m)} f_{k}^{(m)}, \quad \widetilde{f}_{n}^{(m)}=A_{n k}^{(m)} \stackrel{\circ}{f}_{k}^{(m)},
$$

respectively; and it is clear that they correspond to the change of components of $F$ as the basis changes from the canonical basis of $\mathbb{E}_{\ell M M}$ to the basis generated by $\tilde{\xi}_{1}, \tilde{\xi}_{2}, \tilde{\xi}_{3}$ and $\tilde{\xi}_{4}$ in (D.8).

Lemma 2. If $F \in \mathbb{E}_{\ell M M}^{\mathcal{S}}$, the components $\widehat{f}_{m n}(0 \leq n \leq M-m, 0 \leq m \leq M)$ and $\widetilde{f}_{m n}(0 \leq n \leq M-m, 1 \leq m \leq M)$ in (D.10) and (D.11) are real.

PROOF. It is enough to check that $\mathcal{S}\left(\tilde{\xi}_{i}\right)=\tilde{\xi}_{i}, i=1,2,3,4$, i.e., that the basis (D.8) satisfies the $\mathcal{S}$-symmetries.

To solve the homological equations (D.2) in the basis generated by (D.8), we first see that the quadratic part of the Hamiltonian, $Z_{2}$, is given by

$$
Z_{2}\left(\mu, \tilde{\xi}_{1}, \tilde{\xi}_{2}, \tilde{\xi}_{3}, \tilde{\xi}_{4}\right)=\frac{25}{8} \mu+\frac{2 \sqrt{5}}{3} \tilde{\xi}_{3}+\tilde{\xi}_{2}
$$

next, taking into account that, for any $u=u\left(\tilde{\xi}_{1}, \tilde{\xi}_{2}, \tilde{\xi}_{3}, \tilde{\xi}_{4}\right), v=v\left(\tilde{\xi}_{1}, \tilde{\xi}_{2}, \tilde{\xi}_{3}, \tilde{\xi}_{4}\right)$, their Poisson bracket is computed using the formula

$$
\begin{aligned}
\{u, v\}\left(\tilde{\xi}_{1}, \tilde{\xi}_{2}, \tilde{\xi}_{3}, \tilde{\xi}_{4}\right) & =\sum_{i, j=1}^{4} \frac{\partial u}{\partial \tilde{\xi}_{i}}\left(\tilde{\xi}_{1}, \tilde{\xi}_{2}, \tilde{\xi}_{3}, \tilde{\xi}_{4}\right)\left\{\tilde{\xi}_{i}, \tilde{\xi}_{j}\right\} \frac{\partial v}{\partial \tilde{\xi}_{j}}\left(\tilde{\xi}_{1}, \tilde{\xi}_{2}, \tilde{\xi}_{3}, \tilde{\xi}_{4}\right) \\
& =\nabla u\left(\tilde{\xi}_{1}, \tilde{\xi}_{2}, \tilde{\xi}_{3}, \tilde{\xi}_{4}\right)^{\top} S \nabla v\left(\tilde{\xi}_{1}, \tilde{\xi}_{2}, \tilde{\xi}_{3}, \tilde{\xi}_{4}\right),
\end{aligned}
$$

where the brackets $\left\{\tilde{\xi}_{i}, \tilde{\xi}_{j}\right\}$ are given in Table D.1. Therefore $S$ is the matrix with components $S_{i j}=\left\{\tilde{\xi}_{i}, \tilde{\xi}_{j}\right\}$, for $i, j=$ $1,2,3,4$. Now the relations

$$
L_{Z_{2}} \tilde{\xi}_{1}=\left\{\tilde{\xi}_{1}, Z_{2}\right\}=-2 \tilde{\xi}_{4}, \quad L_{Z_{2}} \tilde{\xi}_{2}=\left\{\tilde{\xi}_{2}, Z_{2}\right\}=0, \quad L_{Z_{2}} \tilde{\xi}_{3}=\left\{\tilde{\xi}_{3}, Z_{2}\right\}=0, \quad L_{Z_{2}} \tilde{\xi}_{4}=\left\{\tilde{\xi}_{4}, Z_{2}\right\}=\tilde{\xi}_{2},
$$

follow at once. Moreover,

$$
\begin{aligned}
L_{Z_{2}}\left(\tilde{\xi}_{1}^{m} \tilde{\xi}_{3}^{M-m-n} \tilde{\xi}_{4}^{n}\right) & =-2 m \tilde{\xi}_{1}^{m-1} \tilde{\xi}_{3}^{M-m-n} \tilde{\xi}_{4}^{n+1}+n \tilde{\xi}_{1}^{m-1} \tilde{\xi}_{3}^{M-m-n} \tilde{\xi}_{4}^{n-1} \tilde{\xi}_{1} \tilde{\xi}_{2} \\
& =-2 m \tilde{\xi}_{1}^{m-1} \tilde{\xi}_{3}^{M-m-n} \tilde{\xi}_{4}^{n+1}-n \tilde{\xi}_{1}^{m-1} \tilde{\xi}_{3}^{M-m-n} \tilde{\xi}_{4}^{n-1}\left(\tilde{\xi}_{3}^{2}+\tilde{\xi}_{4}^{2}\right) \\
& =-(2 m+n) \tilde{\xi}_{1}^{m-1} \tilde{\xi}_{3}^{M-m-n} \tilde{\xi}_{4}^{n+1}-n \tilde{\xi}_{1}^{m-1} \tilde{\xi}_{3}^{M-m-n+2} \tilde{\xi}_{4}^{n-1},
\end{aligned}
$$

for $m \geq 1$, and where we have used that

$$
\tilde{\xi}_{3}^{2}+\tilde{\xi}_{4}^{2}=-\tilde{\xi}_{1} \tilde{\xi}_{2}
$$

Analogously, for $n \geq 1$,

$$
L_{Z_{2}}\left(\tilde{\xi}_{2}^{m} \tilde{\xi}_{3}^{M-m-n} \tilde{\xi}_{4}^{n}\right)=n \tilde{\xi}_{2}^{m+1} \tilde{\xi}_{3}^{M-m-n} \tilde{\xi}_{4}^{n-1} .
$$

So we seek for polynomials $G, Z \in \mathbb{E}_{\ell M M}$, that fulfill the homological Eq. (D.2); with

$$
G=\sum_{m=1}^{M} \tilde{\xi}_{1}^{m} \sum_{n=0}^{M-m} \widehat{g}_{m n} \tilde{\xi}_{3}^{M-m-n} \tilde{\xi}_{4}^{n}+\sum_{m=0}^{M} \tilde{\xi}_{2}^{m} \sum_{n=0}^{M-m} \widetilde{g}_{m n} \tilde{\xi}_{3}^{M-m-n} \tilde{\xi}_{4}^{n}
$$

and $Z$ holding the resonant terms that belong to a complementary space of Range $L_{Z_{2}}$. 
Remark AppendixD.5. We note that the sums in (D.16) are arranged differently that those in (D.9); this is suggested by the action of $L_{Z_{2}}$ on the monomials $\tilde{\xi}_{1}^{m} \tilde{\xi}_{3}^{M-m-n} \tilde{\xi}_{4}^{n}$ and $\tilde{\xi}_{2}^{m} \tilde{\xi}_{3}^{M-m-n} \tilde{\xi}_{4}^{n}$ shown in (D.13) and in (D.15) respectively. $\diamond$ Hence, assuming $M \geq 3$, the homological Eq. (D.2) in the current polynomial basis, $\tilde{\xi}_{1}, \tilde{\xi}_{2}, \tilde{\xi}_{3}$, $\tilde{\xi}_{4}$, looks as

$$
\begin{aligned}
-2 M \widehat{g}_{M, 0} \tilde{\xi}_{1}^{M-1} \tilde{\xi}_{4}- & (2 M-2) \widehat{g}_{M-1,0} \tilde{\xi}_{1}^{M-2} \tilde{\xi}_{3} \tilde{\xi}_{4}-(2 M-1) \widehat{g}_{M-1,1} \tilde{\xi}_{1}^{M-2} \tilde{\xi}_{4}^{2}-\widehat{g}_{M-1,1} \tilde{\xi}_{1}^{M-2} \tilde{\xi}_{3}^{2} \\
& -\sum_{m=0}^{M-3}\left[\widehat{g}_{m+1,1} \tilde{\xi}_{3}^{M-m}+\sum_{n=1}^{M-m-2}\left\{(2 m+n+1) \widehat{g}_{m+1, n-1}+(n+1) \widehat{g}_{m+1, n+1}\right\} \times \tilde{\xi}_{3}^{M-m-n} \tilde{\xi}_{4}^{n}\right. \\
& \left.+(M+m) \widehat{g}_{m+1, M-m-2} \tilde{\xi}_{3} \tilde{\xi}_{4}^{M-m-1}+(M+m+1) \widehat{g}_{m+1, M-m-1} \tilde{\xi}_{4}^{M-m}\right] \times \tilde{\xi}_{1}^{m} \\
& +\sum_{m=1}^{M} \tilde{\xi}_{2}^{m} \sum_{n=0}^{M-m}(n+1) \tilde{g}_{m-1, n+1} \tilde{\xi}_{3}^{M-m-n} \tilde{\xi}_{4}^{n}+Z \\
= & \widehat{f}_{M, 0} \tilde{\xi}_{1}^{M}+\widehat{f}_{M-1,0} \tilde{\xi}_{1}^{M-1} \tilde{\xi}_{3}+\widehat{f}_{M-1,1} \tilde{\xi}_{1}^{M-1} \tilde{\xi}_{4}+\widehat{f}_{M-2,0} \tilde{\xi}_{1}^{M-2} \tilde{\xi}_{3}^{2}+\widehat{f}_{M-2,1} \tilde{\xi}_{1}^{M-2} \tilde{\xi}_{3} \tilde{\xi}_{4} \\
& +\widehat{f}_{M-2,2} \tilde{\xi}_{1}^{M-2} \tilde{\xi}_{4}^{2}+\sum_{m=0}^{M-3}\left[\widehat{f}_{m, 0} \tilde{\xi}_{3}^{M-m}+\sum_{n=1}^{M-m-2} \widehat{f}_{m n} \tilde{\xi}_{3}^{M-m-n} \tilde{\xi}_{4}^{n}\right. \\
& \left.+\sum_{m=1}^{M} \tilde{\xi}_{2}^{m} \sum_{n=0}^{M-m} \tilde{f}_{m n} \tilde{\xi}_{3}^{M-m-n} \tilde{\xi}_{4}^{n}, \quad \widehat{f}_{m, M-m-1} \tilde{\xi}_{3} \tilde{\xi}_{4}^{M-m-1}+\widehat{f}_{m, M-m} \tilde{\xi}_{4}^{M-m}\right] \times \tilde{\xi}_{1}^{m}
\end{aligned}
$$

so comparison of coefficients leads, on the one hand, to the linear system,

$$
\begin{aligned}
& -2 M \widehat{g}_{M, 0}=\widehat{f}_{M-1,1}, \\
& -(2 M-2) \widehat{g}_{M-1,0}=\widehat{f}_{M-2,1}, \\
& -(2 M-1) \widehat{g}_{M-1,1}=\widehat{f}_{M-2,2} \text {, } \\
& \left.\begin{array}{rl}
-(2 m+n+1) \widehat{g}_{m+1, n-1}-(n+1) \widehat{g}_{m+1, n+1} & =\widehat{f}_{m n}, \quad 1 \leq n \leq M-m-2, \\
-(M+m) \widehat{g}_{m+1, M-m-2} & =\widehat{f}_{m, M-m-1}, \\
-(M+m+1) \widehat{g}_{m+1, M-m-1} & =\widehat{f}_{m, M-m},
\end{array}\right\}, 0 \leq m \leq M-3 \\
& (n+1) \widetilde{g}_{m-1, n+1}=\widetilde{f}_{m n}, \quad 0 \leq n \leq M-m, \quad 1 \leq m \leq M
\end{aligned}
$$

(in fact, the last Eq. (D.22), holds also for $M=1$ and $M=2$ ). For $0 \leq m \leq M \geq 3, M \geq 3$ fixed, the linear system (D.21) is determinate compatible (it is a lower triangular system with no zeros at the diagonal).

Therefore, for $M \geq 3$, the components $\widehat{g}_{m n}, \widetilde{g}_{m n}, 0 \leq n \leq M-m, 0 \leq m \leq M$, of the generating function (D.16) are obtained solving the Eqs. (D.18)-(D.22), except the terms $\widetilde{g}_{m, 0}$ (for $0 \leq m \leq M$ ) that, due to the action of $L_{Z_{2}}$ (see (D.15)), do not appear in $L_{Z_{2}} G$. Consequently, they are not determined by the homological equations (they are "responsible for the non-uniqueness of $G$ "), and can be set arbitrarily. As in [41], we fix them to 0 . Furthermore, if $F \in \mathbb{E}_{\ell M M}^{\mathcal{S}}$, the right hand side of the system (D.18)-(D.22) is real (Lemma 2). Besides, as the choice $\widetilde{g}_{m, 0}=0$ (for $0 \leq m \leq M$ ) preserves the $\mathcal{S}$-symmetries, it follows that $G \in \mathbb{E}_{\ell M M}^{\mathcal{S}}$.

Resonant terms. For every $M$ fixed, we see that the component $\widehat{g}_{M-1,1}$ is determined by the Eq. (D.20) and $\widehat{g}_{m+1,1}$ $(0 \leq m \leq M-3)$ appear on left hand side of the first equation of (D.21) for $n=2$, so these components are also determined once the system is solved. Therefore, the terms $-\widehat{g}_{M-1,1} \tilde{\xi}_{1}^{M-2} \tilde{\xi}_{3}^{2}$ and $-\widehat{g}_{m+1,1} \tilde{\xi}_{1}^{m} \tilde{\xi}_{3}^{M-m}$ (for $0 \leq m \leq M-3$ ) cannot be used to remove the corresponding terms $\widehat{f}_{M-2,0} \tilde{\xi}_{1}^{M-2} \tilde{\xi}_{3}^{2}$ and $\widehat{f}_{m, 0} \tilde{\xi}_{1}^{m} \tilde{\xi}_{3}^{M-m}$ that are present at the right hand side of (D.17). Thus, all these terms -together with $\widehat{f}_{M, 0} \tilde{\xi}_{1}^{M}, \widehat{f}_{M-1,1} \tilde{\xi}_{1}^{M-1} \tilde{\xi}_{3}^{1}-$ must be included (the former ones, with opposite sign and their corresponding values substituted) in $Z$, at the left hand side of (D.17), so

$$
Z=\sum_{m=0}^{M} \widehat{f}_{m, 0} \tilde{\xi}_{1}^{m} \tilde{\xi}_{3}^{M-m}+\sum_{m=0}^{M-2} \widehat{g}_{m+1,1} \tilde{\xi}_{1}^{m} \tilde{\xi}_{3}^{M-m}=\sum_{m=0}^{M} \widehat{Z}_{m} \tilde{\xi}_{1}^{m} \tilde{\xi}_{3}^{M-m}
$$

where the components $\widehat{g}_{m+1,1}$ are given by the solution of Eqs. (D.18)-(D.21). Since all the coefficients in $Z$ are real, and $\mathcal{S}\left(\tilde{\xi}_{i}\right)=\tilde{\xi}_{i}, i=1,2,3,4$, we see that $Z \in \mathbb{E}_{\ell M M}^{\mathcal{S}}$.

On the other hand, for $M=1$, the working space of $\mathcal{S}$-symmetric homogeneous polynomials is $\mathbb{E}_{\ell, 1,1}^{\mathcal{S}}$, with $\ell=$ $1, \ldots 3\lfloor\sigma / 2\rfloor-\sigma$, for $\sigma=4,5, \ldots$ Then, according to (D.9) and (D.16),

$$
F=\widehat{f}_{1,0} \tilde{\xi}_{1}+\widetilde{f}_{1,0} \tilde{\xi}_{2}+\widehat{f}_{0,0} \tilde{\xi}_{3}+\widehat{f}_{0,1} \tilde{\xi}_{4}, \quad G=\widehat{g}_{1,0} \tilde{\xi}_{1}+\widetilde{g}_{1,0} \tilde{\xi}_{2}+\widetilde{g}_{0,0} \tilde{\xi}_{3}+\widetilde{g}_{0,1} \tilde{\xi}_{4},
$$


and $L_{Z_{2}} G=\widetilde{g}_{0,1} \tilde{\xi}_{2}-2 \widehat{g}_{1,0} \tilde{\xi}_{4}$, so the homological Eq. (D.2) is solved taking

$$
\widetilde{g}_{0,1}=\widetilde{f}_{1,0}, \quad \widehat{g}_{1,0}=-\frac{\widehat{f}_{0,1}}{2} .
$$

The coefficient $\widetilde{g}_{0,0}$ is not determined but, as we pointed out before, it can be set to 0 , so we take $\widetilde{g}_{0,0}=0$ and then

$$
G=-\frac{\widehat{f}_{0,1}}{2} \tilde{\xi}_{1}+\widetilde{f}_{1,0} \tilde{\xi}_{4}, \quad Z=\widehat{f}_{1,0} \tilde{\xi}_{1}+\widehat{f}_{0,0} \tilde{\xi}_{3}
$$

and clearly, $F, G \in \mathbb{E}_{\ell, 1,1}^{\mathcal{S}}$. Finally, for $M=2$,

$$
F=\widehat{f}_{1,1} \tilde{\xi}_{1} \tilde{\xi}_{4}+\widetilde{f}_{2,0} \tilde{\xi}_{2}^{2}+\tilde{f}_{1,0} \tilde{\xi}_{2} \tilde{\xi}_{3}+\widetilde{f}_{1,1} \tilde{\xi}_{2} \tilde{\xi}_{4}+\widehat{f}_{0,1} \tilde{\xi}_{3} \tilde{\xi}_{4}+\widehat{f}_{0,2} \tilde{\xi}_{4}^{2}+\widehat{f}_{2,0} \tilde{\xi}_{1}^{2}+\widehat{f}_{1,0} \tilde{\xi}_{1} \tilde{\xi}_{3}+\widehat{f}_{0,0} \tilde{\xi}_{3}^{2},
$$

and,

$$
G=\widehat{g}_{1,0} \tilde{\xi}_{1} \tilde{\xi}_{3}+\widehat{g}_{1,1} \tilde{\xi}_{1} \tilde{\xi}_{4}+\widehat{g}_{2,0} \tilde{\xi}_{1}^{2}+\widetilde{g}_{0,0} \tilde{\xi}_{3}^{2}+\widetilde{g}_{0,1} \tilde{\xi}_{3} \tilde{\xi}_{4}+\widetilde{g}_{0,2} \tilde{\xi}_{4}^{2}+\widetilde{g}_{1,0} \tilde{\xi}_{2} \tilde{\xi}_{3}+\widetilde{g}_{1,1} \tilde{\xi}_{2} \tilde{\xi}_{4}+\widetilde{g}_{2,0} \tilde{\xi}_{2}^{2},
$$

so the homological Eq. (D.2) takes the form,

$$
\begin{aligned}
-4 \widehat{g}_{2,0} \tilde{\xi}_{1} \tilde{\xi}_{4}+\widetilde{g}_{1,1} \tilde{\xi}_{2}^{2}+\widetilde{g}_{0,1} \tilde{\xi}_{2} \tilde{\xi}_{3}+2 \widetilde{g}_{0,2} \tilde{\xi}_{2} \tilde{\xi}_{4}-2 \widehat{g}_{1,0} \tilde{\xi}_{3} \tilde{\xi}_{4}-\widehat{g}_{1,1} \tilde{\xi}_{3}^{2}-3 \widehat{g}_{1,1} \tilde{\xi}_{4}^{2}+Z \\
\quad=\widehat{f}_{1,1} \tilde{\xi}_{1} \tilde{\xi}_{4}+\widetilde{f}_{2,0} \tilde{\xi}_{2}^{2}+\widetilde{f}_{1,0} \tilde{\xi}_{2} \tilde{\xi}_{3}+\widetilde{f}_{1,1} \tilde{\xi}_{2} \tilde{\xi}_{4}+\widehat{f}_{0,1} \tilde{\xi}_{3} \tilde{\xi}_{4}+\widehat{f}_{0,2} \tilde{\xi}_{4}^{2}+\widehat{f}_{2,0} \tilde{\xi}_{1}^{2}+\widehat{f}_{1,0} \tilde{\xi}_{1} \tilde{\xi}_{3}+\widehat{f}_{0,0} \tilde{\xi}_{3}^{2}
\end{aligned}
$$

and fixes the coefficients

$$
\widehat{g}_{2,0}=-\frac{\widehat{f}_{1,1}}{4}, \quad \widehat{g}_{1,0}=-\frac{\widehat{f}_{0,1}}{2}, \quad \widehat{g}_{1,1}=-\frac{\widehat{f}_{0,2}}{3}, \quad \widetilde{g}_{1,1}=\widetilde{f}_{2,0}, \quad \widetilde{g}_{0,1}=\widetilde{f}_{1,0}, \quad \widetilde{g}_{0,2}=\frac{\widetilde{f}_{1,1}}{2}
$$

of $G$. Again there the undetermined coefficients, and yet again they are set to 0 , i.e., $\widetilde{g}_{0,0}=\widetilde{g}_{1,0}=\widetilde{g}_{2,0}=0$. This gives rise to

$$
G=-\frac{\widehat{f_{0,1}}}{2} \tilde{\xi}_{1} \tilde{\xi}_{3}-\frac{\widehat{f}_{0,2}}{3} \tilde{\xi}_{1} \tilde{\xi}_{4}-\frac{\widehat{f}_{1,1}}{4} \tilde{\xi}_{1}^{2}+\widetilde{f}_{1,0} \tilde{\xi}_{3} \tilde{\xi}_{4}+\frac{\widetilde{f}_{1,1}}{2} \tilde{\xi}_{4}^{2}+\widetilde{f}_{2,0} \tilde{\xi}_{2} \tilde{\xi}_{4}
$$

whereas the components $\widehat{f}_{1,0}, \widehat{f}_{2,0}$ cannot be removed. Neither does $\widehat{f}_{0,0}$, since in this last case $\widehat{g}_{1,1}$ is first exacted by the homological Eq. (D.25) to $\widehat{g}_{1,1}=-\widehat{f}_{0,2} / 3$ (see (D.26)), so $-\widehat{g}_{1,1} \tilde{\xi}_{3}^{2}$ cannot be used to remove the term $\widehat{f}_{0,0} \tilde{\xi}_{3}^{2}$ on the right hand side of (D.25), and both them (the former with its opposite sign and its value substituted) must be present in $Z$. Consequently,

$$
Z=\widehat{f}_{0,0} \tilde{\xi}_{3}^{2}+\widehat{f}_{1,0} \tilde{\xi}_{1} \tilde{\xi}_{3}+\widehat{f}_{2,0} \tilde{\xi}_{1}^{2}+\widehat{g}_{1,1} \tilde{\xi}_{3}^{2}=\left(\widehat{f}_{0,0}-\frac{\widehat{f}_{0,2}}{3}\right) \tilde{\xi}_{3}^{2}+\widehat{f}_{1,0} \tilde{\xi}_{1} \tilde{\xi}_{3}+\widehat{f}_{2,0} \tilde{\xi}_{1}^{2} .
$$

Thus, we have shown that the normal form, $Z_{\sigma}$, depends only on $\mu, \tilde{\xi}_{1}=q_{1} p_{2}$ and $\tilde{\xi}_{3}=\frac{i}{2}\left(q_{1} p_{1}+q_{2} p_{2}\right)$. More precisely that, when $\sigma$ is even (and $\sigma \geq 4$ ) then $Z_{\sigma}(\mu, q, p)=\mathcal{Z}_{\sigma}\left(\mu, \tilde{\xi}_{1}, \tilde{\xi}_{3}\right)$, being $\mathcal{Z}_{\sigma}(u, v, w)$ a polynomial of standard degree $\sigma / 2$ with real coefficients. Furthermore, it is clear that the normal is given by (27). This closes the proof of statement (b) of Proposition 1.

Example 4 (Computation of $G_{4}^{0}$ and $Z_{4}$ ). We use (D.10) and (D.11) to express the components of $Z, G$ in (D.24), and $Z$, $G$ in (D.27) and (D.28) as linear combinations of the components of $\mathscr{H}_{1,1,1}^{(1)}$ and $\mathscr{H}_{0,2,2}^{(1)}$ with respect to the standard basis of $\mathbb{E}_{\ell M M}$. These components are shown in Table D.2, so

$$
\mathscr{H}_{\ell M M}^{(1)}=\sum_{m, n=0}^{M} \mathscr{H}_{\ell, m, M-m, M-n, n}^{(1)} q_{1}^{m} q_{2}^{M-m} p_{1}^{M-n} p_{2}^{n}
$$

corresponds to $\mathscr{H}_{1,1,1}^{(1)}$ in (D.5) for $\ell=1$ and $M=1$, whereas it matches $\mathscr{H}_{022}^{(1)}$ in (D.6) when $\ell=0$ and $M=2$. Therefore,

(i) for $M=1(\ell=1)$,

$$
\begin{aligned}
& \widehat{f}_{0,0}=-\mathrm{i}\left(f_{1,0}+f_{0,1}\right)=-\mathrm{i}\left(\mathscr{H}_{1,1,0,1,0}^{(1)}+\mathscr{H}_{1,0,1,0,1}^{(1)}\right)=\frac{28}{25} 3^{2 / 3} \sqrt{5} \\
& \widehat{f}_{0,1}=f_{1,0}-f_{0,1}=\mathscr{H}_{1,1,0,1,0}^{(1)}-\mathscr{H}_{1,0,1,0,1}^{(1)}=0 \\
& \widehat{f}_{1,0}=f_{1,1}=\mathscr{H}_{1,1,0,0,1}^{(1)}=\frac{6}{5} 3^{2 / 3} \\
& \widetilde{f}_{1,0}=f_{0,0}=\mathscr{H}_{1,0,1,1,0}^{(1)}=-\frac{51}{25} 3^{2 / 3},
\end{aligned}
$$




\begin{tabular}{cccccc}
\hline$\ell$ & $m_{1}$ & $m_{2}$ & $n_{1}$ & $n_{2}$ & $\mathscr{H}_{\ell m_{1} m_{2} n_{1} n_{2}}^{(1)}$ \\
\hline 1 & 1 & 0 & 0 & 1 & $\frac{6}{5} 3^{2 / 3}$ \\
1 & 1 & 0 & 1 & 0 & $\mathrm{i} \frac{14}{25} 3^{2 / 3} \sqrt{5}$ \\
1 & 0 & 1 & 0 & 1 & $\mathrm{i} \frac{14}{25} 3^{2 / 3} \sqrt{5}$ \\
1 & 0 & 1 & 1 & 0 & $-\frac{51}{25} 3^{2 / 3}$ \\
\hline 0 & 2 & 0 & 0 & 2 & $\frac{14}{625} 3^{2 / 3}$ \\
0 & 2 & 0 & 1 & 1 & $\mathrm{i} \frac{12}{625} 3^{2 / 3} \sqrt{5}$ \\
0 & 2 & 0 & 2 & 0 & $-\frac{638}{3125} 3^{2 / 3}$ \\
0 & 1 & 1 & 0 & 2 & $\mathrm{i} \frac{12}{625} 3^{2 / 3} \sqrt{5}$ \\
0 & 1 & 1 & 1 & 1 & $\frac{7832}{9375} 3^{2 / 3}$ \\
0 & 1 & 1 & 2 & 0 & $\mathrm{i} \frac{196}{625} 3^{2 / 3} \sqrt{5}$ \\
0 & 0 & 2 & 0 & 2 & $-\frac{638}{3125} 3^{2 / 3}$ \\
0 & 0 & 2 & 1 & 1 & $\mathrm{i} \frac{196}{625} 3^{2 / 3} \sqrt{5}$ \\
0 & 0 & 2 & 2 & 0 & $-\frac{4946}{15625} 3^{2 / 3}$ \\
\hline
\end{tabular}

Table D.2: Coefficients of homogeneous polynomials (of adapted degree $2 \ell+2 M=4) \mathscr{H}_{1,1,1}^{(1)}$ in (D.5) and $\mathscr{H}_{0,2,2}^{(1)}$ in (D.6), where $\mathscr{H}_{\ell M M}^{(1)}$ is given by the sum (D.29)

(ii) for $M=2(\ell=0)$,

$$
\begin{aligned}
& \widehat{f}_{0,0}=-\left(f_{2,0}+f_{1,1}+f_{0,2}\right)=-\left(\mathscr{H}_{0,2,0,2,0}^{(1)}+\mathscr{H}_{0,1,1,1,1}^{(1)}+\mathscr{H}_{0,0,2,0,2}^{(1)}\right)=-\frac{4004}{9375} 3^{2 / 3}, \\
& \widehat{f}_{1,0}=-\mathrm{i}\left(f_{2,1}+f_{1,2}\right)=-\mathrm{i}\left(\mathscr{H}_{0,2,0,1,1}^{(1)}+\mathscr{H}_{0,1,1,0,2}^{(1)}\right)=\frac{24}{625} 3^{2 / 3} \sqrt{5} \\
& \widehat{f}_{0,1}=-2 \mathrm{i}\left(f_{2,0}-f_{0,2}\right)=-2 \mathrm{i}\left(\mathscr{H}_{0,2,0,2,0}^{(1)}-\mathscr{H}_{0,0,2,0,2}^{(1)}\right)=0 \\
& \widehat{f}_{2,0}=f_{2,2}=\mathscr{H}_{0,2,0,0,2}^{(1)}=\frac{14}{625} 3^{2 / 3} \\
& \widehat{f}_{1,1}=f_{1,2}-f_{2,1}=\mathscr{H}_{0,2,0,1,1}^{(1)}-\mathscr{H}_{0,1,1,0,2}^{(1)}=0, \\
& \widehat{f}_{0,2}=f_{2,0}-f_{1,1}+f_{0,2}=\mathscr{H}_{0,2,0,2,0}^{(1)}-\mathscr{H}_{0,1,1,1,1}^{(1)}+\mathscr{H}_{0,0,2,0,2}^{(1)}=-\frac{2332}{1875} 3^{2 / 3} \\
& \widetilde{f}_{1,0}=-\mathrm{i}\left(f_{1,0}+f_{0,1}\right)=-\mathrm{i}\left(\mathscr{H}_{0,1,1,2,0}^{(1)}+\mathscr{H}_{0,0,2,1,1}^{(1)}\right)=\frac{392}{625} 3^{2 / 3} \sqrt{5} \\
& \widetilde{f}_{1,1}=f_{1,0}-f_{0,1}=\mathscr{H}_{0,1,1,2,0}^{(1)}-\mathscr{H}_{0,0,2,1,1}^{(1)}=0, \\
& \widetilde{f}_{2,0}=f_{0,0}=\mathscr{H}_{0,0,2,2,0}^{(1)}=-\frac{4946}{15625} 3^{2 / 3},
\end{aligned}
$$

(we have tagged the terms that cancel due to the reality conditions). Substitution of these coefficients in (D.24), (D.27) and (D.28) yields,

$$
\begin{aligned}
G_{1,1,1}^{0} & =\mathscr{H}_{1,0,1,1,0}^{(1)} \mu \tilde{\xi}_{4}=-\frac{51}{25} 3^{2 / 3} \mu \tilde{\xi}_{4}, \\
Z_{1,1,1}= & \mathscr{H}_{1,1,0,0,1}^{(1)} \mu \tilde{\xi}_{1}-\mathrm{i}\left(\mathscr{H}_{1,1,0,1,0}^{(1)}+\mathscr{H}_{1,0,1,0,1}^{(1)}\right) \mu \tilde{\xi}_{3}=\frac{6}{5} 3^{2 / 3} \mu \tilde{\xi}_{1}+\frac{28}{25} 3^{2 / 3} \sqrt{5} \mu \tilde{\xi}_{3}, \\
G_{0,2,2}^{0}= & -\frac{1}{3}\left(\mathscr{H}_{0,2,0,2,0}^{(1)}-\mathscr{H}_{0,1,1,1,1}^{(1)}+\mathscr{H}_{0,0,2,0,2}^{(1)}\right) \tilde{\xi}_{1} \tilde{\xi}_{4}-\mathrm{i}\left(\mathscr{H}_{0,1,1,2,0}^{(1)}+\mathscr{H}_{0,0,2,1,1}^{(1)}\right) \tilde{\xi}_{3} \tilde{\xi}_{4}+\mathscr{H}_{0,0,2,2,0}^{(1)} \tilde{\xi}_{2} \tilde{\xi}_{4} \\
= & \frac{2332}{5625} 3^{2 / 3} \tilde{\xi}_{1} \tilde{\xi}_{4}+\frac{392}{625} 3^{2 / 3} \sqrt{5} \tilde{\xi}_{3} \tilde{\xi}_{4}-\frac{4946}{15625} 3^{2 / 3} \tilde{\xi}_{2} \tilde{\xi}_{4},
\end{aligned}
$$




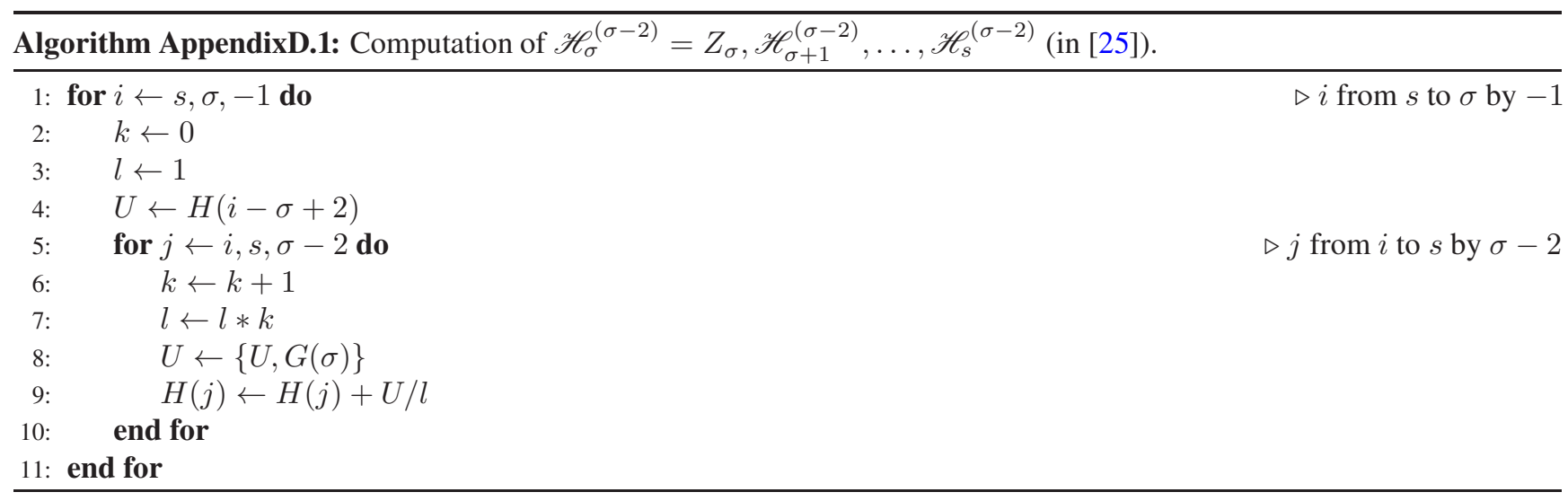

$$
\begin{aligned}
Z_{0,2,2} & =-\left(\frac{4}{3} \mathscr{H}_{0,2,0,2,0}^{(1)}+\frac{2}{3} \mathscr{H}_{0,1,1,1,1}^{(1)}+\frac{4}{3} \mathscr{H}_{0,0,2,0,2}^{(1)}\right) \tilde{\xi}_{3}^{2}-\mathrm{i}\left(\mathscr{H}_{0,2,0,1,1}^{(1)}+\mathscr{H}_{0,1,1,0,2}^{(1)}\right) \tilde{\xi}_{1} \tilde{\xi}_{3}+\mathscr{H}_{0,2,0,0,2}^{(1)} \tilde{\xi}_{1}^{2} \\
& =-\frac{352}{28125} 3^{2 / 3} \tilde{\xi}_{3}^{2}+\frac{24}{625} 3^{2 / 3} \sqrt{5} \tilde{\xi}_{1} \tilde{\xi}_{3}+\frac{14}{625} 3^{2 / 3} \tilde{\xi}_{1}^{2}
\end{aligned}
$$

where $\tilde{\xi}_{i}, i=1,2,3,4$ must be replaced by their definition in terms of $(q, p)$ in (D.8). Hence, $G_{4}^{0}=G_{1,1,1}^{0}+G_{0,2,2}^{0} \in \mathbb{E}_{4}^{0}$ and the complete generating function at degree $4, G_{4}$, is the sum $G_{4}=G_{4}^{+}+G_{4}^{0}$ (we recall that $G_{4}^{+} \in \mathbb{E}_{4}^{+}$was found in Example 3). Besides, the (complex) normal form up to (adapted) degree 4 results to be

$$
\begin{aligned}
Z^{(4)}=Z_{2}+Z_{2,0,0}+Z_{1,1,1}+Z_{0,2,2}= & \frac{25}{8} \mu+\frac{27}{32} 3^{2 / 3} \mu^{2}+\frac{2}{3} \sqrt{5} \tilde{\xi}_{3}+\tilde{\xi}_{2}+\frac{6}{5} 3^{2 / 3} \mu \tilde{\xi}_{1}+\frac{28}{25} 3^{2 / 3} \sqrt{5} \mu \tilde{\xi}_{3} \\
& -\frac{352}{28125} 3^{2 / 3} \tilde{\xi}_{3}^{2}+\frac{24}{625} 3^{2 / 3} \sqrt{5} \tilde{\xi}_{1} \tilde{\xi}_{3}+\frac{14}{625} 3^{2 / 3} \tilde{\xi}_{1}^{2},
\end{aligned}
$$

being $Z_{2,0,0}=\mathscr{H}_{2,0,0}^{(1)}=\frac{27}{32} 3^{2 / 3} \mu^{2}$. This corresponds to the trivial case $M=0$, see (D.4).

\section{Some remarks on the normal form}

Remark AppendixD.6 (On the complexification). We note that, since the Poisson brackets preserve the $\mathcal{S}$-symmetries (see Remark AppendixC.1 (iii)), and we have seen that the generating functions $G_{\sigma}, \sigma=3,4, \ldots$, are $\mathcal{S}$-invariants as long as the each of the corresponding $\mathscr{H}_{\sigma}^{(\sigma-3)}$ in the right hand side of (25) is; then it follows by induction that the successive transformed Hamiltonians, $\mathscr{H}^{(\sigma-2)}$, given by (26) will also be $\mathcal{S}$-invariants.

Remark AppendixD.7. In practice, there are several useful algorithms to perform the computations in an effective way. For example, once the homological Eq. (25) are solved and $G_{\sigma}$ is found, one can use Algorithm AppendixD.1 above (see [25] for the corresponding software implementation) to compute the terms of the transformed Hamiltonian, $\mathscr{H}^{(\sigma-2)}$, from the current (adapted) degree $\sigma$, up to the given degree $s$ to which the normal form is computed; more precisely, it outputs,

$$
\mathscr{H}_{\sigma}^{(\sigma-2)}=Z_{\sigma}, \mathscr{H}_{\sigma+1}^{(\sigma-2)}, \ldots, \mathscr{H}_{s}^{(\sigma-2)} .
$$

Remark AppendixD.8. The transformation generated by $G_{3}$ are given by,

$$
\begin{aligned}
& q_{i}^{(0)}=q_{i}^{(1)}+\left\{q_{i}^{(1)}, G_{3}\right\}+\frac{1}{2 !}\left\{\left\{q_{i}^{(1)}, G_{3}\right\}, G_{3}\right\}+\frac{1}{3 !}\left\{\left\{\left\{q_{i}^{(1)}, G_{3}\right\}, G_{3}\right\}, G_{3}\right\}+\ldots, \\
& p_{i}^{(0)}=p_{i}^{(1)}+\left\{p_{i}^{(1)}, G_{3}\right\}+\frac{1}{2 !}\left\{\left\{p_{i}^{(1)}, G_{3}\right\}, G_{3}\right\}+\frac{1}{3 !}\left\{\left\{\left\{p_{i}^{(1)}, G_{3}\right\}, G_{3}\right\}, G_{3}\right\}+\ldots,
\end{aligned}
$$

$i=1,2$; where $\left(q^{(0)}, p^{(0)}\right)$ are the original coordinates and $\left(q^{(1)}, p^{(1)}\right)$ are the new ones. This can be repeated to get explicitly the symplectic changes generated by $G_{4}, G_{5}, \ldots$ and so on, up to the required degree. Of course, a similar algorithm to Algorithm AppendixD.1 can be applied coordinatewise to mechanize the computations (again, see [25] for details).

Remark AppendixD.9. The normal form at 1:-1 non semisimple resonant equilibrium point in a family of Hamiltonian systems has been investigated in many papers, here we quote: [50], [55], [56]. The same resonance can take place in a family of periodic orbits (see [23], [40], [41], [42], [43]), and in a family of lower dimensional invariant tori (see [8]). Whatever the algorithm to perform the transformations is, the key point of the reduction to normal form lies in the solvability of the homological Eq. (25) (see the quoted references for an outlook of the different approaches). In the context of the periodic Hopf bifurcation, this is tackled in [41], Proposition 4.1, which we adapt here to the special case of a 3-degree of freedom (complexified) Hamiltonian (21), depending on the normal coordinates $q, p \in \mathbb{C}^{2}$, an "action" $\mu \in \mathbb{C}$ (originally, the real parameter of the family of Hamiltonians), but not explicitly on its conjugate angle. 
Of course, one might expand the Hamiltonian (21) with respect to the $(q, p)$ coordinates only, letting the coefficients depend on $\mu$. Proceeding in that way, the coefficients of the normal form terms and of the generating functions -given, at each step, by the solutions of (25)- would now be rational functions of the parameter $\mu$. Thus, the higher the degree, the more intricate the expressions. This results to be a very expensive way to compute the normal form. Hence, the approach we present here of expanding in the parameter (we recall that it is assumed to be a small quantity), seems fairly more suitable. $\diamond$

Remark AppendixD.10. In some situations, such as when we approximate the solutions of the original Hamiltonian system using the solutions of its normal form or when we look for the "optimal" degree $s$ up to what the normal form has to be computed to minimize the size of the remainder, we have to find explicitly the generating functions $G_{3}, G_{4}, \ldots$ At this regard, we stress that the above proof of Proposition 1 given in this appendix shows, not only the structure of the reduced Hamiltonian in this case in point, but also provides the formulas for its coefficients and the coefficients of the generating functions, up to any required degree.

Remark AppendixD.11. The Examples 1-4 that came up along the proof of Proposition 1, illustrate the practical implementation of the normalization process for the given Hamiltonian of the CP problem. In particular, the fourth order normal form, $Z^{(4)}$, of the Hamiltonian (21) (and the generating functions $G_{3}$ and $G_{4}$ ) follow from the Examples 3, and 4. We remark that the proof of Proposition 1 we give here is more complete and detailed than the one given in [41].

\section{References}

[1] G. B. Arfken, H. J. Weber, Mathematical Methods for Physicists, sixth ed., Elsevier Academic Press, San Diego, Cal., 2005. 23

[2] V. I. Arnold, V. V. Kozlov, A. I. Neĭshtadt, Dynamical Systems. III, vol. 3 of Encyclopaedia of Mathematical Sciences, Springer-Verlag, Berlin, 1988, translated from the Russian by A. Iacob. 10

[3] B. Bardin, On motions near the Lagrange equilibrium point $L_{4}$ in the case of Routh's critical mass ratio, Celest. Mech. Dyn. Astr. 82 (2) (2002) 163-177. 10

[4] E. Barrabés, J. Mondelo, M. Ollé, Numerical continuation of families of homoclinic connections of periodic orbits in the RTBP, Nonlinearity 22 (12) (2009) 2901-2918. 16

[5] E. Barrabés, M. Ollé, F. Borondo, D. Farrelly, J. M. Mondelo, Phase space structure of the hydrogen atom in a circularly polarized microwave field, Physica D: Nonlinear Phenomena 241 (4) (2012) 333-349. 2, 5, 18

[6] F. Benvenuto, G. Casati, D. L. Shepelyansky, Reply letter, Phys. Rev. Lett. 74 (1995) 3496. 1

[7] T. J. Bridges, J. E. Furter, Singularity theory and equivariant symplectic maps, vol. 1558 of Lecture Notes in Mathematics, Springer-Verlag, Berlin, 1993. 25

[8] H. W. Broer, H. Hanßmann, J. Hoo, The quasi-periodic Hamiltonian Hopf bifurcation, Nonlinearity 20 (2) (2007) 417460. $3,10,11,34$

[9] R. Broucke, Stability of periodic orbits in the Elliptic Restricted Three-body Problem, AIAA J. 7 (6) (1969) $1003-1009$. 15

[10] A. F. Brunello, T. Uzer, D. Farrelly, Hydrogen atom in circularly polarized microwaves: Chaotic ionization via core scattering, Phys. Rev. 55 (5) (1997) 3730-3745. 1, 4, 18

[11] A. D. Bruno, Instability in Hamilton's system and the distribution of asteroids, Mat. Sb. (N.S.) 83 (125) (1970) $273-312$. 10,11

[12] A. D. Bruno, Normalization of a Hamiltonian system near an invariant cycle or torus, Uspekhi Mat. Nauk 44 (2(266)) (1989) 49-78. 10, 11

[13] R. H. Cushman, L. M. Bates, Global Aspects of Classical Integrable Systems, Birkhäuser Verlag, Basel, 1997. 29

[14] R. H. Cushman, J. C. van der Meer, Orbiting dust under radiation pressure, in: Proc. of the XVth International Conference on Differential Geometric Methods in Theoretical Physics, H. B. Doebner and J. D. Hennig (ed.), Singapore: World Scientific, Germany, 1986, pp. 403-414. 1

[15] A. Deprit, Dynamics of orbiting dust under radiation pressure, in: The Big Bang and Georges Lemaitre, A. Berger (ed.), D. Reidel, Dordrecht, 1984, pp. 151-180. 1

[16] D. Farrelly, P. Bellomo, T. Uzer, Comment on "Chaotic autoionization of molecular Rydberg states", Phys. Rev. Lett. 74 (17) (1995) 3495. 1

[17] D. Farrelly, T. Uzer, Ionization mechanism of Rydberg atoms in a circularly polarized microwave field, Phys. Rev. Lett. 74 (10) (1995) 1720-1723. 2, 3 
[18] L. B. Fu, G. G. Xin, D. F. Ye, J. Li, Recollision dynamics and phase diagram for nonsequential double ionization with circularly polarized laser fields, Phys. Rev. Lett. 108 (10) (2012) 103601. 2

[19] P. Fu, T. J. Scholz, J. M. Hettema, T. F. Gallagher, Ionization of Rydberg atoms by a circularly polarized microwave field, Phys. Rev. Lett. 64 (5) (1995) 511-514. 1

[20] R. Gebarowski, J. J. Zakrzewski, Ionization of hydrogen atoms by circularly polarized microwaves, Phys. Rev. A 51 (1995) 1508-1519. 1

[21] A. Giorgilli, A. Delshams, E. Fontich, L. Galgani, C. Simó, Effective stability for a Hamiltonian system near an elliptic equilibrium point, with an application to the restricted three-body problem, J. Diff. Eq. 77 (1) (1989) 167-198. 22, 25

[22] J. A. Griffiths, D. Farrelly, Ionization of Rydberg atoms by circularly and elliptically polarized microwave fields, Phys. Rev. A 45 (5) (1992) 2678-2681. 2

[23] D. C. Heggie, Bifurcation at complex instability, Celest. Mech. 35 (4) (1985) 357-382. 11, 34

[24] C. Jaffé, D. Farrelly, T. Uzer, Transition state theory without time-reversal symmetry: Chaotic ionization of the hydrogen atom, Phys. Rev. Lett. 84 (4) (2000) 610-613. 2

[25] À. Jorba, A methodology for the numerical computation of normal forms, centre manifolds and first integrals of Hamiltonian systems, Experiment. Math. 8 (2) (1999) 155-195. 34

[26] À. Jorba, J. Villanueva, On the normal behaviour of partially elliptic lower-dimensional tori of Hamiltonian systems, Nonlinearity 10 (4) (1997) 783-822. 10, 11

[27] À. Jorba, M. Zou, A software package for the numerical integration of ODEs by means of high-order Taylor methods, Experiment. Math. 14 (1) (2005) 99-117. 3

[28] A. Kamor, F. Mauger, C. Chandre, T. Uzer, How key periodic orbits drive recollisions in a circularly polarized laser field, Phys. Rev. Lett. 110 (2013) 253002. 2

[29] A. Lahiri, M. Roy, The hamiltonian Hopf bifurcation: an elementary perturbative approach, Int. J. Non-Linear Mech. 36 (2001) 787-802. 2, 3, 10

[30] V. Lanchares, M. Iñarrea, J. P. Salas, Bifurcations in the hydrogen atom in the presence of a circularly polarized microwave field and a static magnetic field, Phys. Rev. A 56 (3) (1997) 1839-1843. 2

[31] A. J. Laub, K. Meyer, Canonical forms for symplectic and Hamiltonian matrices, Celest. Mech. 9 (1974) $213-238.7$

[32] L. M. Lerman, A. P. Markova, On stability at the Hamiltonian Hopf bifurcation, Regul. Chaotic Dyn. 14 (1) (2009) 148-162. 10, 11

[33] F. Mauger, C. Chandre, T. Uzer, Recollisions and correlated double ionization with circularly polarized light, Phys. Rev. Lett. 105 (2010) 083002. 2

[34] K. R. Meyer, G. R. Hall, D. Offin, Introduction to Hamiltonian Dynamical Systems and the N-Body Problem, vol. 90 of Applied Mathematical Sciences, 2nd ed., Springer, New York, 2009. 5, 7, 9, 10

[35] K. R. Meyer, J. F. Palacián, P. Yanguas, Stability of a Hamiltonian system in a limiting case, Regul. Chaotic Dyn. 17 (1) (2012) 24-35. 10

[36] K. R. Meyer, D. S. Schmidt, Periodic orbits near $L_{4}$ for mass ratios near the critical mass ratio of Routh, Celest. Mech. 4 (1971) 99-109. 10

[37] M. Nauenberg, Canonical Kepler map, Europhys. Lett. 13 (1990) 611-616. 1

[38] M. Nauenberg, Comment on "Ionization of Rydberg atoms by a circularly polarized microwave field", Phys. Rev. Lett. 64 (1990) 27-31. 1

[39] M. Ollé, To and fro motion for the hydrogen atom in a circularly polarized microwave field, Commun. Nonlinear Sci. Numer. Simulat. 54 (2017) 286-301. 2, 18

[40] M. Ollé, J. R. Pacha, J. Villanueva, Dynamics close to a non semi-simple 1:-1 resonant periodic orbit, Discr. Cont. Dyn. Syst. Ser. B 5 (3) (2005) 799-816. 13, 34

[41] M. Ollé, J. R. Pacha, J. Villanueva, Quantitative estimates on the normal form around a non-semi-simple 1:-1 resonant periodic orbit, Nonlinearity 18 (3) (2005) 1141-1172. 7, 9, 25, 31, 34, 35

[42] M. Ollé, J. R. Pacha, J. Villanueva, Kolmogorov-Arnold-Moser aspects of the periodic Hamiltonian Hopf bifurcation, Nonlinearity 21 (8) (2008) 1759-1811. 34 
[43] J. R. Pacha, On the Quasi-Periodic Hamiltonian Andronov-Hopf Bifurcation, Ph.D. thesis, Facultat de Matemàtiques, Universitat Politècnica de Catalunya (October 2002). 34

[44] A. Peregrine-Smew, D. Farrelly, T. Uzer, Comment on "Circular Rydberg orbits in circularly polarized microwave radiation", Phys. Rev. A 51 (5) (1995) 4293-4294. 1

[45] M. J. Rakovic, S. I. Chu, Approximate dynamical symmetry of hydrogen atoms in circularly polarized microwave fields, Phys. Rev. A 50 (6) (1994) 5077-5080. 2

[46] M. J. Rakovic, S. I. Chu, New integrable systems: hydrogen atom in external fields, Phys. D 81 (3) (1995) 207-316. 2

[47] D. L. Richardson, Analytic construction of periodic orbits about the collinear points, Celest. Mech. 22 (3) (1980) 241253. 22

[48] D. L. Richardson, A note on a Lagrangian formulation for motion about the collinear points, Celest. Mech. 22 (3) (1980) 231-236. 22

[49] K. Rzazewski, B. B. Piraux, Circular Rydberg orbits in circularly polarized microwave radiation, Phys. Rev. A 47 (1993) 1612-1614. 1

[50] D. S. Schmidt, Versal normal form of the Hamiltonian function of the restricted problem of three bodies near $L_{4}$, J. Comput. Appl. Math. 52 (1-3) (1994) 155-176. 5, 9, 34

[51] C. L. Siegel, J. K. Moser, Lectures on Celestial Mechanics, Classics in Mathematics, Springer-Verlag, Berlin, 1995.5

[52] C. Simó, Estabilitat de sistemes hamiltonians, Mem. Reial Acad. Ciènc. Arts Barcelona 48 (7) (1989) 305-336. 25

[53] A. G. Sokolskiľ, On the stability of an autonomous Hamiltonian system with two degrees of freedom in the case of equal frequencies, Prikl. Mat. Meh. 38 (1974) 791-799. 7, 9, 10

[54] A. G. Sokolskiı̌, Proof of the stability of Lagrangian solutions for a critical mass ratio, Pis'ma Astron. Zh. 4 (1978) 184-152. 9, 10, 11

[55] J. C. van der Meer, Nonsemisimple $1: 1$ resonance at an equilibrium, Celest. Mech. 27 (2) (1982) 131-149. 34

[56] J. C. van der Meer, The Hamiltonian Hopf Bifurcation, vol. 1160 of Lecture Notes in Mathematics, Springer-Verlag, Berlin, 1985. 5, 9, 10, 34

[57] J. Williamson, On the algebraic problem concerning the normal forms of linear dynamical systems, Amer. J. Math. 58 (1) (1936) 141-163. 7

[58] J. Williamson, On the normal forms of linear canonical transformations in dynamics, Amer. J. Math. 59 (3) (1937) 599-617. 7

[59] J. Williamson, The exponential representation of canonical matrices, Amer. J. Math. 61 (1939) 897-911. 7

[60] J. Zakrzewski, D. Delande, J. C. Gay, Ionization of hoghly excited hydrogen atoms by a circularly polarized microwave field, Phys. Rev. A 47 (4) (1993) R2468-R2471. 1 